Supporting Information

\title{
Synthesis of Ocotillol-type Ginsenosides
}

Renzeng Shen, ${ }^{\mathrm{a}}$ Xin Cao, ${ }^{\mathrm{a}, *}$ Stephane Laval, ${ }^{\mathrm{a}}$ Jiansong Sun, ${ }^{\mathrm{b}}$ and Biao Yu ${ }^{\mathrm{a}, *}$

a State Key Laboratory of Bio-organic and Natural Products Chemistry, Shanghai Institute of Organic Chemistry, Chinese Academy of Sciences, 345 Lingling Road, Shanghai 200032, China.

${ }^{b}$ National Research Center for Carbohydrate Synthesis, Jiangxi Normal University, 437 West Beijing Road, Nanchang, 330027, China.

X-Ray Crystallographic structures of compounds $3 \mathrm{~b}, 6 \mathrm{a}$ and $\mathrm{S1}$ S2

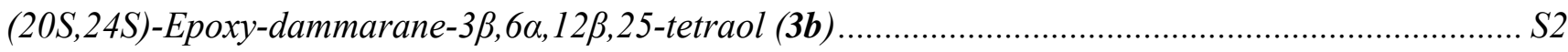

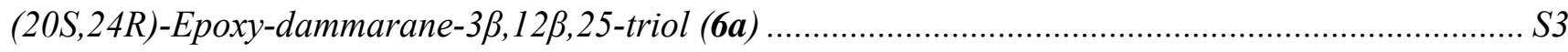

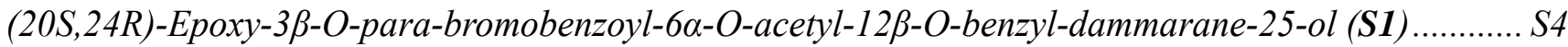

Comparison of the ${ }^{13} \mathrm{C}$ NMR data of the chemically synthesized ocotillol-type ginsenosides with those reported in the literature for the synthetic or natural products.................................................................. S5

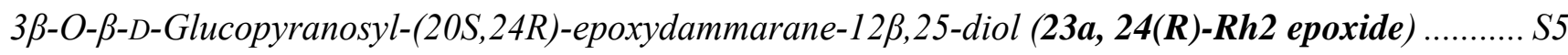

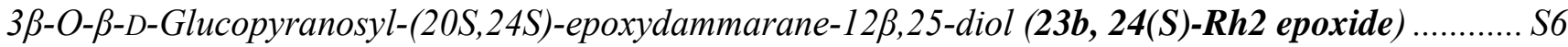

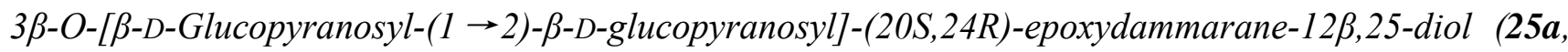

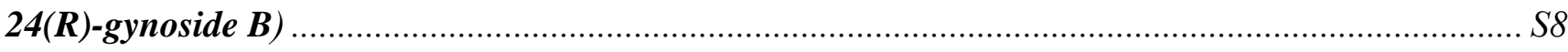

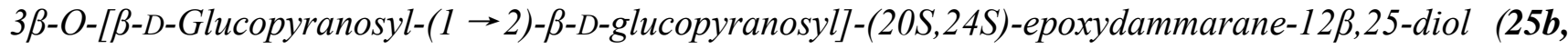

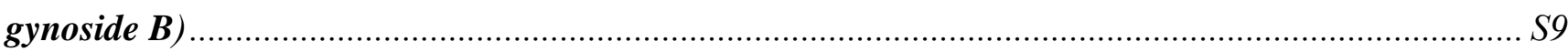

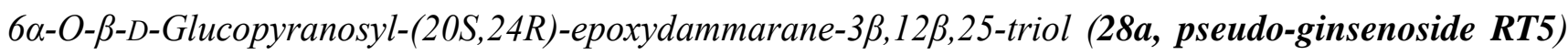

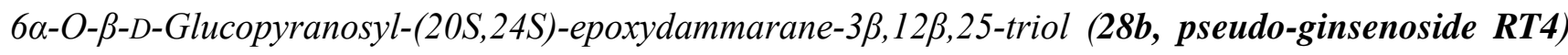

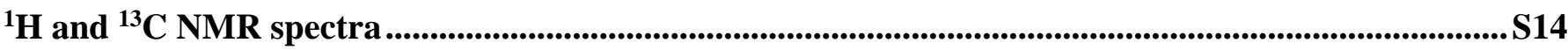


X-Ray Crystallographic structures of compounds 3b, 6a and S1

(20S,24S)-Epoxy-dammarane-3 $\beta, 6 \alpha, 12 \beta, 25$-tetraol (3b)
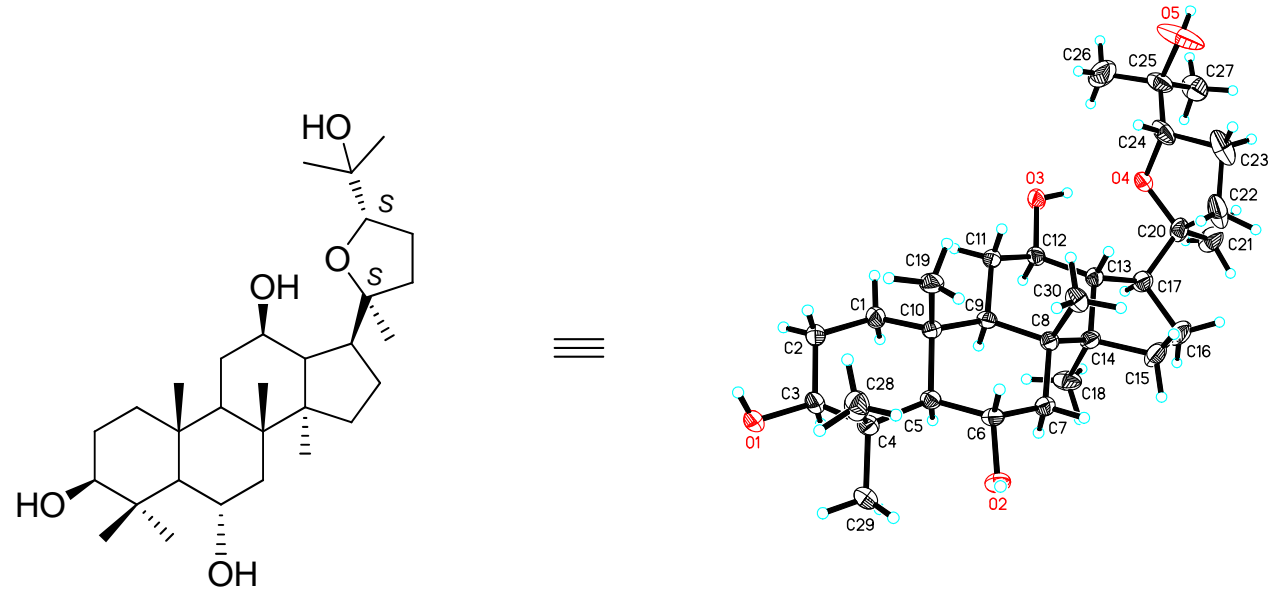

Figure 1. ORTEP figure of $\mathbf{3 b}$ with thermal ellipsoids shown at 30\% probability

Table 1 . Hydrogen bonds for $\mathbf{3 b}\left[\AA\right.$ and $\left.{ }^{\circ}\right]$

\begin{tabular}{|c|c|c|c|c|}
\hline D-H...A & d(D-H) & d(H...A & d(D...A) & $<(\mathrm{DHA})$ \\
\hline $\mathrm{O}(5)-\mathrm{H}(5) \ldots \mathrm{O}(1)^{\mathrm{i}}$ & 0.82 & 1.91 & $2.685(3)$ & 157.8 \\
\hline $\mathrm{O}(3)-\mathrm{H}(3) \ldots \mathrm{O}(4)$ & 0.82 & 1.87 & $2.669(3)$ & 163.4 \\
\hline $\mathrm{O}(2)-\mathrm{H}(2) \ldots \mathrm{O}(3)^{\mathrm{ii}}$ & 0.82 & 1.94 & $2.751(3)$ & 170.0 \\
\hline $\mathrm{O}(1)-\mathrm{H}(1) \ldots \mathrm{O}(2)^{\mathrm{iii}}$ & 0.82 & 2.04 & $2.850(3)$ & 170.4 \\
\hline $\mathrm{O}(1)-\mathrm{H}(1) \ldots \mathrm{O}(2)^{\mathrm{iii}}$ & 0.82 & 2.04 & $2.850(3)$ & 170.4 \\
\hline $\mathrm{O}(2)-\mathrm{H}(2) \ldots \mathrm{O}(3)^{\mathrm{ii}}$ & 0.82 & 1.94 & $2.751(3)$ & 170.0 \\
\hline $\mathrm{O}(3)-\mathrm{H}(3) \ldots \mathrm{O}(4)$ & 0.82 & 1.87 & $2.669(3)$ & 163.4 \\
\hline $\mathrm{O}(5)-\mathrm{H}(5) \ldots \mathrm{O}(1)^{\mathrm{i}}$ & 0.82 & 1.91 & $2.685(3)$ & 157.8 \\
\hline
\end{tabular}

(i) $\mathrm{x}, \mathrm{y}, \mathrm{z}-1$; (ii) $-\mathrm{x}+2, \mathrm{y}-1 / 2,-\mathrm{z}+1 / 2$; (iii) $\mathrm{x}+1 / 2,-\mathrm{y}+3 / 2,-\mathrm{z}+1$. 

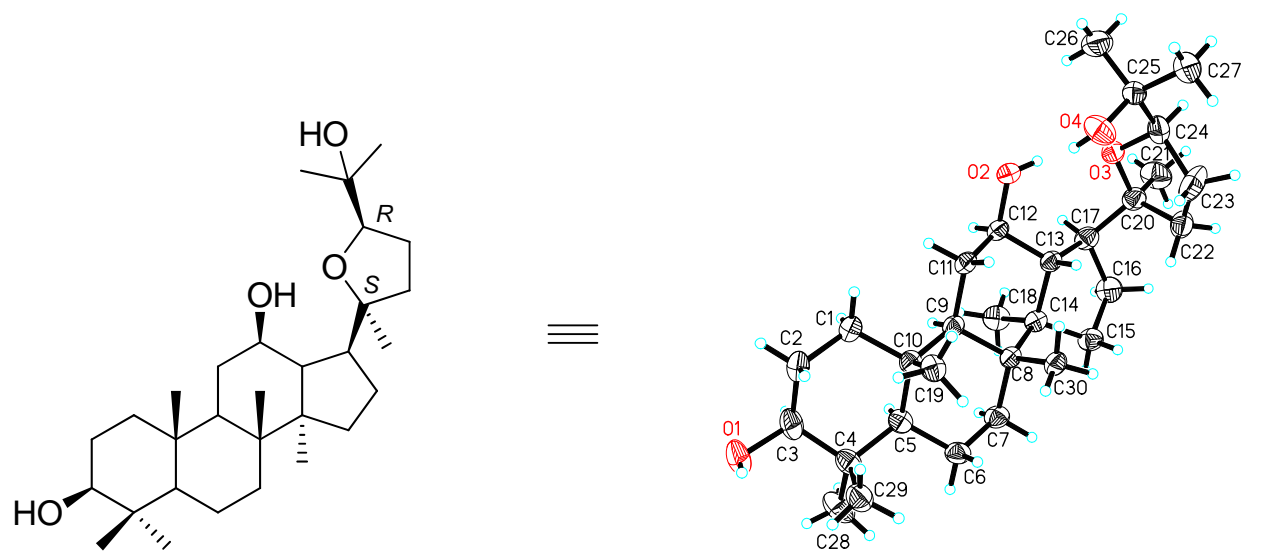

Figure 2. ORTEP figure of $\mathbf{6 a}$ with thermal ellipsoids shown at 30\% probability

Table 2. Hydrogen bonds for $\mathbf{6 a}\left[\AA\right.$ and $\left.{ }^{\circ}\right]$

\begin{tabular}{|c|c|c|c|c|}
\hline D-H...A & d(D-H) & $\mathrm{d}(\mathrm{H} . . . \mathrm{A})$ & $\mathrm{d}(\mathrm{D} \ldots \mathrm{A})$ & $<(\mathrm{DHA})$ \\
\hline $\mathrm{O}(1)-\mathrm{H}(1) \ldots \mathrm{O}(4)^{\mathrm{i}}$ & 0.82 & 2.10 & 2.907 & 167.7 \\
\hline $\mathrm{O}(2)-\mathrm{H}(2) \ldots \mathrm{O}(3)$ & 0.82 & 1.89 & 2.6757 & 160.5 \\
\hline $\mathrm{O}(4)-\mathrm{H}(4) \ldots \mathrm{O}(2)$ & 0.82 & 2.14 & 2.945 & 167.7 \\
\hline
\end{tabular}

(i) $\mathrm{x}-1 / 2,-y-1 / 2,-z+2$. 

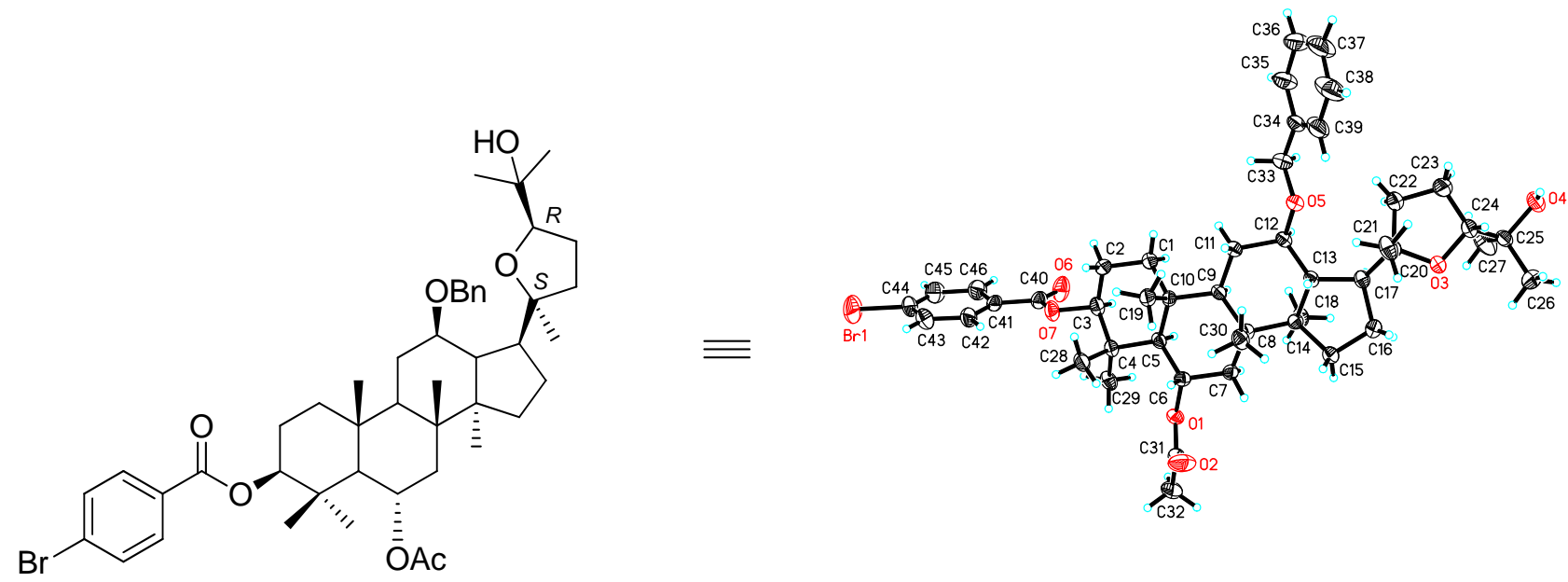

Figure 3. ORTEP figure of S1 with thermal ellipsoids shown at 30\% probability

Table 3. Hydrogen bonds for $\mathbf{S 1}\left[\AA\right.$ and ${ }^{\circ}$ ]

\begin{tabular}{|c|c|c|c|c|}
\hline D-H...A & $\mathrm{d}(\mathrm{D}-\mathrm{H})$ & $\mathrm{d}(\mathrm{H} \ldots \mathrm{A})$ & $\mathrm{d}(\mathrm{D} \ldots \mathrm{A})$ & $<(\mathrm{DHA})$ \\
\hline $\mathrm{C}(22)-\mathrm{H}(22) \ldots \mathrm{O}(5)$ & 0.97 & 2.52 & 3.061 & 115.4 \\
\hline
\end{tabular}


Comparison of the ${ }^{13} \mathrm{C}$ NMR data of the chemically synthesized ocotillol-type ginsenosides with those reported in the literature for the synthetic or natural products.

The references used for the comparison of the ${ }^{13} \mathrm{C}$ NMR are as follows:

(1) Bi, Y.; Wang, T.; Meng, Q.; Zhang, J.; Wang, L.; Li, Q.; Zhao, F.; Sun, H. Rec. Nat. Prod. 2012, 6, 242-254.

(2) 'Standard NMR Spectrum of Ginsenosides’, ed. by P. Li, Chemical Industry Press, 2012.

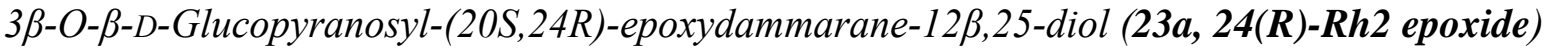

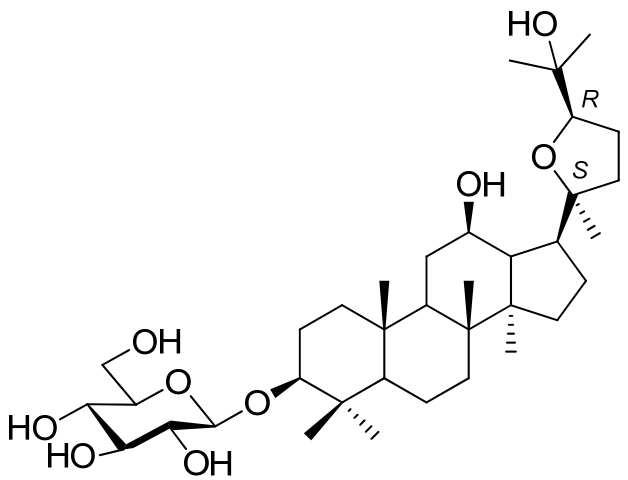

\begin{tabular}{|l|l|l|}
\hline C & Synthetic 24(R)-Rh2 epoxide (23a) & Reported data [ref (1)] \\
\hline 1 & 39.2 & 39.2 \\
\hline 2 & 26.6 & 26.7 \\
\hline 3 & 88.8 & 88.7 \\
\hline 4 & 40.0 & 40.0 \\
\hline 5 & 56.4 & 56.4 \\
\hline 6 & 18.4 & 18.4 \\
\hline 7 & 35.1 & 35.1 \\
\hline 8 & 36.9 & 36.9 \\
\hline 9 & 49.5 & 50.0 \\
\hline 10 & 39.6 & 39.7 \\
\hline 11 & 32.3 & 32.4 \\
\hline 12 & 71.0 & 71.0 \\
\hline 13 & 48.3 & 48.3 \\
\hline 14 & 50.7 & 50.7 \\
\hline 15 & 31.6 & 31.6 \\
\hline 16 & 25.4 & 25.5 \\
\hline 17 & 52.1 & 52.1 \\
\hline
\end{tabular}




\begin{tabular}{|l|l|l|}
\hline 18 & 16.7 & 16.7 \\
\hline 19 & 16.5 & 16.5 \\
\hline 20 & 86.7 & 86.7 \\
\hline 21 & 27.0 & 27.2 \\
\hline 22 & 32.8 & 32.8 \\
\hline 23 & 28.7 or 28.1 & 28.1 \\
\hline 24 & 85.5 & 85.6 \\
\hline 25 & 70.4 & 70.3 \\
\hline 26 & 27.2 & 27.6 \\
\hline 27 & 26.91 & 26.9 \\
\hline 28 & 28.1 or 28.7 & 28.1 \\
\hline 29 & 15.5 & 15.5 \\
\hline 30 & 18.3 & 18.3 \\
\hline $1^{\prime}$ & 106.8 & 106.7 \\
\hline $2^{\prime}$ & 75.6 & 75.8 \\
\hline $3^{\prime}$ & 78.4 & 78.8 \\
\hline $4^{\prime}$ & 71.6 & 71.9 \\
\hline $5^{\prime}$ & 78.2 & 78.4 \\
\hline $6^{\prime}$ & 62.8 & 63.1 \\
\hline
\end{tabular}

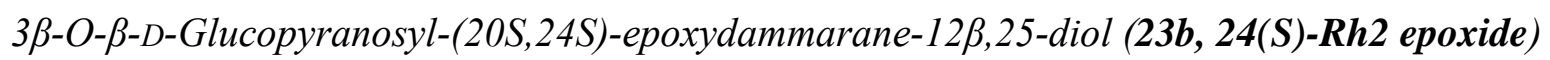

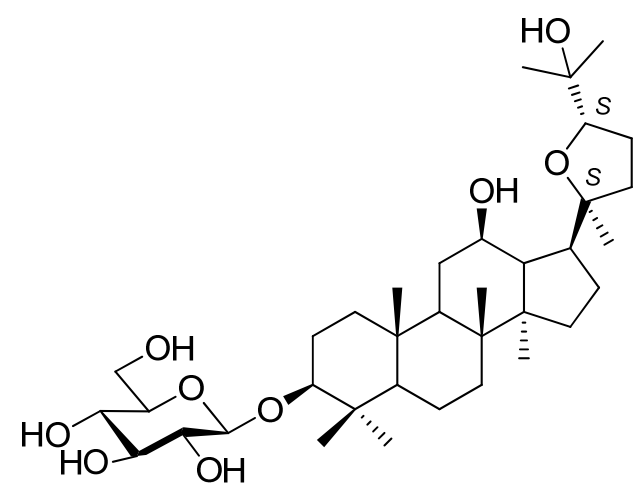

\begin{tabular}{|l|l|l|}
\hline C & Synthetic 24(S)-Rh2 epoxide (23b) & Reported data [ref (2)] \\
\hline 1 & 39.1 & 39.1 \\
\hline 2 & 26.6 & 26.6 \\
\hline 3 & 88.6 & 88.6 \\
\hline 4 & 39.8 & 39.8 \\
\hline 5 & 56.3 & 56.3 \\
\hline 6 & 18.3 & 18.3 \\
\hline 7 & 35.0 & 35.0 \\
\hline 8 & 36.8 & 36.8 \\
\hline
\end{tabular}




\begin{tabular}{|c|c|c|}
\hline 9 & 50.4 & 50.4 \\
\hline 10 & 39.5 & 39.5 \\
\hline 11 & 32.4 & 32.4 \\
\hline 12 & 70.6 & 70.6 \\
\hline 13 & 49.30 or 49.27 & 49.3 \\
\hline 14 & 52.1 & 52.1 \\
\hline 15 & 32.5 & 32.5 \\
\hline 16 & 25.6 & 25.6 \\
\hline 17 & 49.27 or 49.30 & 49.3 \\
\hline 18 & 15.4 & 15.4 \\
\hline 19 & 16.6 & 16.6 \\
\hline 20 & 86.9 & 86.9 \\
\hline 21 & 26.4 & 26.4 \\
\hline 22 & 32.0 & 32.0 \\
\hline 23 & 28.5 & 28.5 \\
\hline 24 & 88.2 & 88.2 \\
\hline 25 & 69.8 & 69.8 \\
\hline 26 & 26.8 & 26.8 \\
\hline 27 & 28.8 & 28.8 \\
\hline 28 & 27.9 & 27.9 \\
\hline 29 & 16.4 & 16.4 \\
\hline 30 & 17.9 & 17.9 \\
\hline $1^{\prime}$ & 106.8 & 106.8 \\
\hline $2^{\prime}$ & 75.6 & 75.6 \\
\hline 3 & 78.6 & 78.6 \\
\hline 4 ' & 71.7 & 71.7 \\
\hline 5 ' & 78.2 & 78.2 \\
\hline $6^{\prime}$ & 62.9 & 62.9 \\
\hline
\end{tabular}




\section{4(R)-gynoside B)}

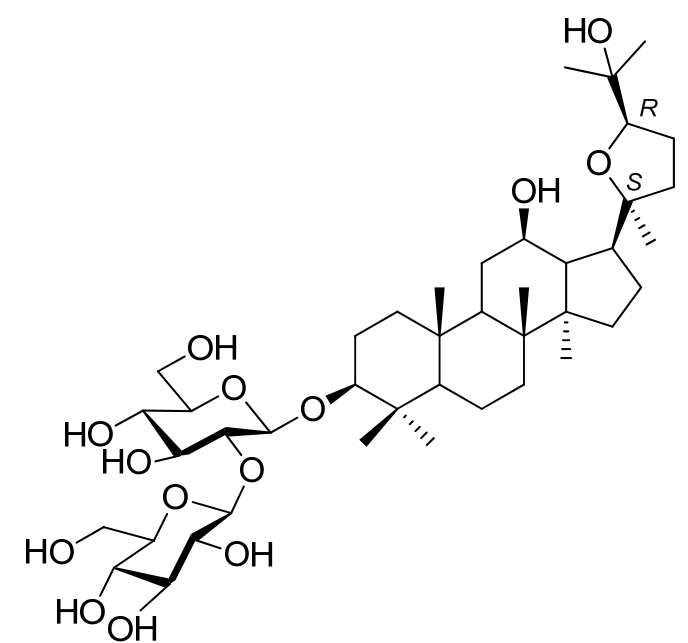

\begin{tabular}{|c|c|c|}
\hline $\mathrm{C}$ & Synthetic 24(R)-gynoside B (25a) & Reported data [ref (2)] \\
\hline 1 & 39.3 & 39.3 \\
\hline 2 & 26.8 & 26.8 \\
\hline 3 & 88.9 & 88.9 \\
\hline 4 & 39.8 & 39.8 \\
\hline 5 & 56.5 & 56.5 \\
\hline 6 & 18.5 & 18.5 \\
\hline 7 & 35.2 & 35.2 \\
\hline 8 & 40.1 & 40.0 \\
\hline 9 & 50.8 & 50.8 \\
\hline 10 & 37.0 & 37.0 \\
\hline 11 & 32.5 & 32.5 \\
\hline 12 & 71.2 & 71.2 \\
\hline 13 & 48.5 & 48.4 \\
\hline 14 & 52.3 & 52.2 \\
\hline 15 & 32.9 & 32.9 \\
\hline 16 & 25.6 & 25.5 \\
\hline 17 & 49.8 & 49.8 \\
\hline 18 & 15.6 & 15.6 \\
\hline 19 & 16.6 & 16.6 \\
\hline 20 & 86.8 & 86.8 \\
\hline 21 & 27.3 & 27.3 \\
\hline 22 & 31.7 & 31.7 \\
\hline 23 & 28.9 & 28.8 \\
\hline 24 & 85.7 & 85.7 \\
\hline
\end{tabular}




\begin{tabular}{|l|l|l|}
\hline 25 & 70.4 & 70.4 \\
\hline 26 & 27.0 & 27.0 \\
\hline 27 & 27.8 & 27.7 \\
\hline 28 & 28.1 & 28.1 \\
\hline 29 & 16.6 & 16.6 \\
\hline 30 & 18.4 & 18.4 \\
\hline 3-Glc 1' & 105.2 & 105.2 \\
\hline 2' & 83.6 & 83.6 \\
\hline 3' & 78.0 & 78.0 \\
\hline 4' & 71.70 & 71.6 \\
\hline 5' & 78.2 & 78.2 \\
\hline 6' & 62.9 & 62.9 \\
\hline 2'-Glc 1'” & 106.2 & 106.2 \\
\hline 2'” & 77.3 & 77.2 \\
\hline 3’' & 78.4 & 78.4 \\
\hline 4'” & 71.75 & 71.7 \\
\hline 5’' & 78.3 & 78.3 \\
\hline 6’' & 62.8 & 62.8 \\
\hline
\end{tabular}

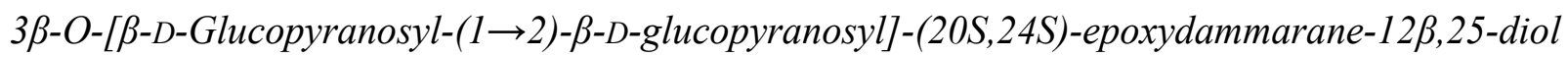

\section{gynoside B)}

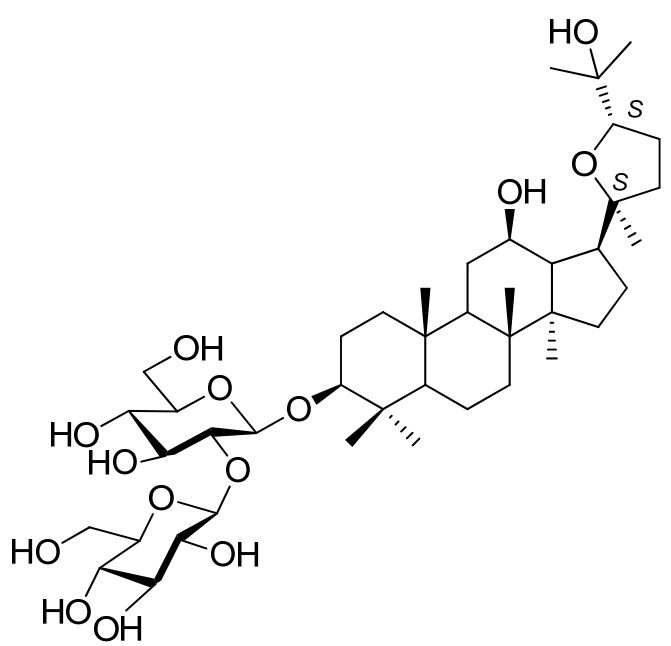

\begin{tabular}{|l|l|l|}
\hline C & Synthetic gynoside B (25b) & Reported data [ref (2)] \\
\hline 1 & 39.4 & 39.4 \\
\hline 2 & 26.9 & 26.9 \\
\hline 3 & 89.0 & 89.1 \\
\hline 4 & 39.8 & 39.8 \\
\hline 5 & 56.6 & 56.6 \\
\hline
\end{tabular}




\begin{tabular}{|c|c|c|}
\hline 6 & 18.6 & 18.6 \\
\hline 7 & 35.3 & 35.3 \\
\hline 8 & 40.1 & 40.1 \\
\hline 9 & 50.7 & 50.7 \\
\hline 10 & 37.1 & 37.1 \\
\hline 11 & 32.7 & 32.7 \\
\hline 12 & 70.9 & 70.9 \\
\hline 13 & 49.63 or 49.61 & 49.6 \\
\hline 14 & 52.4 & 52.4 \\
\hline 15 & 32.8 & 32.8 \\
\hline 16 & 25.9 & 25.9 \\
\hline 17 & 49.61 or 49.63 & 49.6 \\
\hline 18 & 15.7 & 15.7 \\
\hline 19 & 16.7 & 16.7 \\
\hline 20 & 87.2 & 87.2 \\
\hline 21 & 26.7 & 26.7 \\
\hline 22 & 32.3 & 32.3 \\
\hline 23 & 28.8 & 28.8 \\
\hline 24 & 88.5 & 88.5 \\
\hline 25 & 70.1 & 70.1 \\
\hline 26 & 27.1 & 27.1 \\
\hline 27 & 29.1 & 29.1 \\
\hline 28 & 28.2 & 28.2 \\
\hline 29 & 16.7 & 16.7 \\
\hline 30 & 18.2 & 18.2 \\
\hline 3-Glc 1' & 105.2 & 105.2 \\
\hline $2^{\prime}$ & 83.6 & 83.6 \\
\hline 3 & 78.1 & 78.1 \\
\hline $4^{\prime}$ & 71.7 & 71.7 \\
\hline 5 & 78.2 & 78.2 \\
\hline $6^{\prime}$ & 63.0 & 63.0 \\
\hline 2'-Glc 1"' & 106.2 & 106.2 \\
\hline 2" & 77.3 & 77.2 \\
\hline $3 ”$ & 78.5 & 78.5 \\
\hline $4 "$ & 71.8 & 71.8 \\
\hline $5 ”$ & 78.4 & 78.3 \\
\hline $6 "$ & 62.8 & 62.9 \\
\hline
\end{tabular}




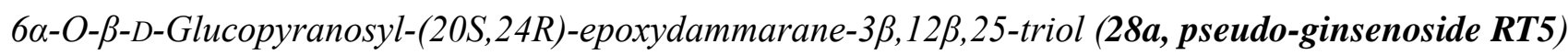

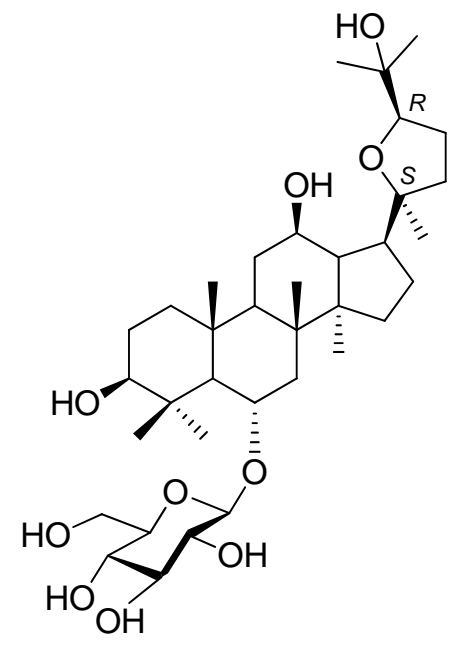

\begin{tabular}{|c|c|c|}
\hline $\mathrm{C}$ & Synthetic pseudo-ginsenoside RT5 (28a) & Reported data [ref (2)] \\
\hline 1 & 39.6 & 39.6 \\
\hline 2 & 27.9 & 27.9 \\
\hline 3 & 78.6 & 78.6 \\
\hline 4 & 40.4 & 40.4 \\
\hline 5 & 61.5 & 61.5 \\
\hline 6 & 80.1 & 80.1 \\
\hline 7 & 45.1 & 45.1 \\
\hline 8 & 40.0 & 41.0 \\
\hline 9 & 50.6 & 50.6 \\
\hline 10 & 39.5 & 39.5 \\
\hline 11 & 32.4 & 32.4 \\
\hline 12 & 71.2 & 71.2 \\
\hline 13 & 49.4 & 49.4 \\
\hline 14 & 52.2 & 52.2 \\
\hline 15 & 32.7 & 32.7 \\
\hline 16 & 25.5 & 25.4 \\
\hline 17 & 48.4 & 48.4 \\
\hline 18 & 17.1 & 17.1 \\
\hline 19 & 17.9 & 17.9 \\
\hline 20 & 86.7 & 86.7 \\
\hline 21 & 27.0 & 27.0 \\
\hline 22 & 31.7 & 31.7 \\
\hline 23 & 28.8 & 28.8 \\
\hline 24 & 85.6 & 85.6 \\
\hline 25 & 70.3 & 70.4 \\
\hline 26 & 27.2 & 27.1 \\
\hline
\end{tabular}




\begin{tabular}{|l|l|l|}
\hline 27 & 27.7 & 27.6 \\
\hline 28 & 31.7 & 31.7 \\
\hline 29 & 16.3 & 16.3 \\
\hline 30 & 18.0 & 18.0 \\
\hline $1^{\prime}$ & 106.0 & 106.0 \\
\hline $2^{\prime}$ & 75.5 & 75.5 \\
\hline $3^{\prime}$ & 79.7 & 79.7 \\
\hline $4^{\prime}$ & 71.9 & 71.9 \\
\hline $5^{\prime}$ & 78.2 & 78.2 \\
\hline $6^{\prime}$ & 63.1 & 63.1 \\
\hline
\end{tabular}

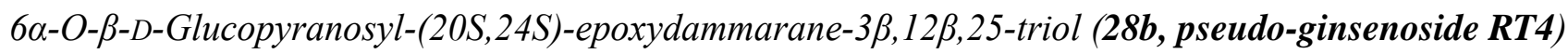

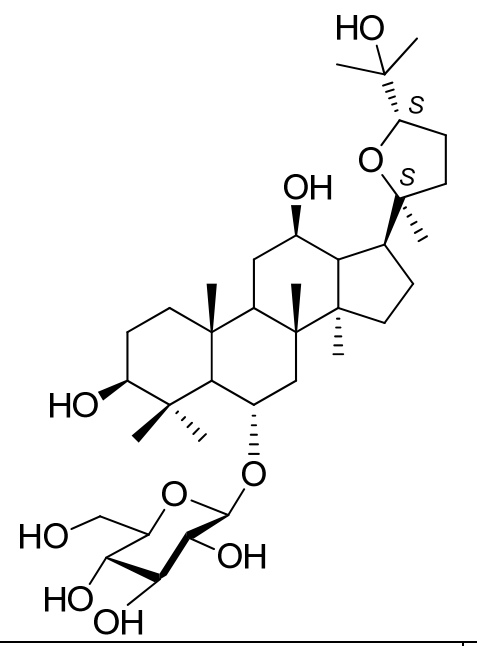

\begin{tabular}{|l|l|l|}
\hline C & Synthetic pseudo-ginsenoside RT4 (28b) & Reported data [ref (2)] \\
\hline 1 & 39.6 & 39.5 \\
\hline 2 & 28.0 & 28.0 \\
\hline 3 & 78.6 & 78.6 \\
\hline 4 & 40.4 & 40.4 \\
\hline 5 & 61.6 & 61.6 \\
\hline 6 & 80.2 & 80.2 \\
\hline 7 & 45.2 & 45.1 \\
\hline 8 & 41.1 & 41.1 \\
\hline 9 & 50.4 & 50.4 \\
\hline 10 & 39.7 & 39.7 \\
\hline 11 & 32.6 & 32.5 \\
\hline 12 & 70.9 & 70.9 \\
\hline 13 & 49.2 & 49.1 \\
\hline 14 & 52.3 & 52.2 \\
\hline 15 & 32.7 & 32.7 \\
\hline & & \\
\hline
\end{tabular}




\begin{tabular}{|l|l|l|}
\hline 16 & 25.8 & 25.8 \\
\hline 17 & 49.5 & 49.5 \\
\hline 18 & 17.2 & 17.2 \\
\hline 19 & 17.9 & 17.9 \\
\hline 20 & 87.1 & 87.1 \\
\hline 21 & 27.0 & 27.0 \\
\hline 22 & 32.3 & 32.2 \\
\hline 23 & 28.7 & 28.7 \\
\hline 24 & 88.5 & 88.5 \\
\hline 25 & 70.0 & 70.0 \\
\hline 26 & 26.6 & 26.6 \\
\hline 27 & 29.0 & 29.0 \\
\hline 28 & 31.7 & 31.7 \\
\hline 29 & 16.4 & 16.4 \\
\hline 30 & 17.8 & 17.8 \\
\hline $1^{\prime}$ & 106.1 & 106.1 \\
\hline $2^{\prime}$ & 75.5 & 75.5 \\
\hline $3^{\prime}$ & 79.7 & 79.7 \\
\hline $4^{\prime}$ & 71.9 & 71.9 \\
\hline $5^{\prime}$ & 78.3 & 78.3 \\
\hline $6^{\prime}$ & 63.1 & 63.1 \\
\hline & & \\
\hline & & \\
\hline
\end{tabular}




\section{${ }^{1} \mathrm{H}$ and ${ }^{13} \mathrm{C}$ NMR spectra}
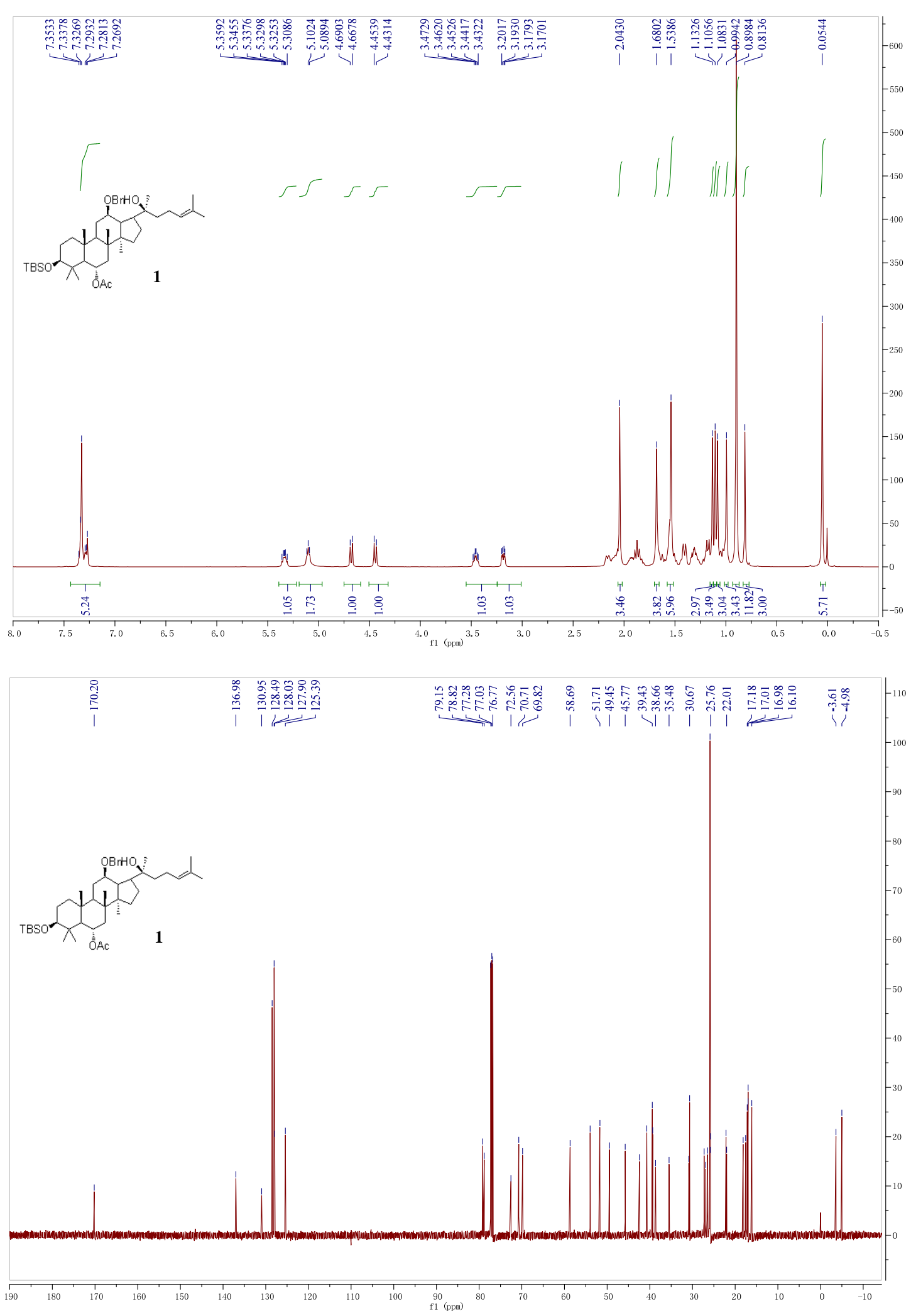

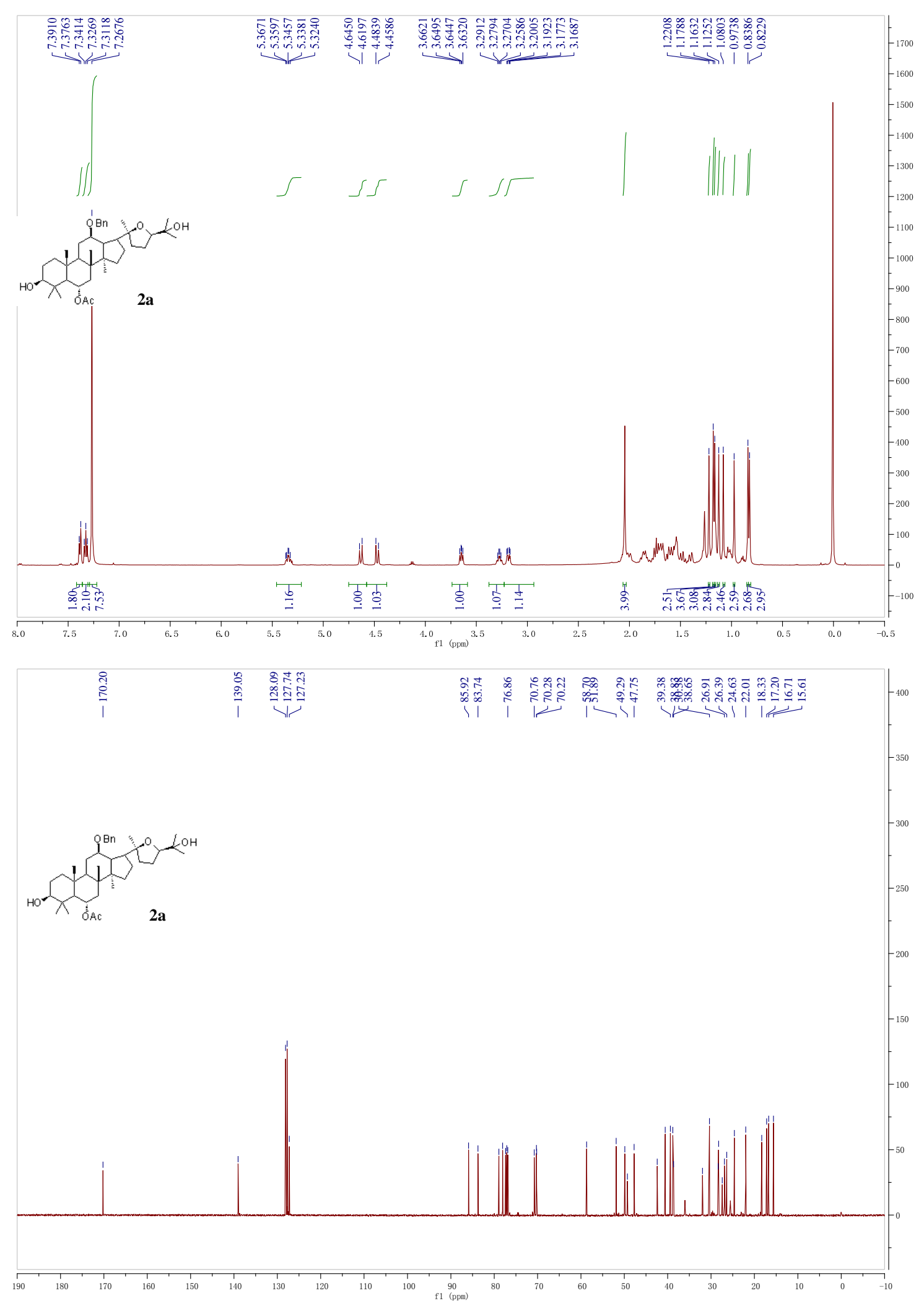

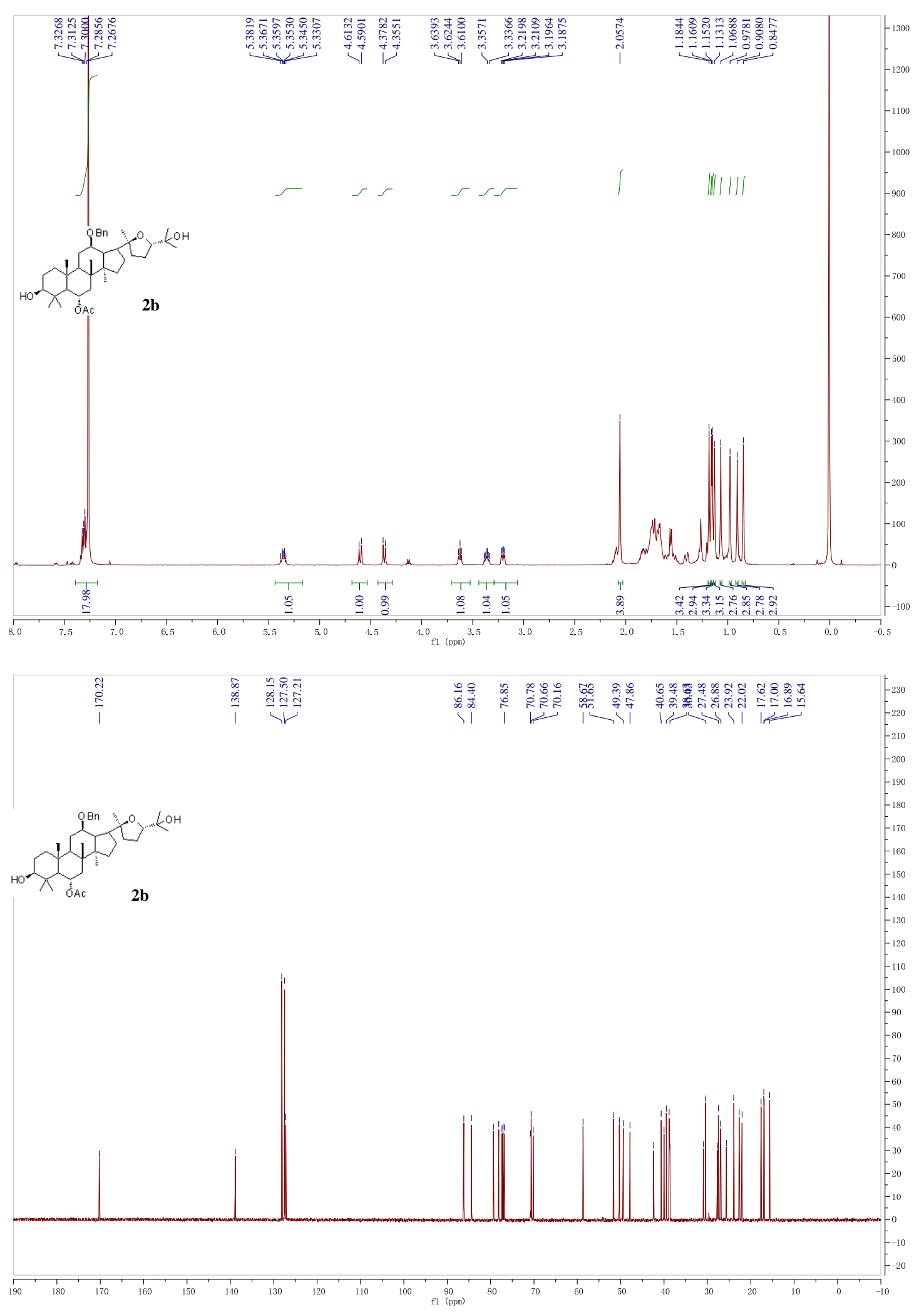

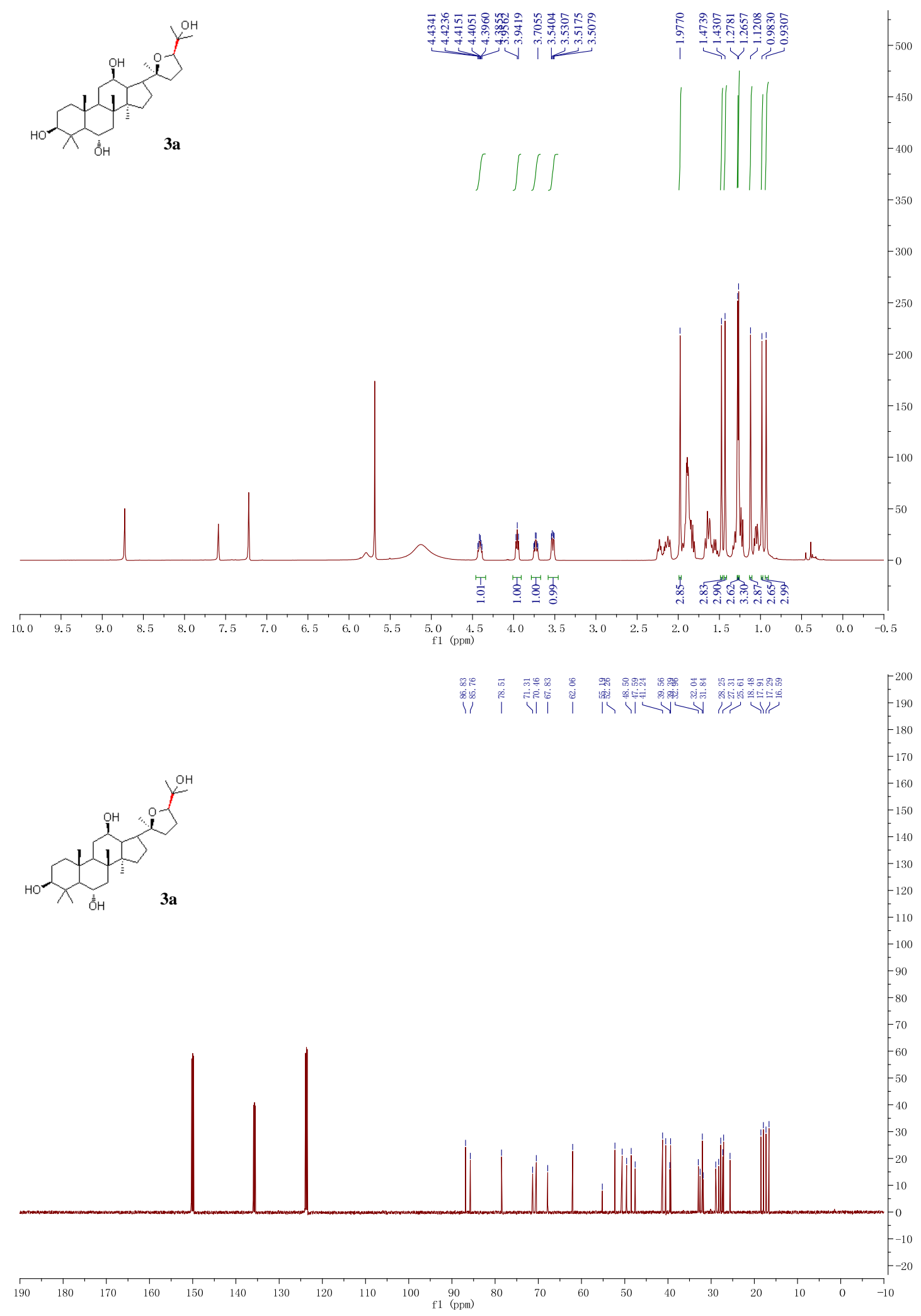

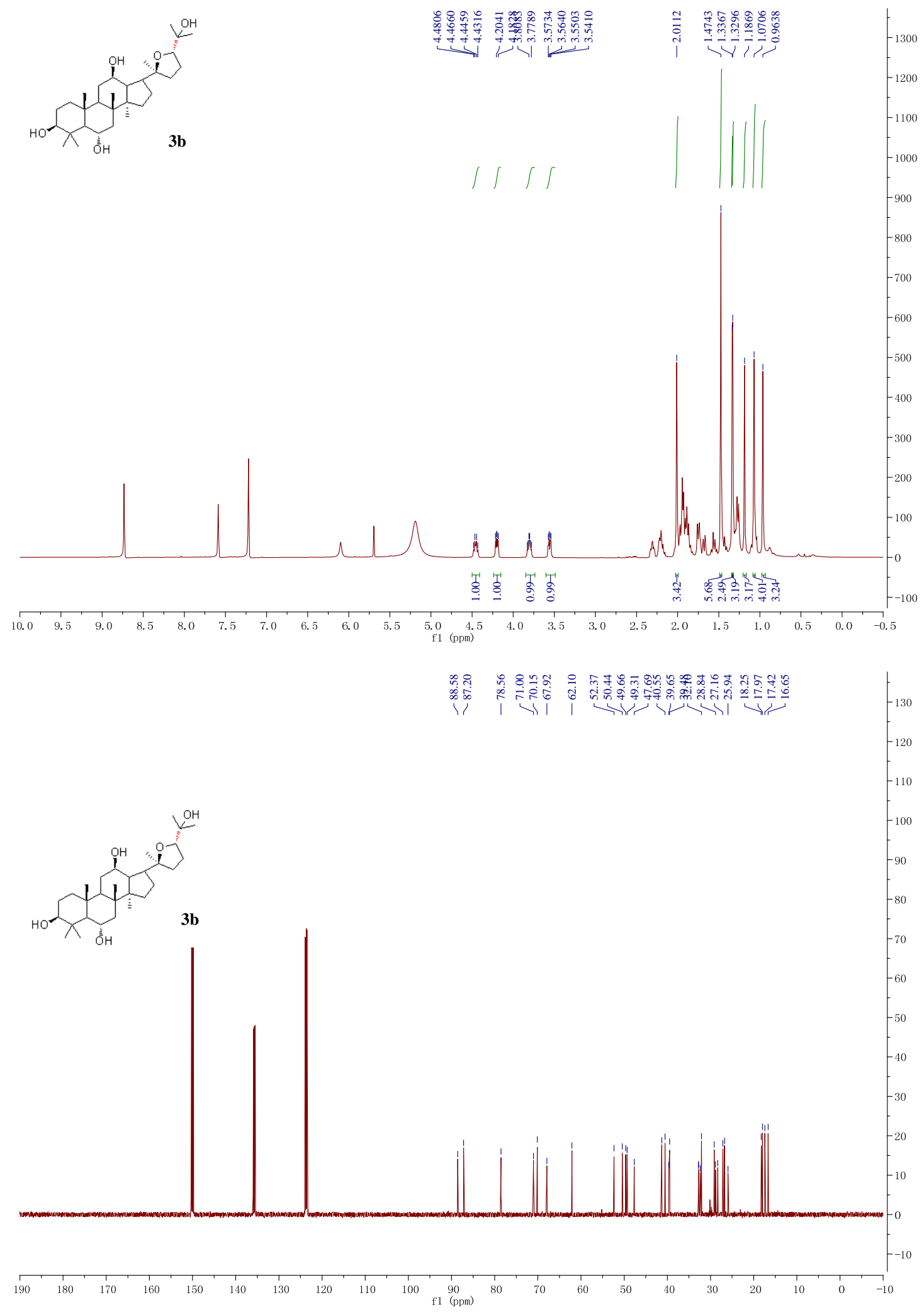


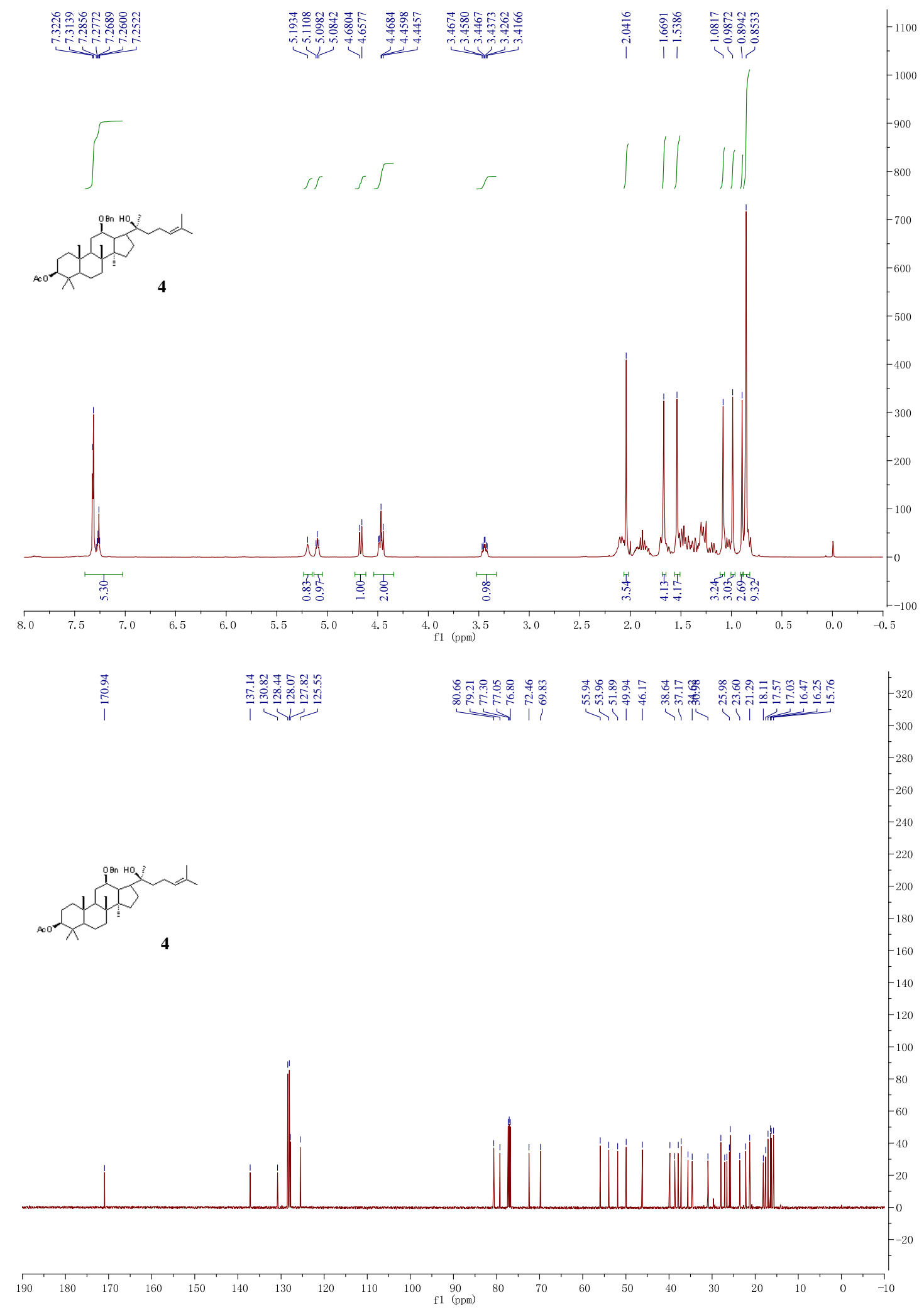



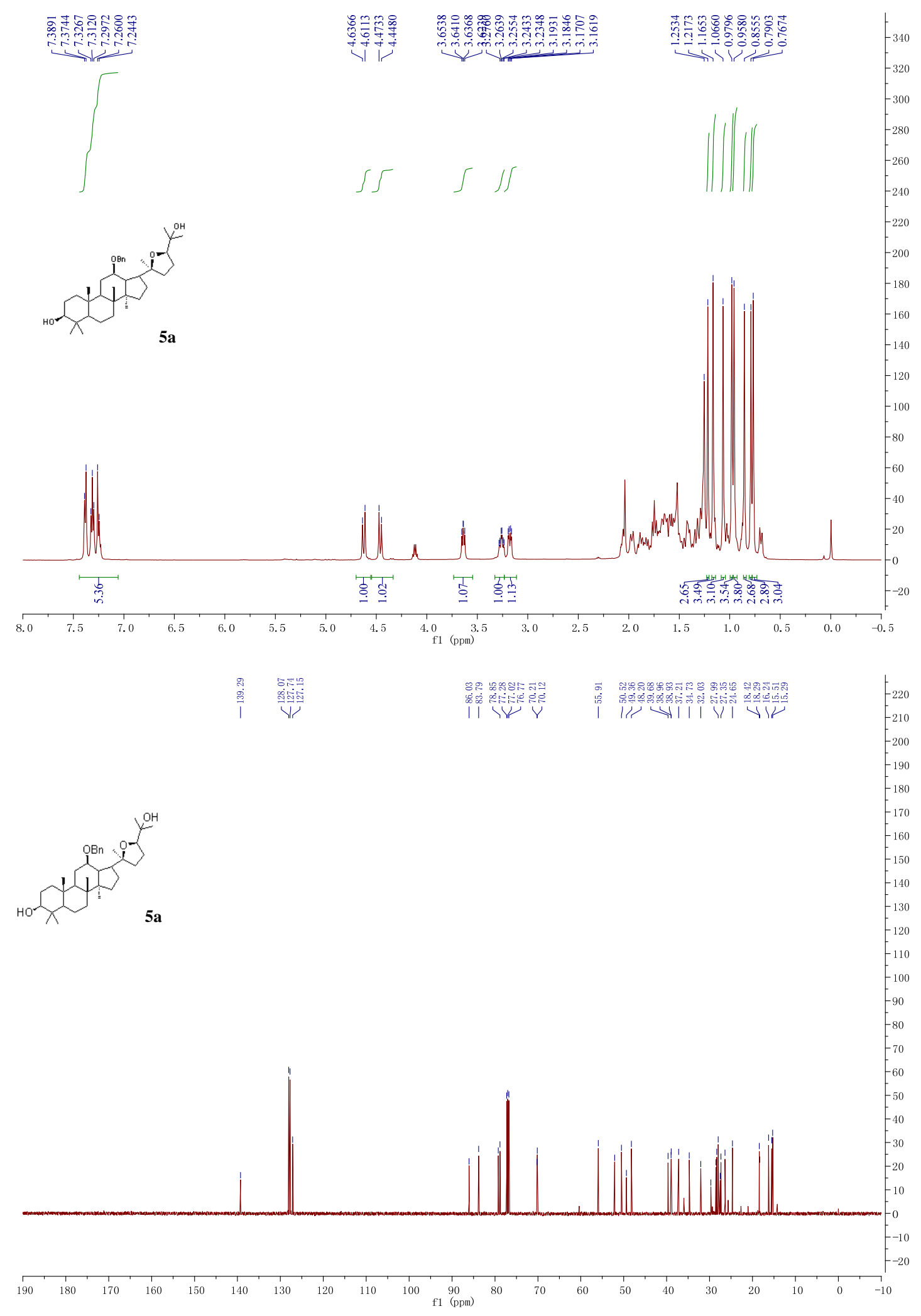


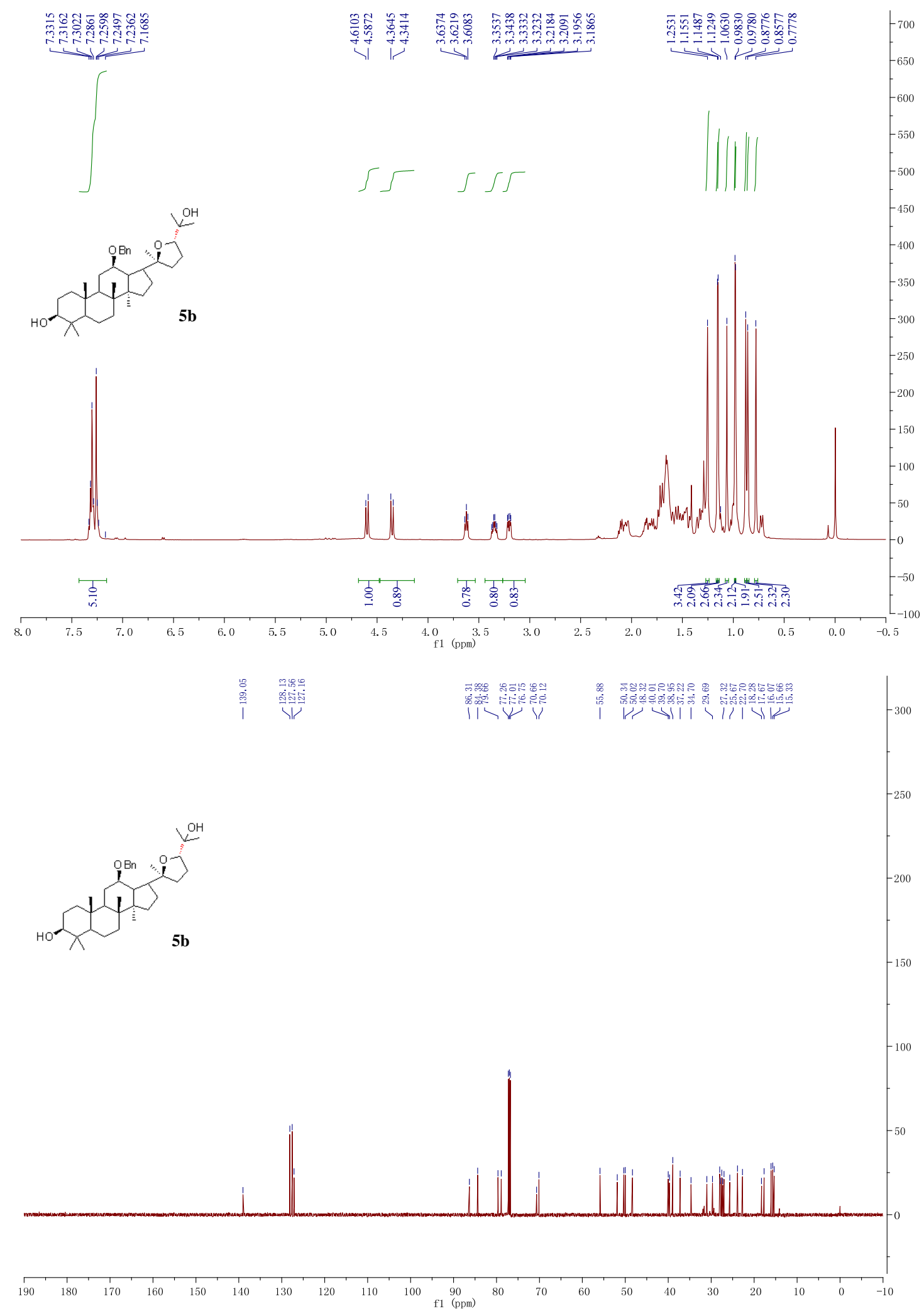



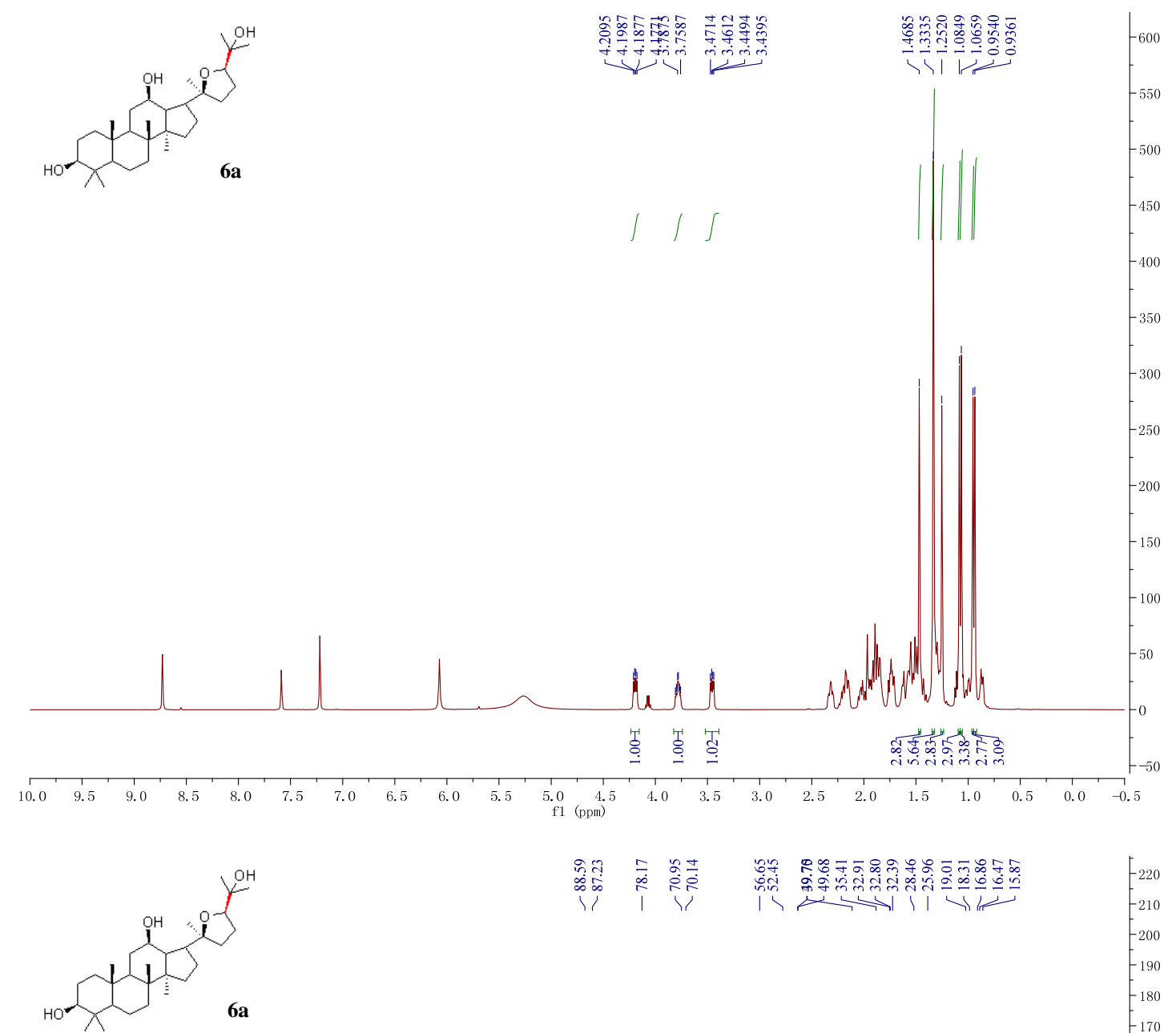

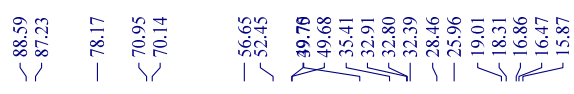

-220
-210
-200
-190
-180
-170
-160
-150
-140
-130
-120
-110
-100
-90
-80
-70
-60
-50
-40
-30
-20
-10
-10

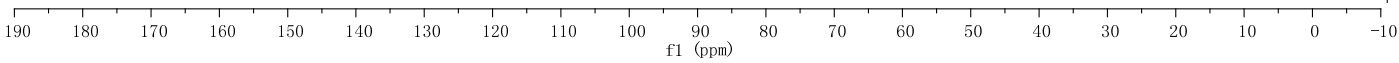



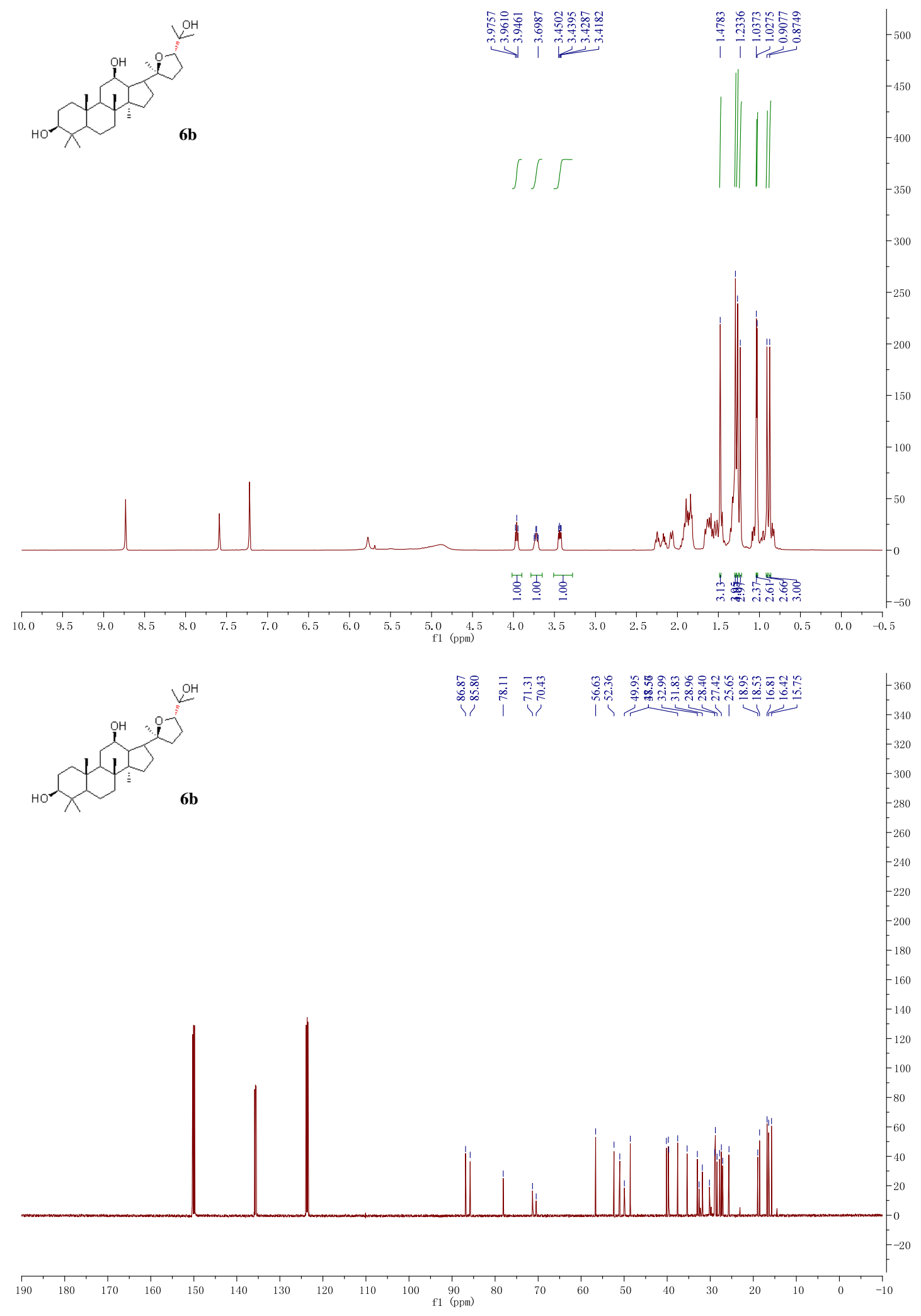

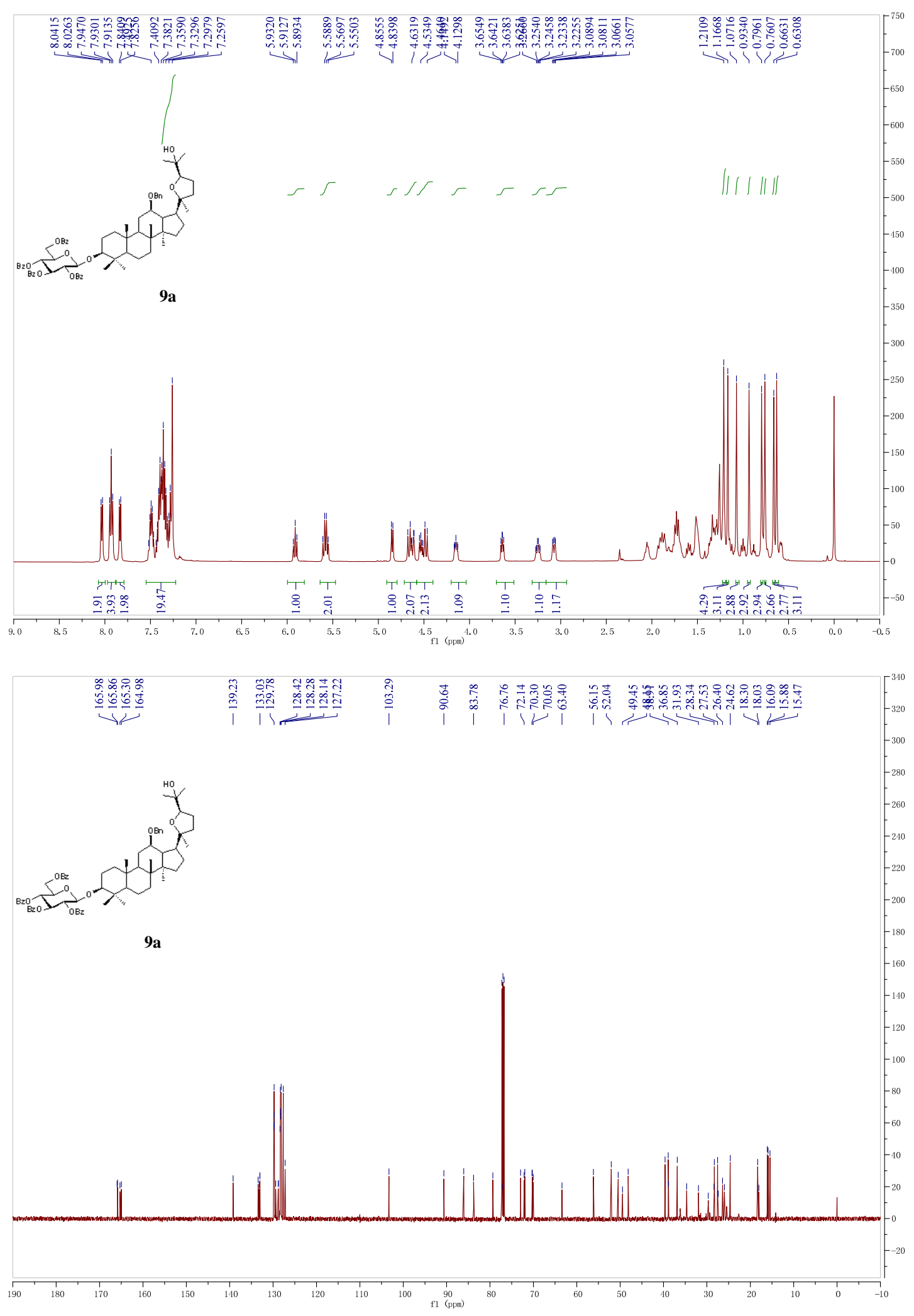


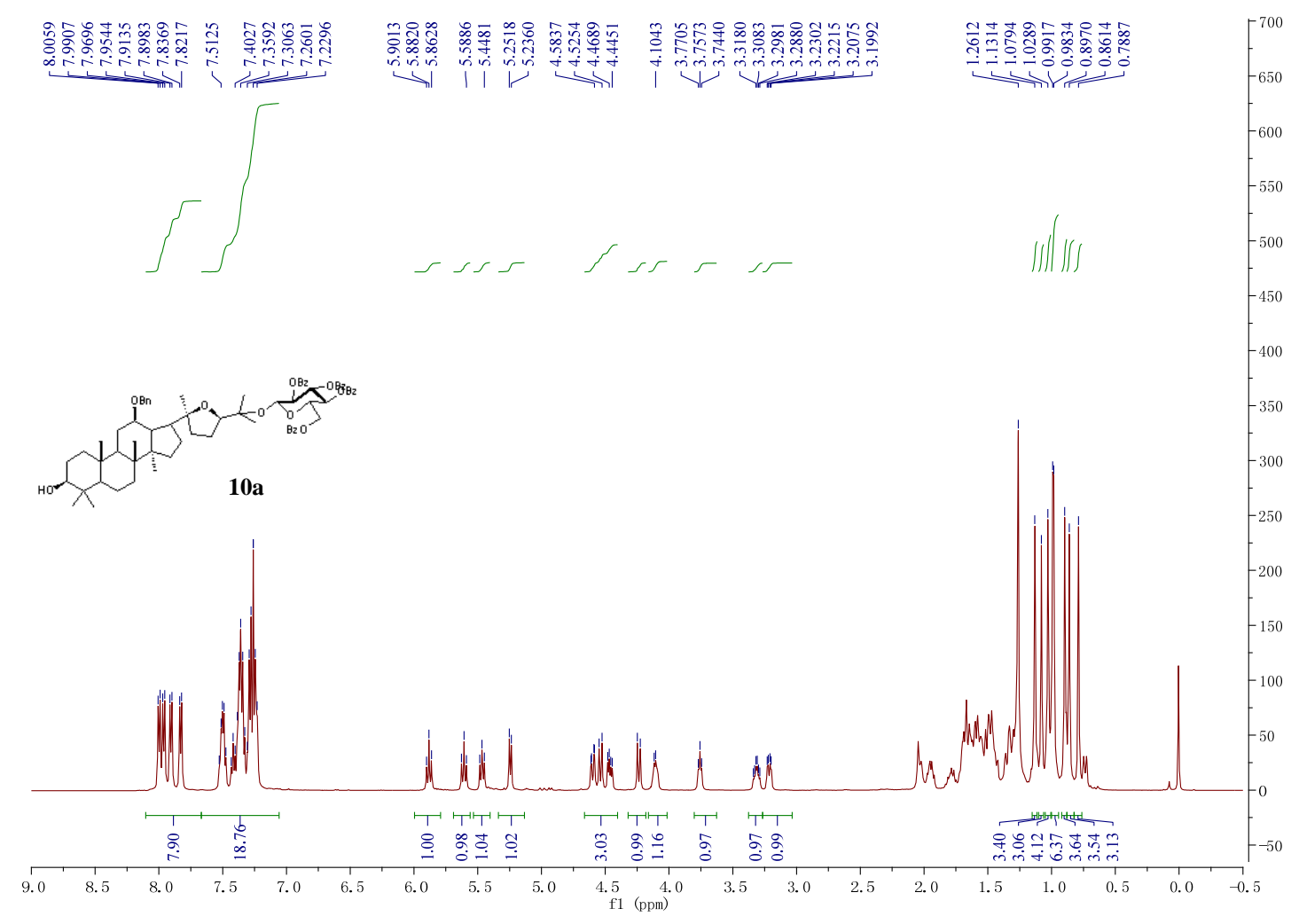

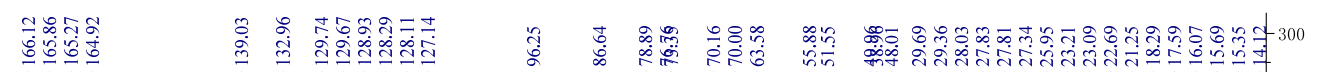

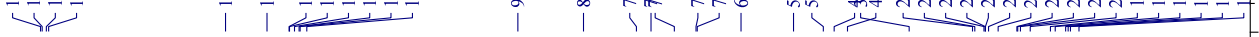

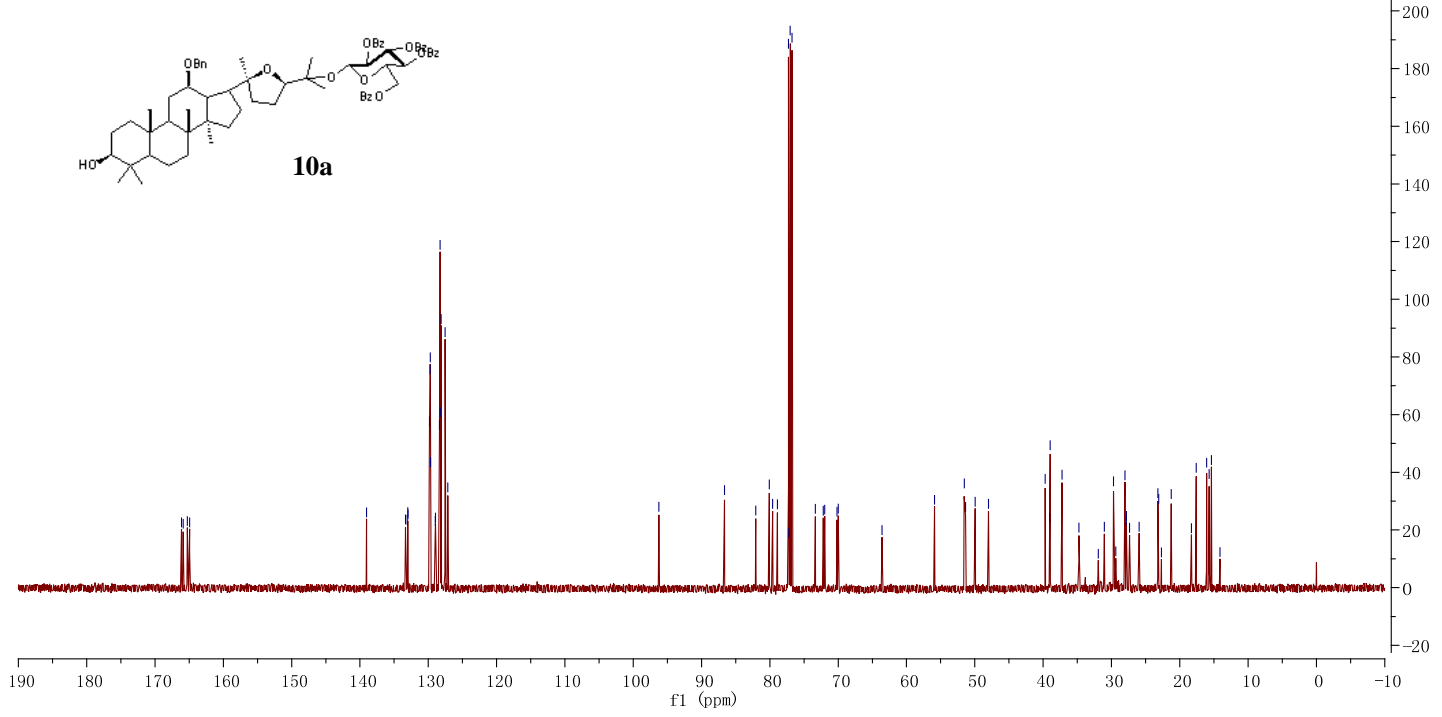



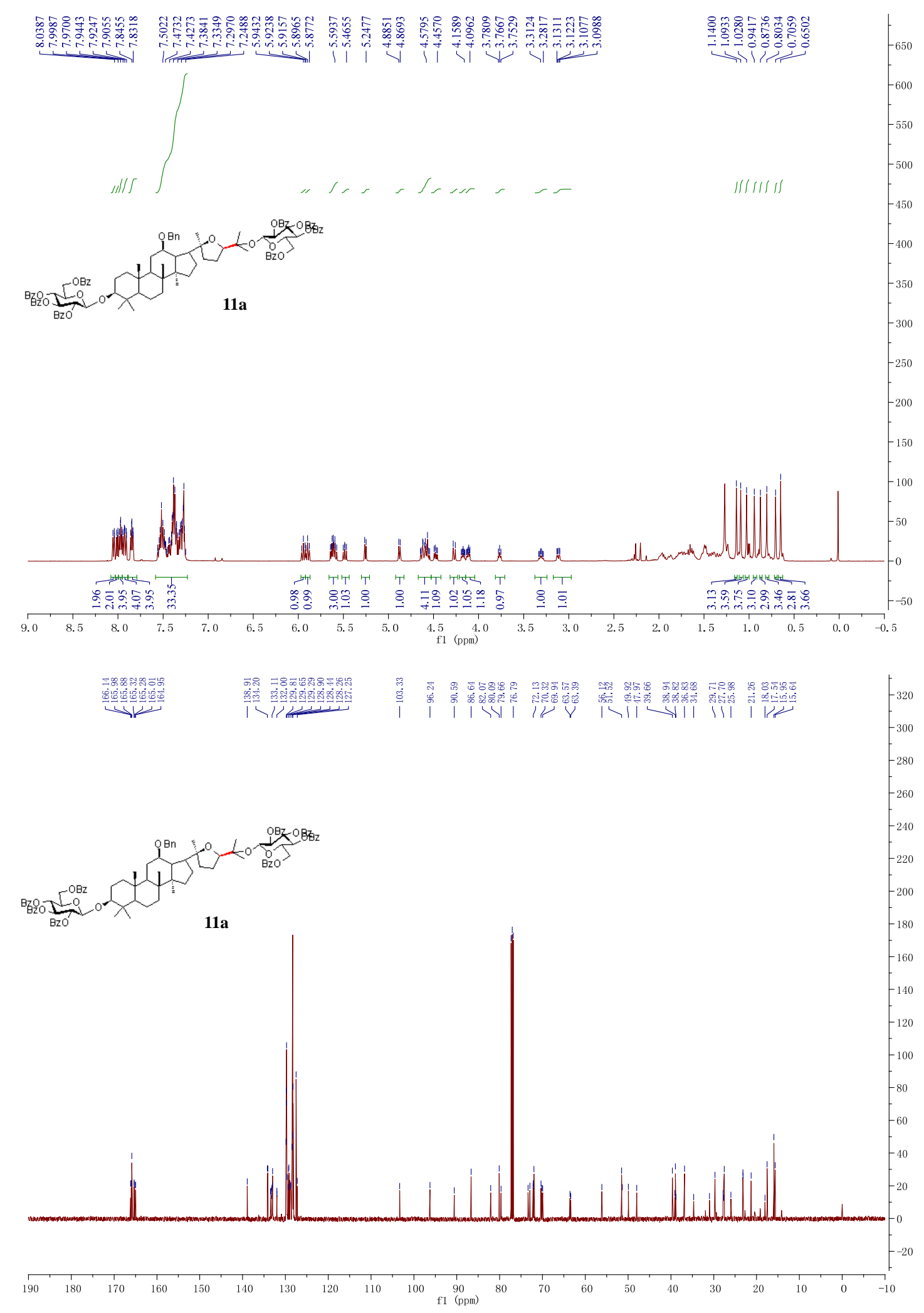


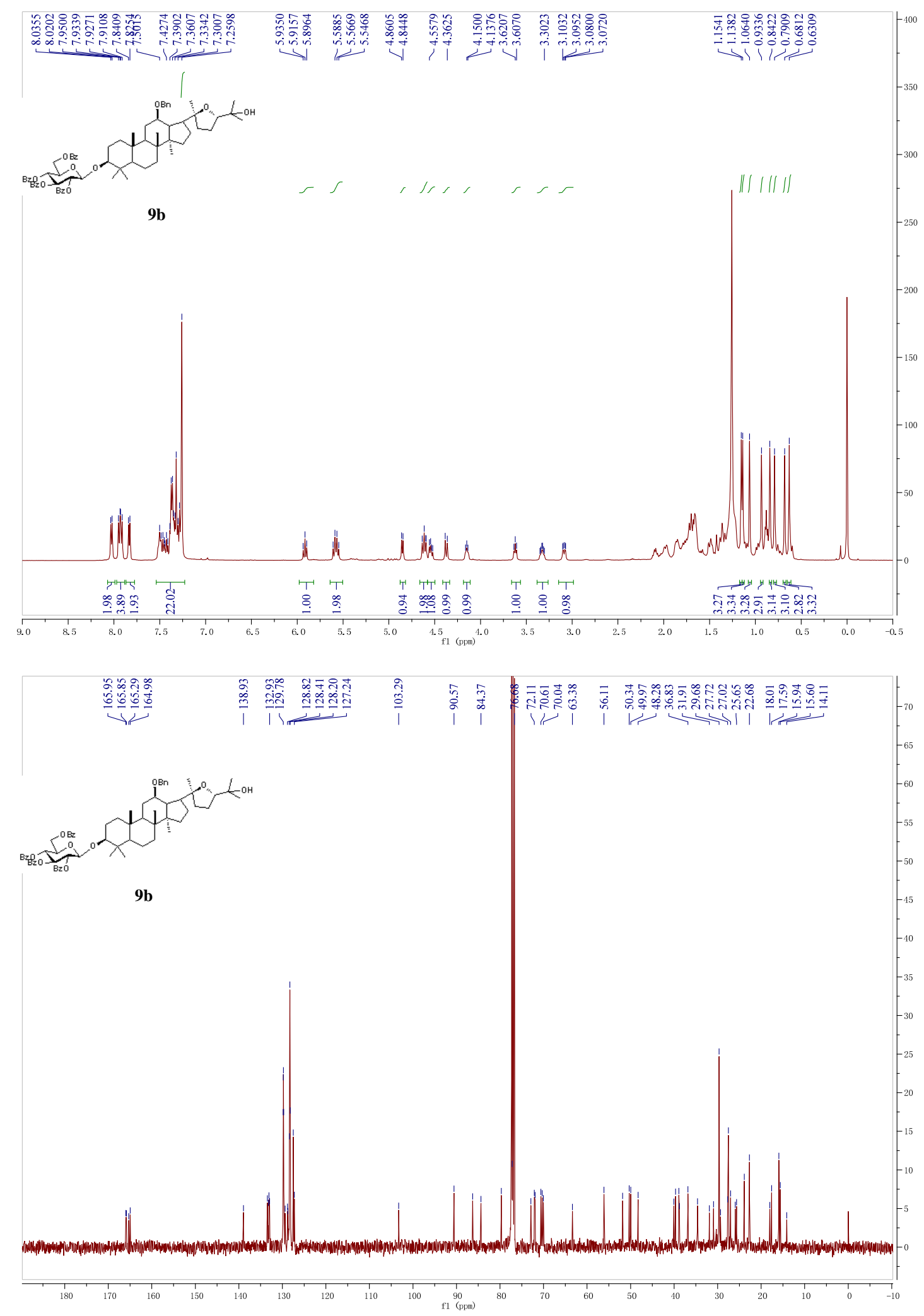




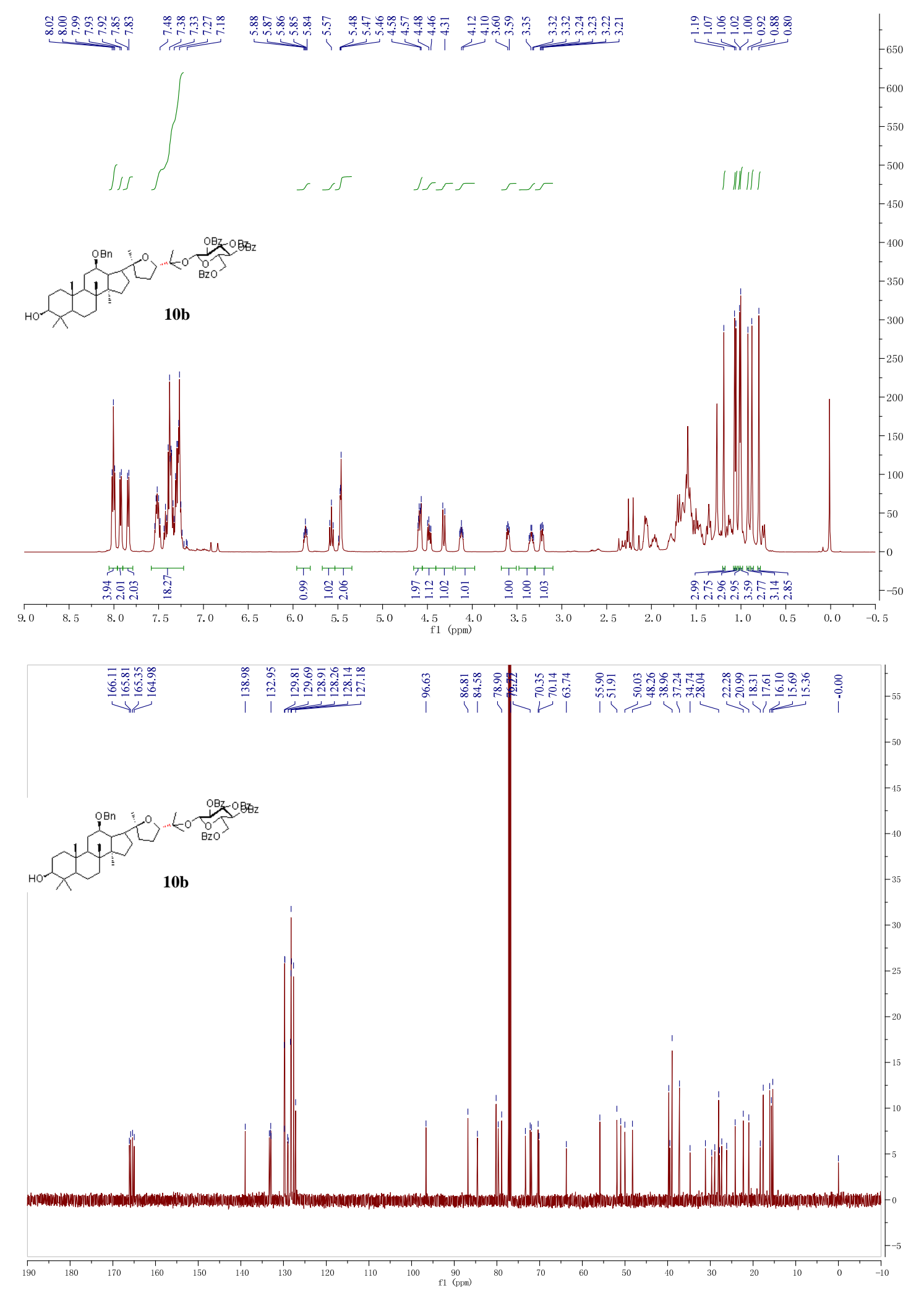




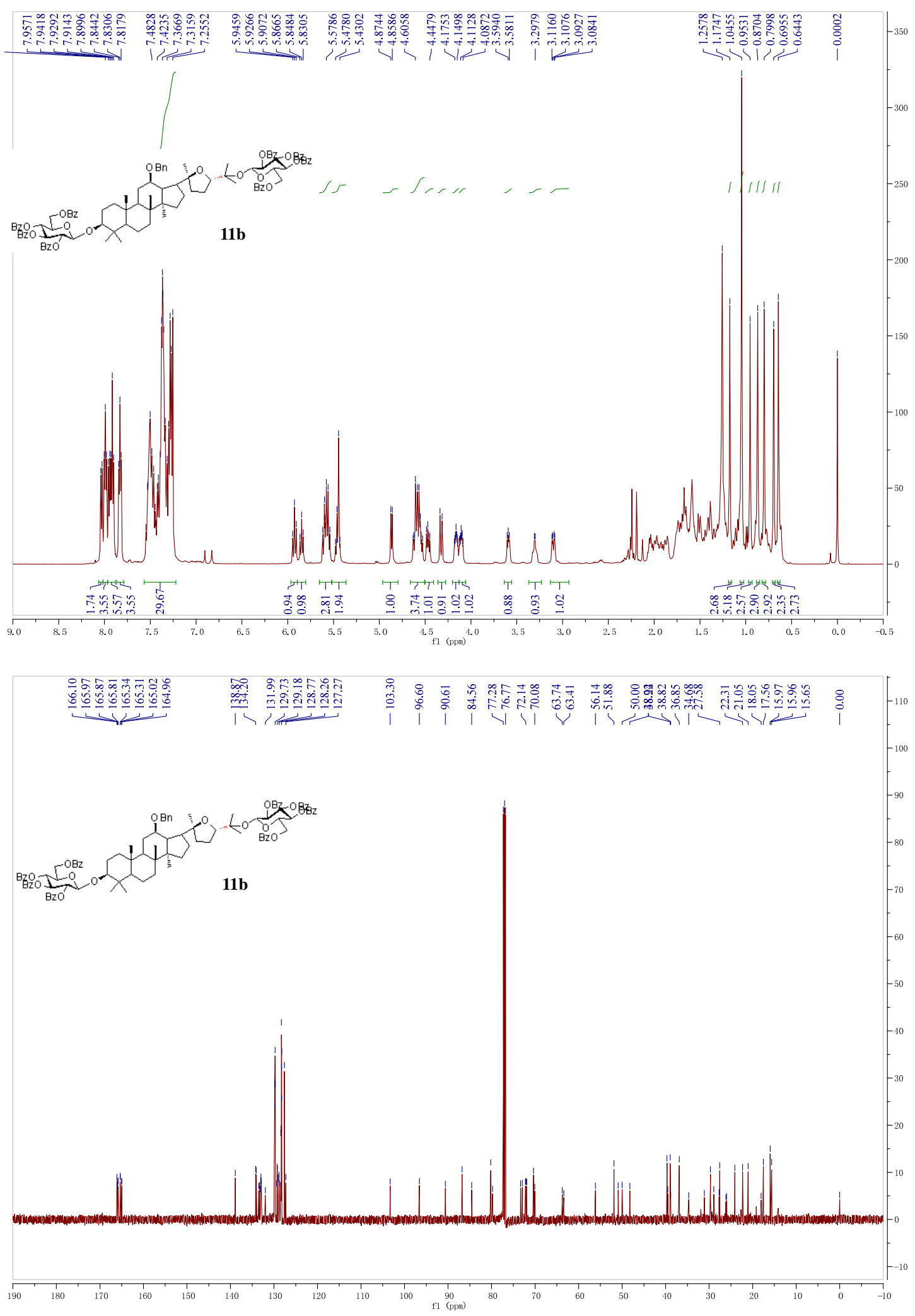



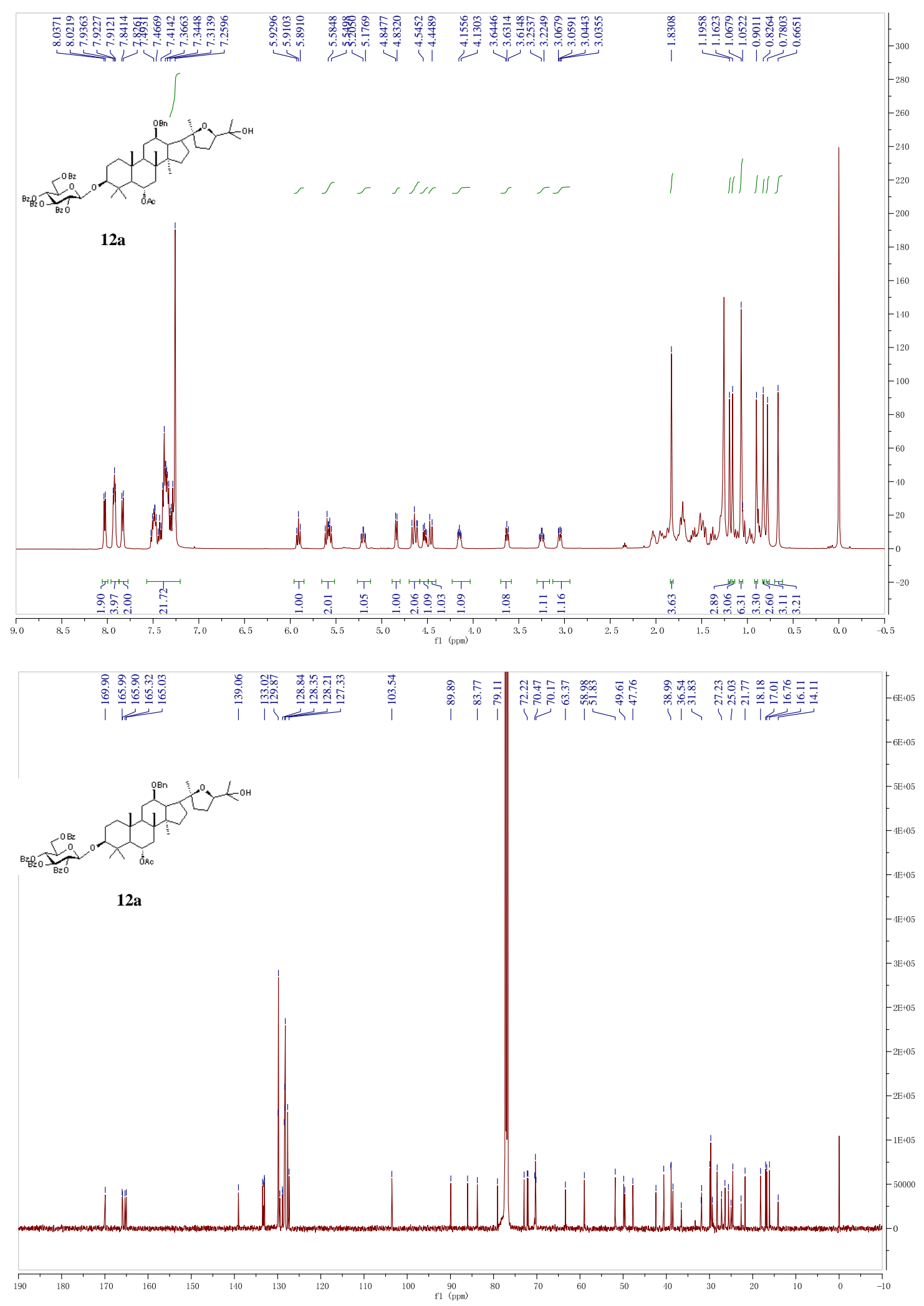

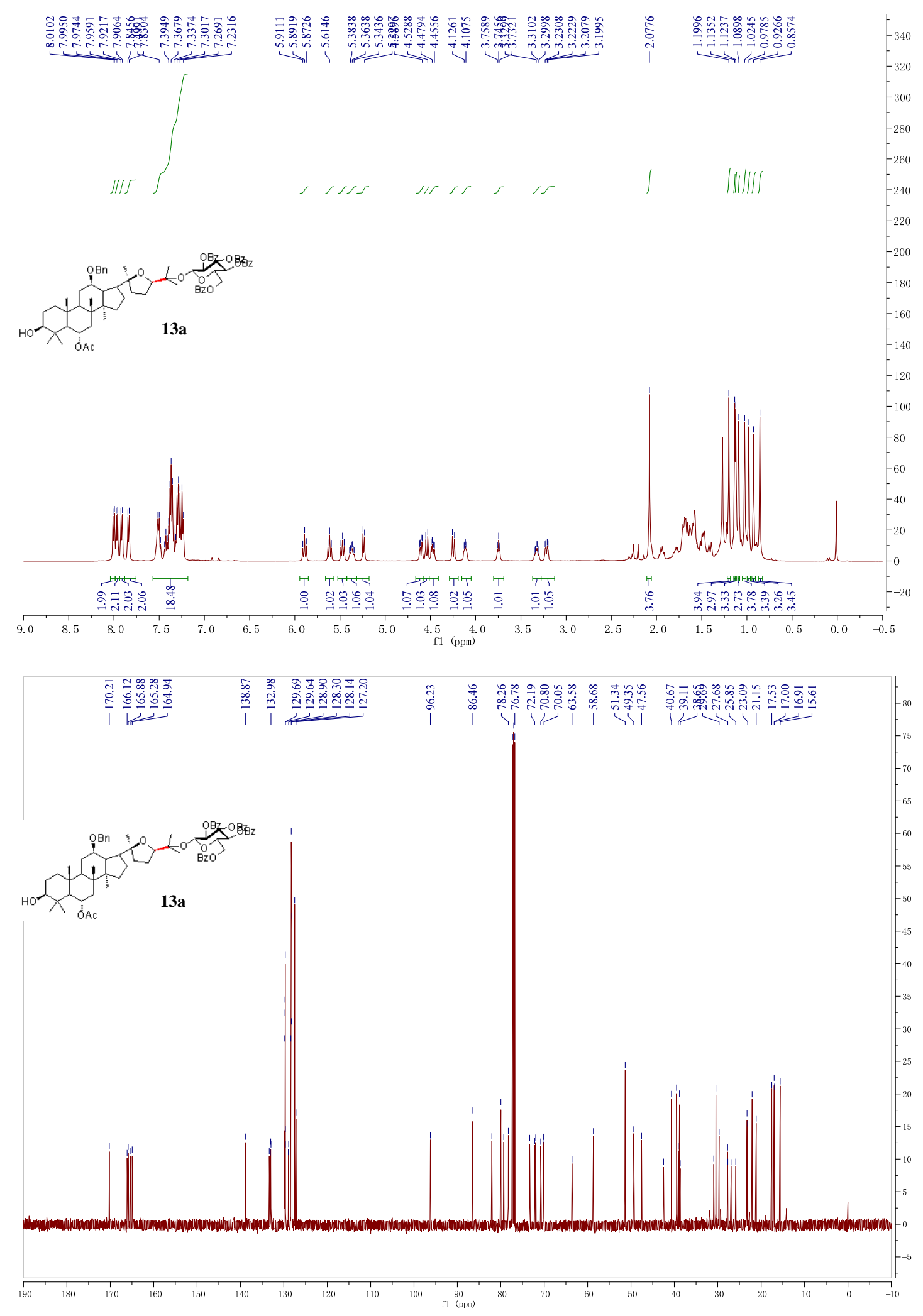


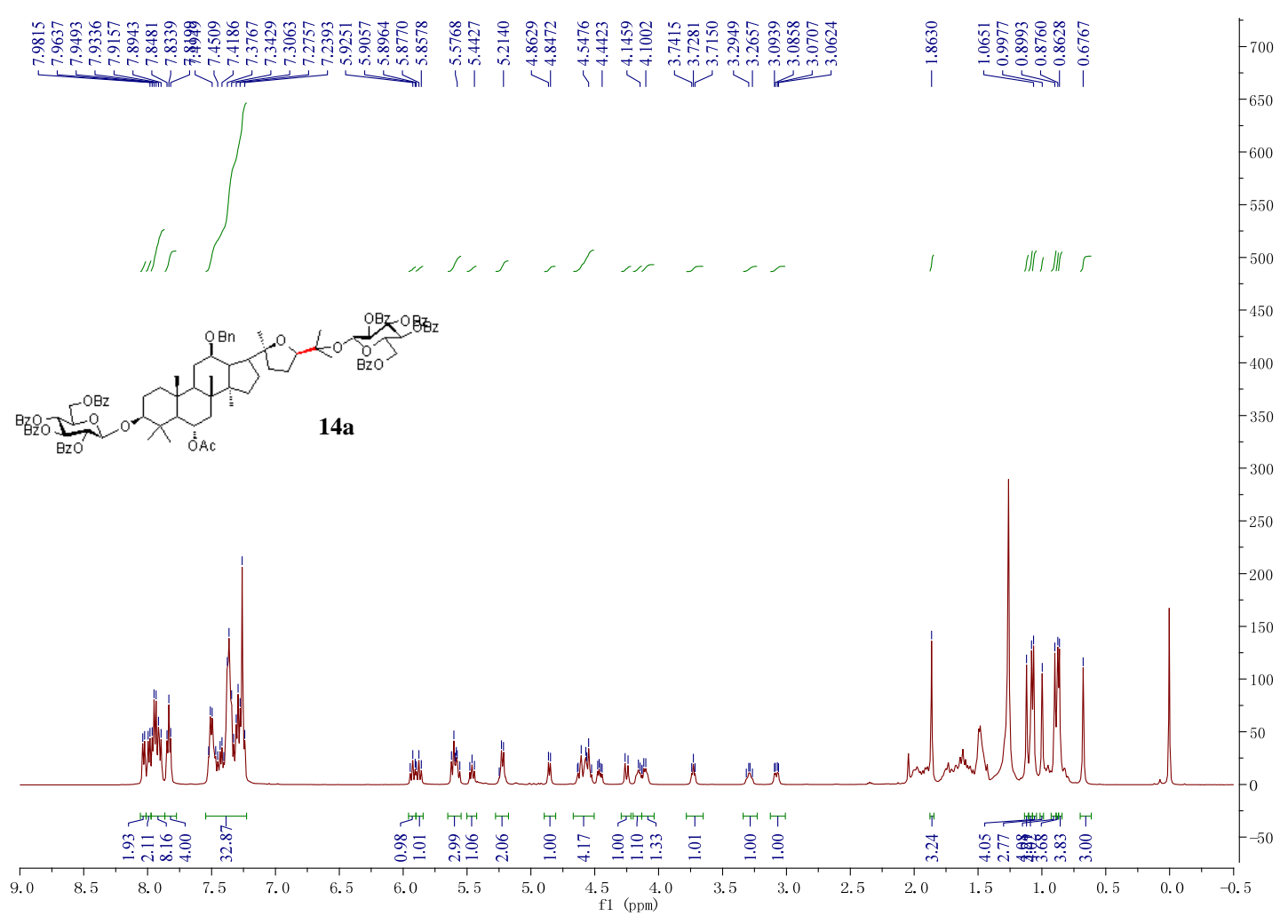

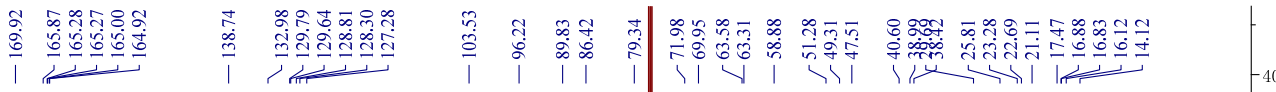
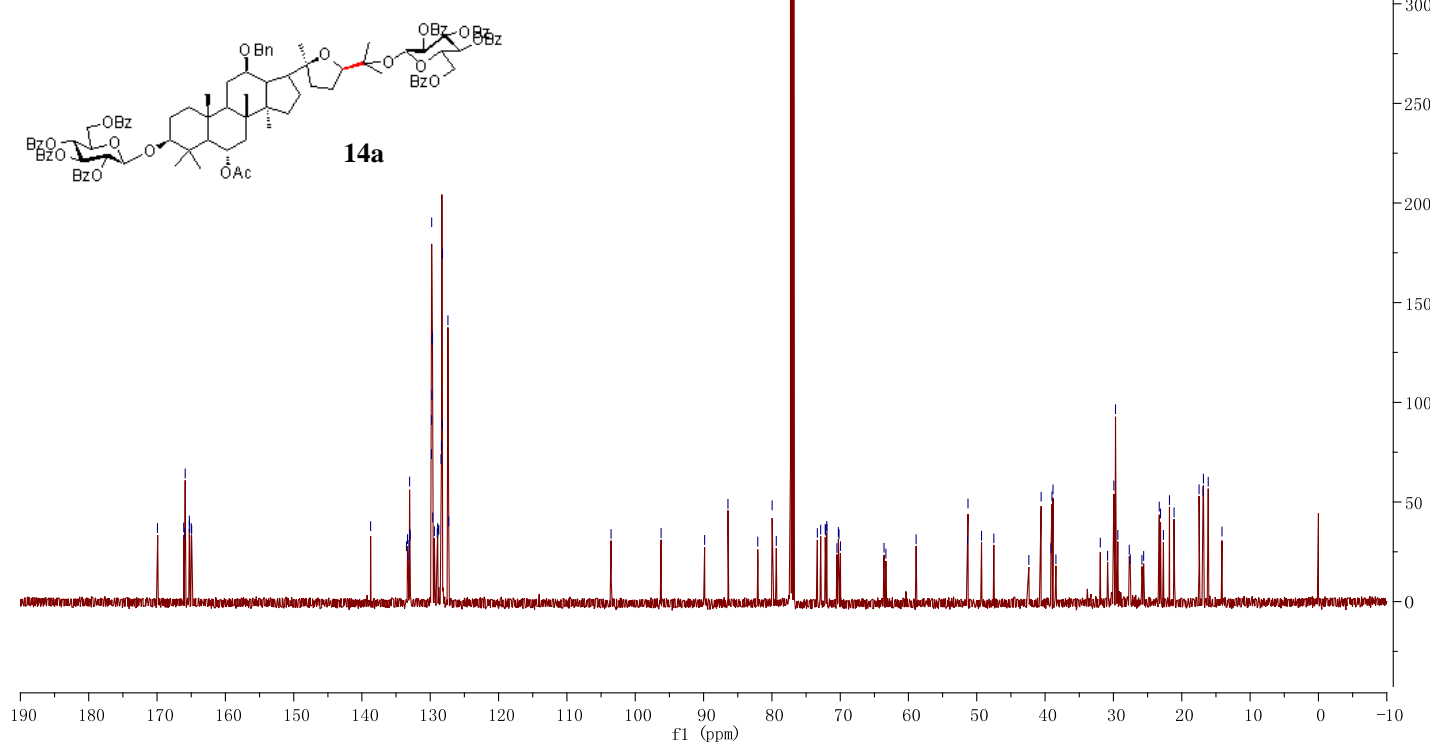


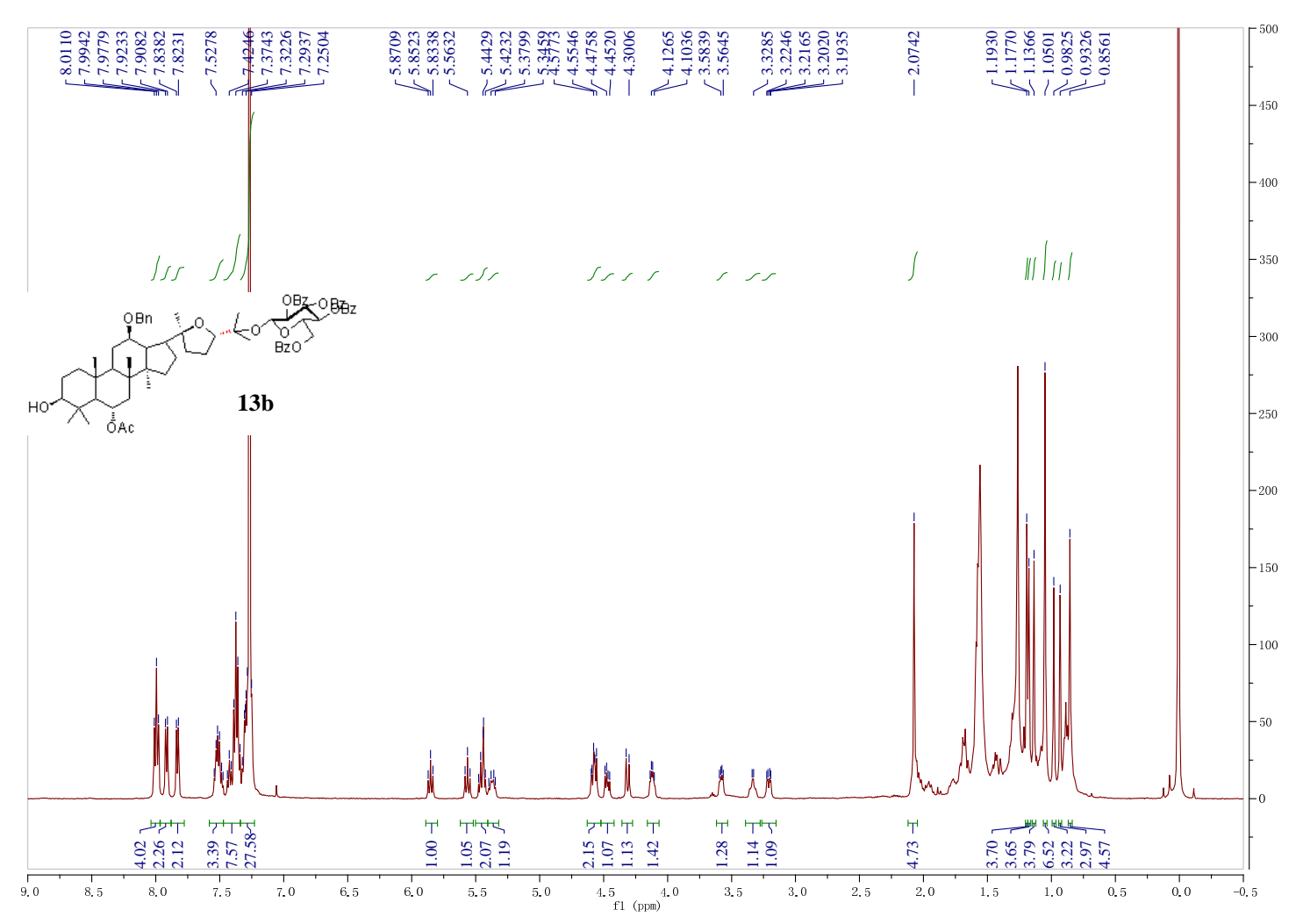

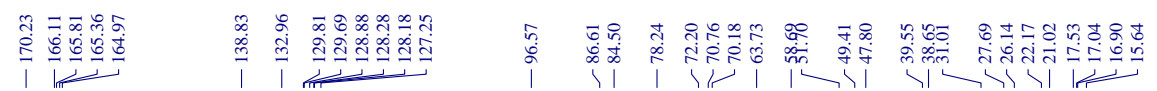
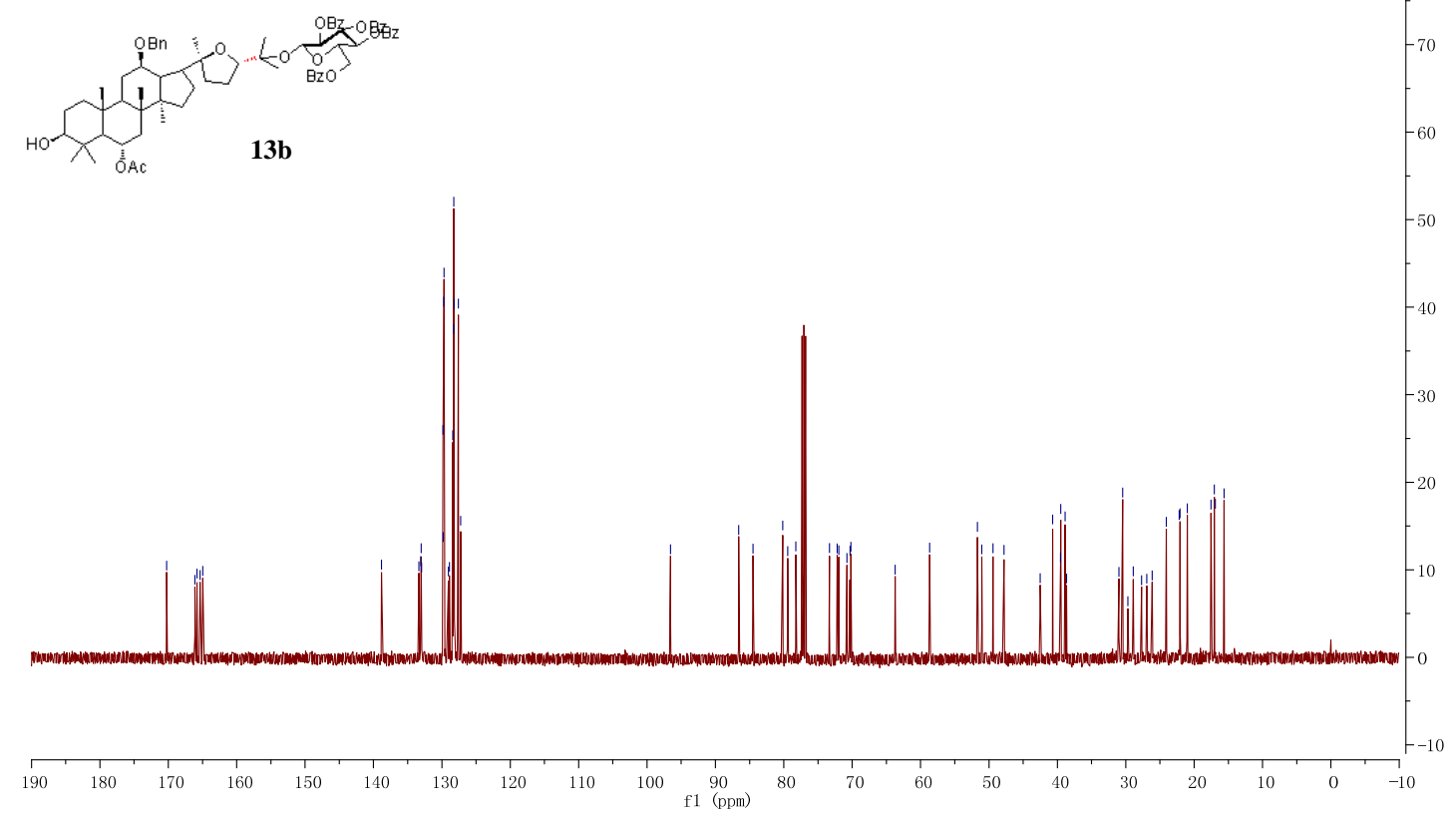

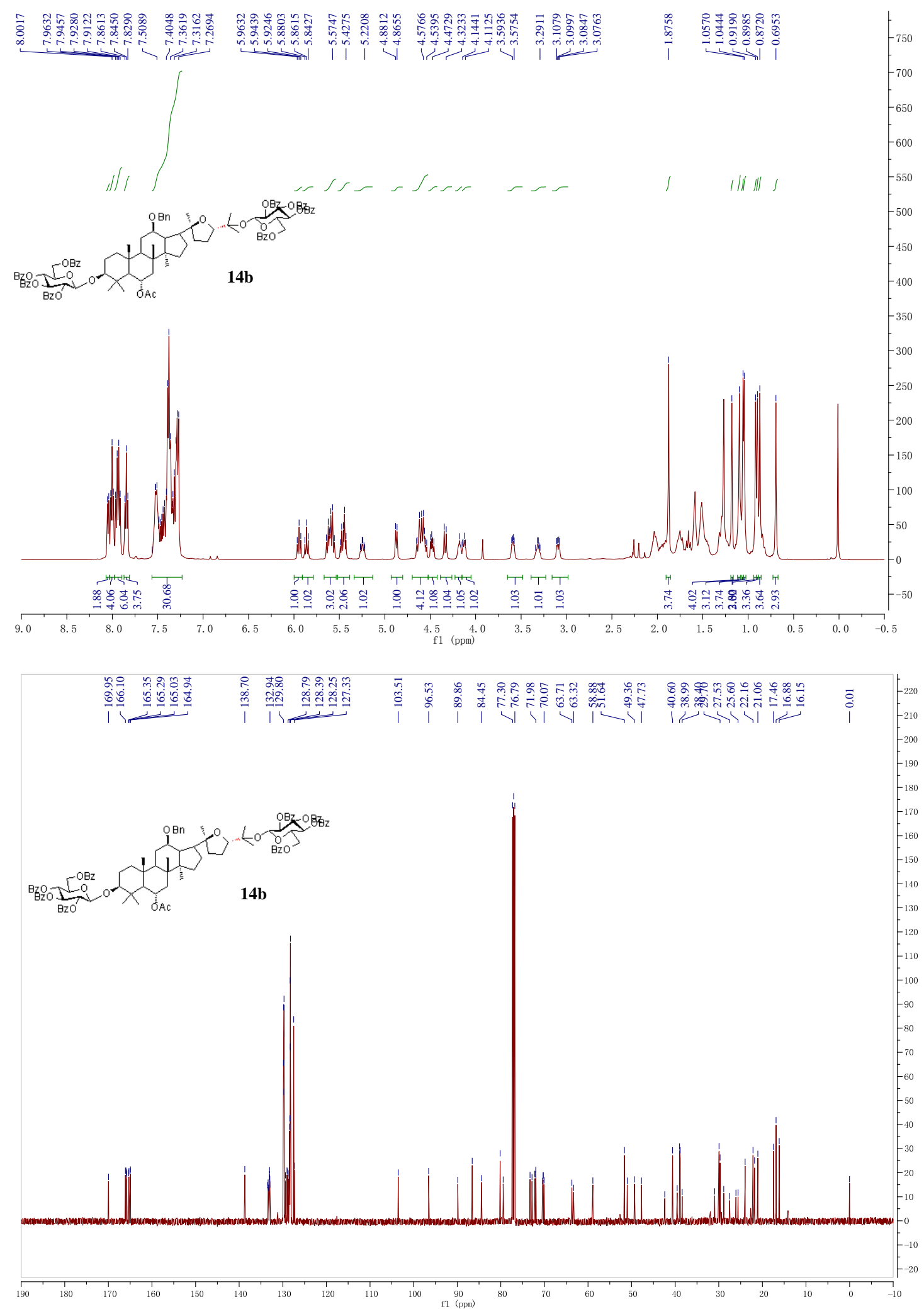


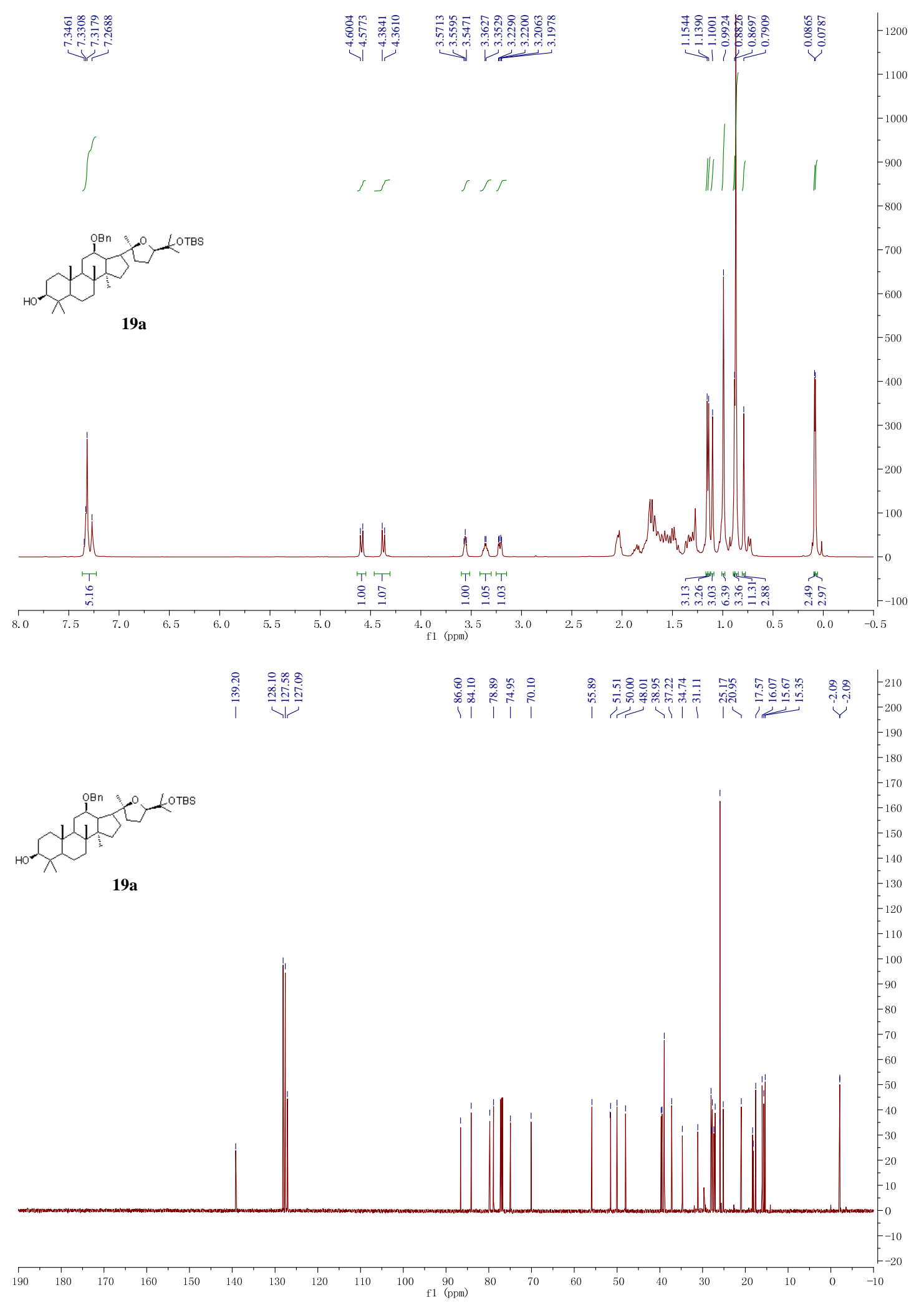




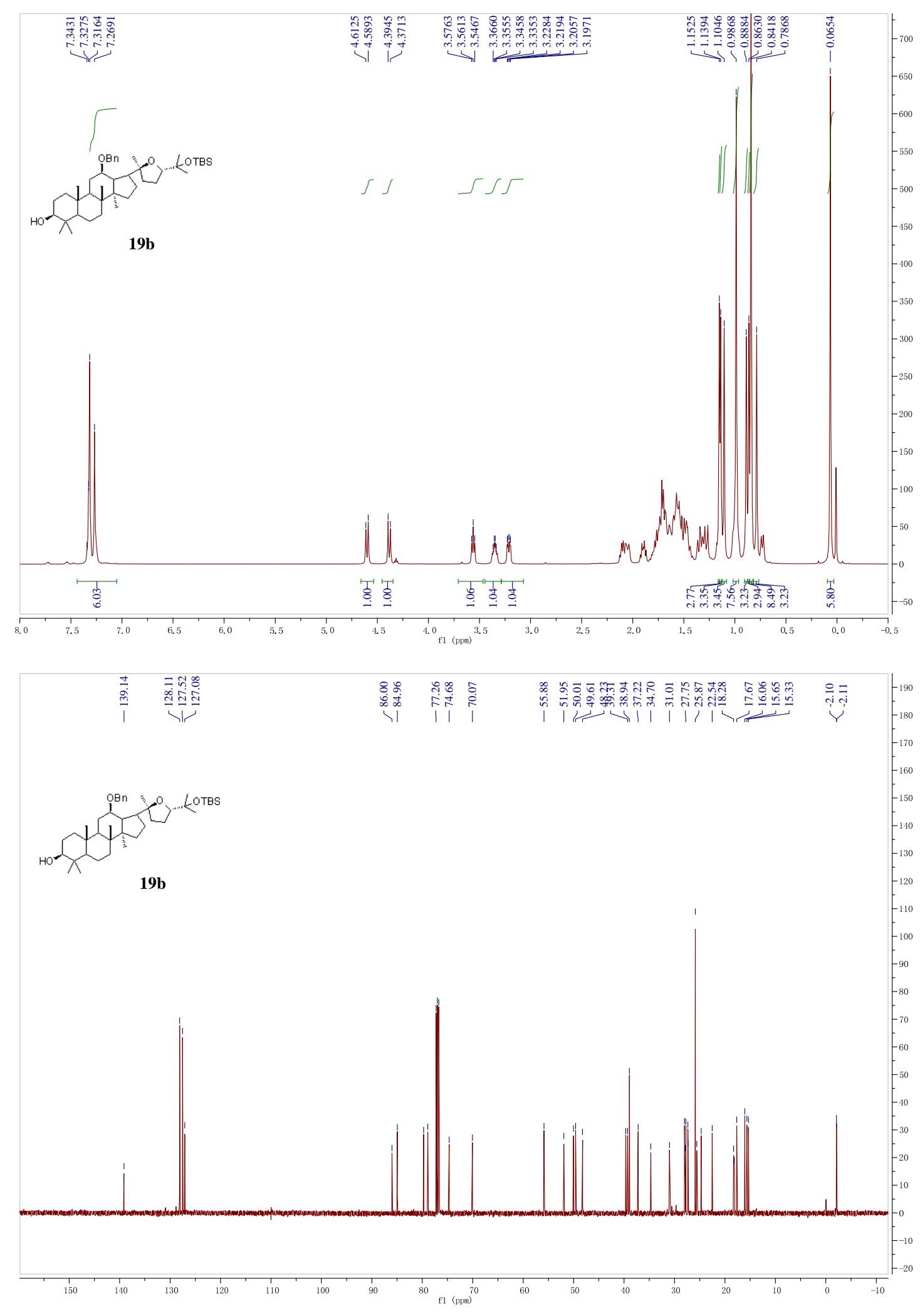



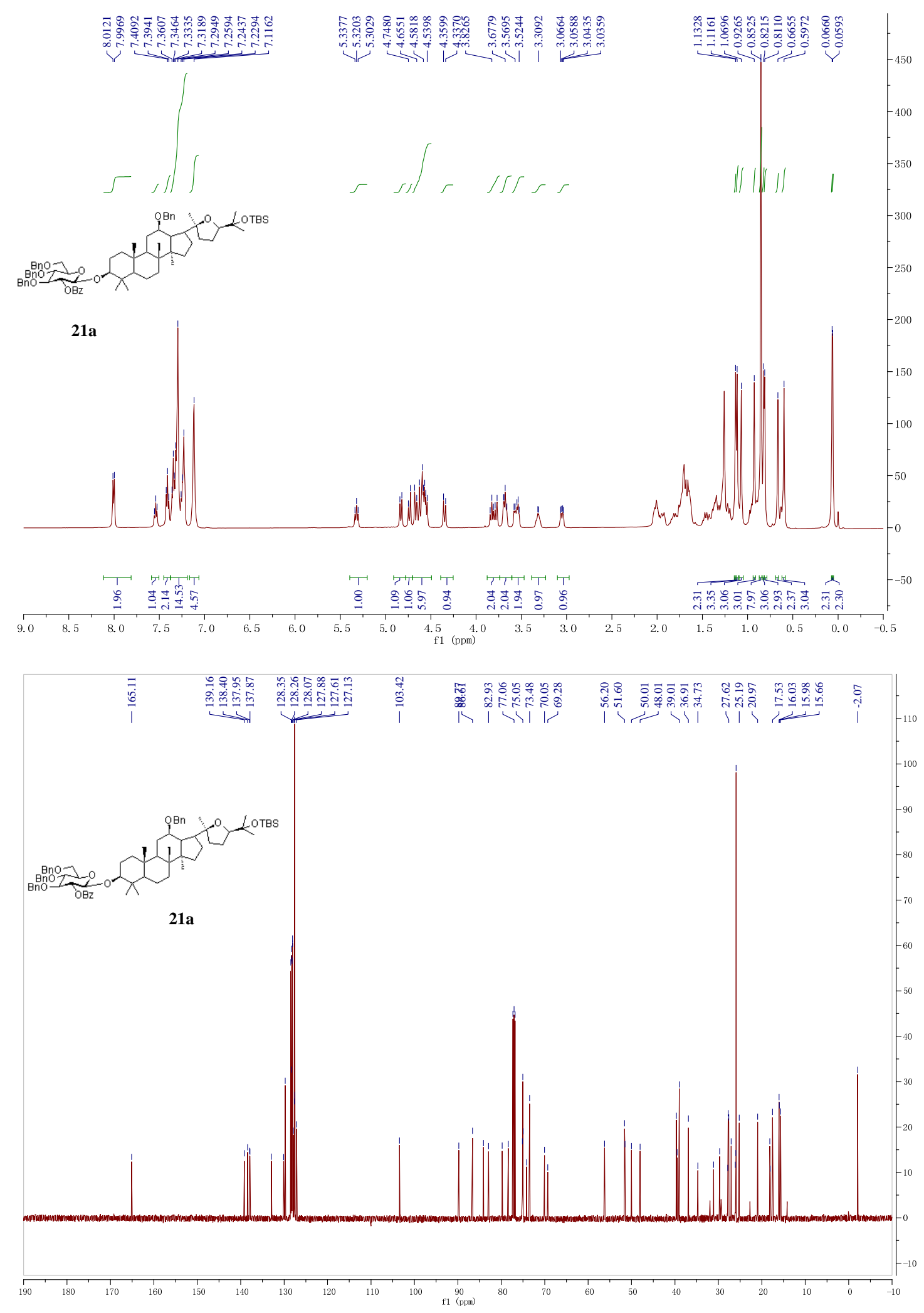

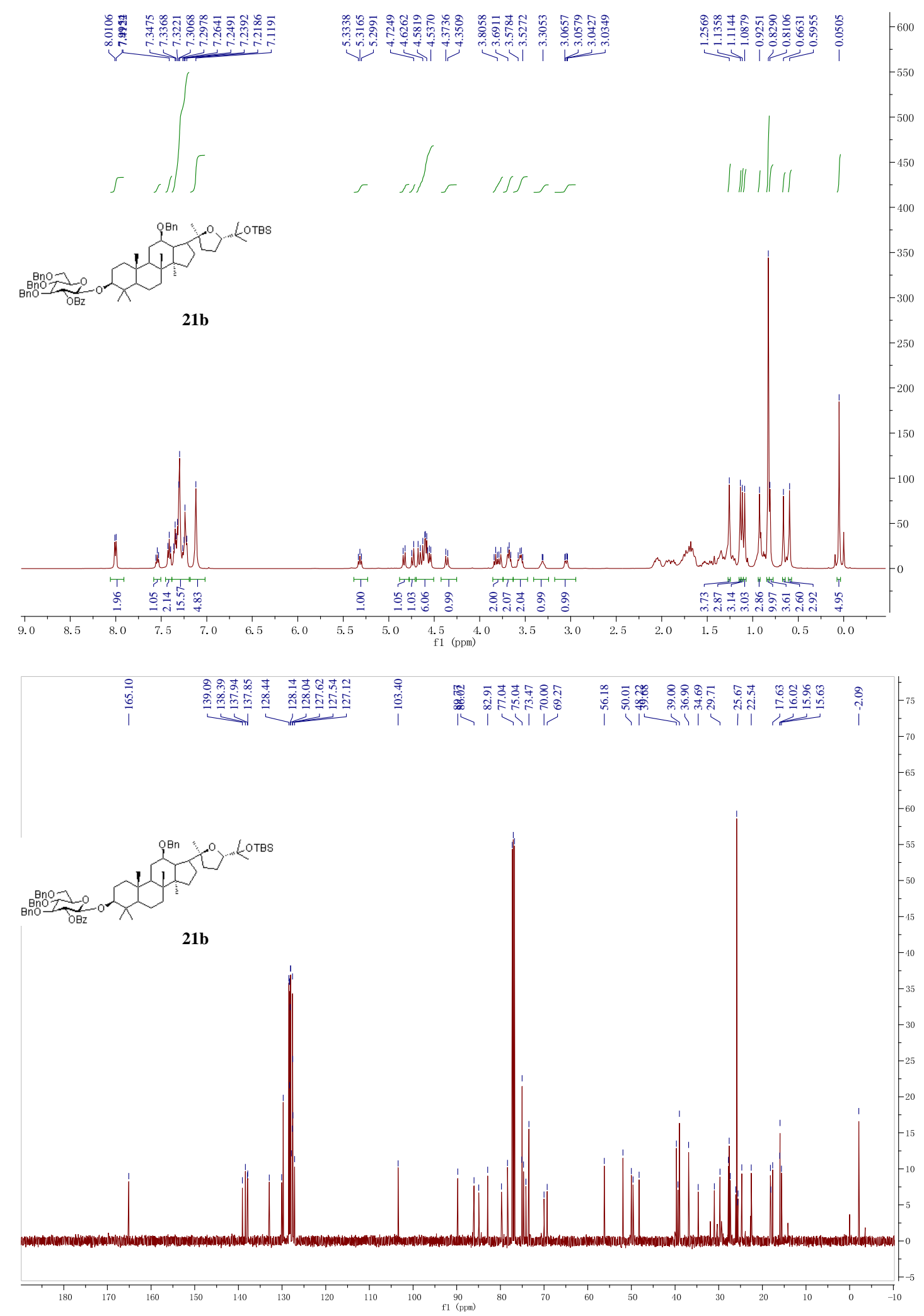

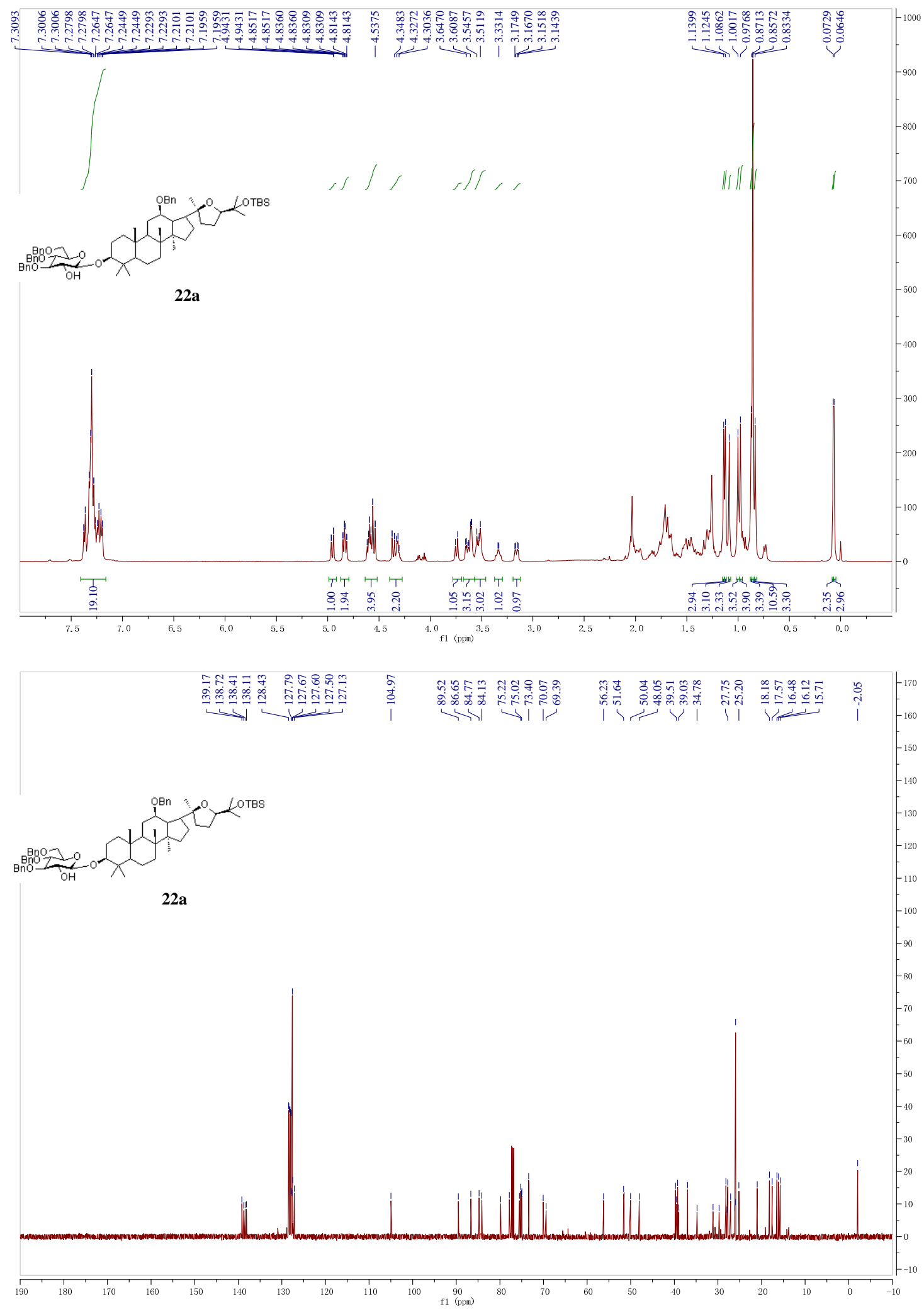


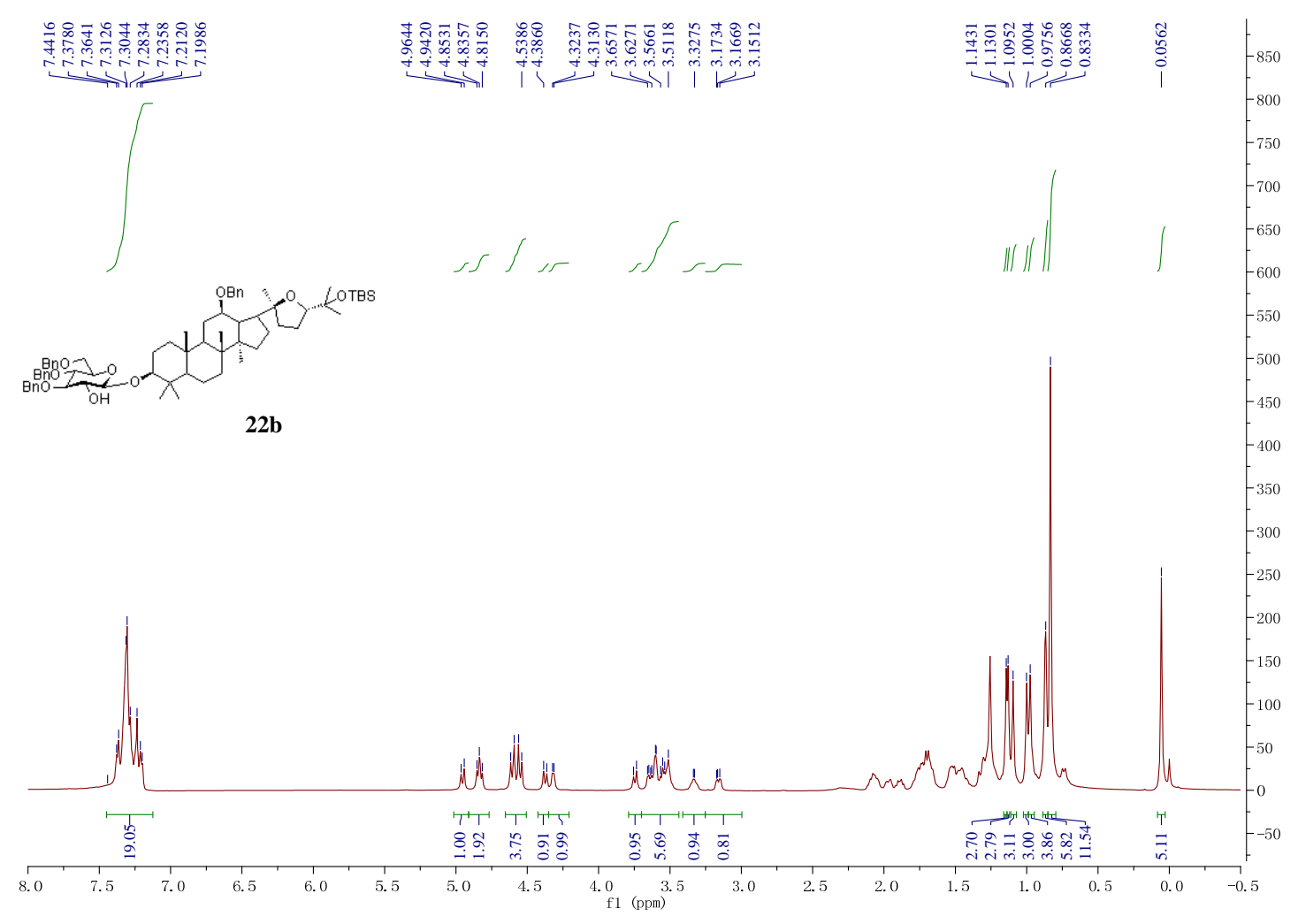

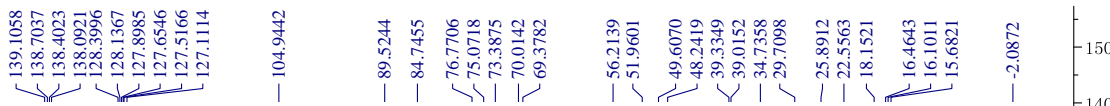

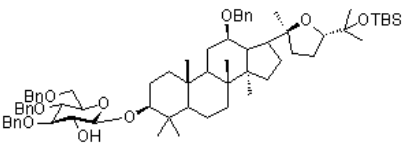

22b

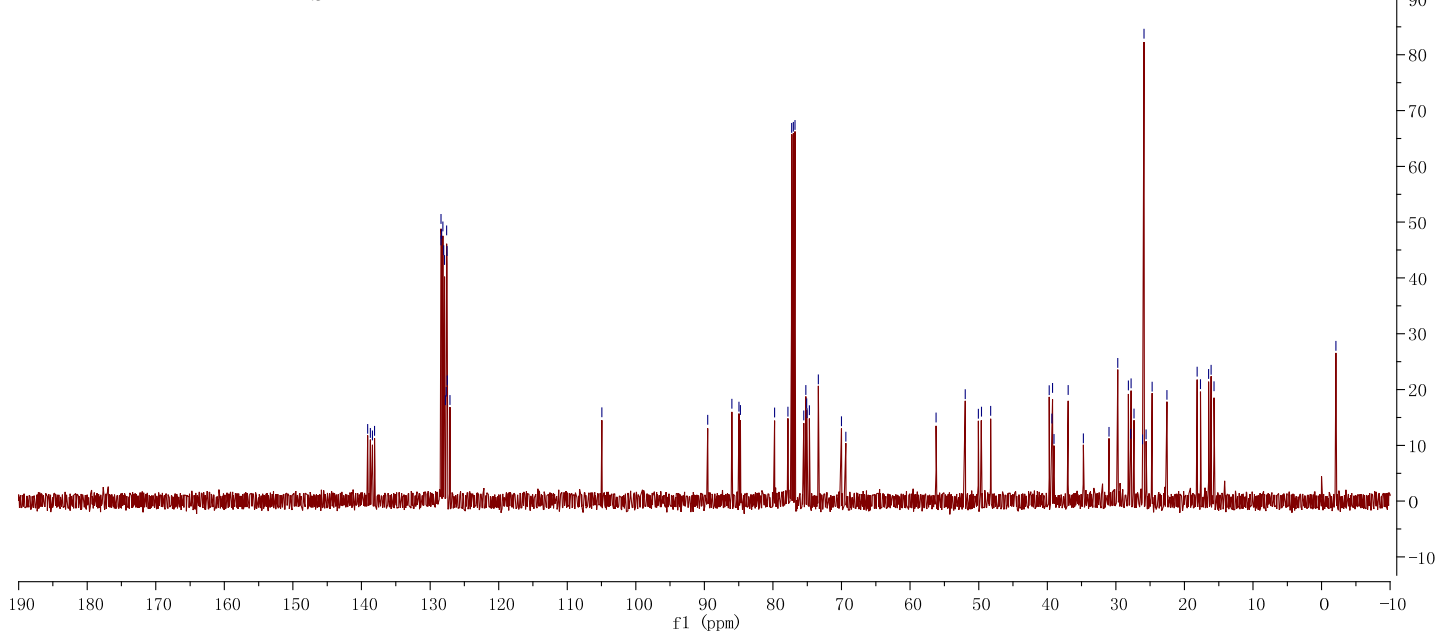




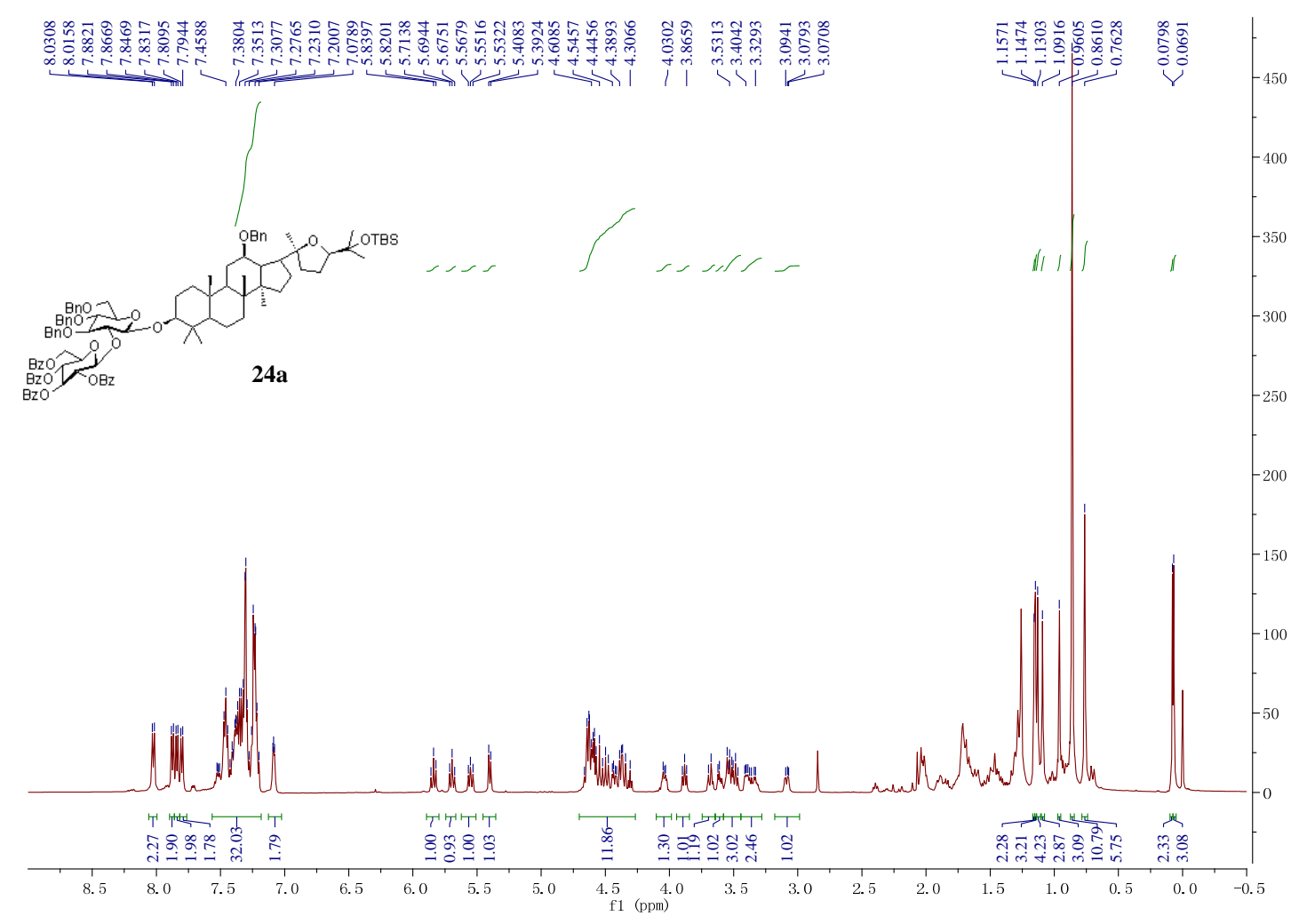

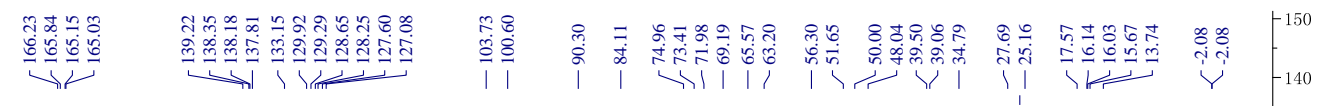

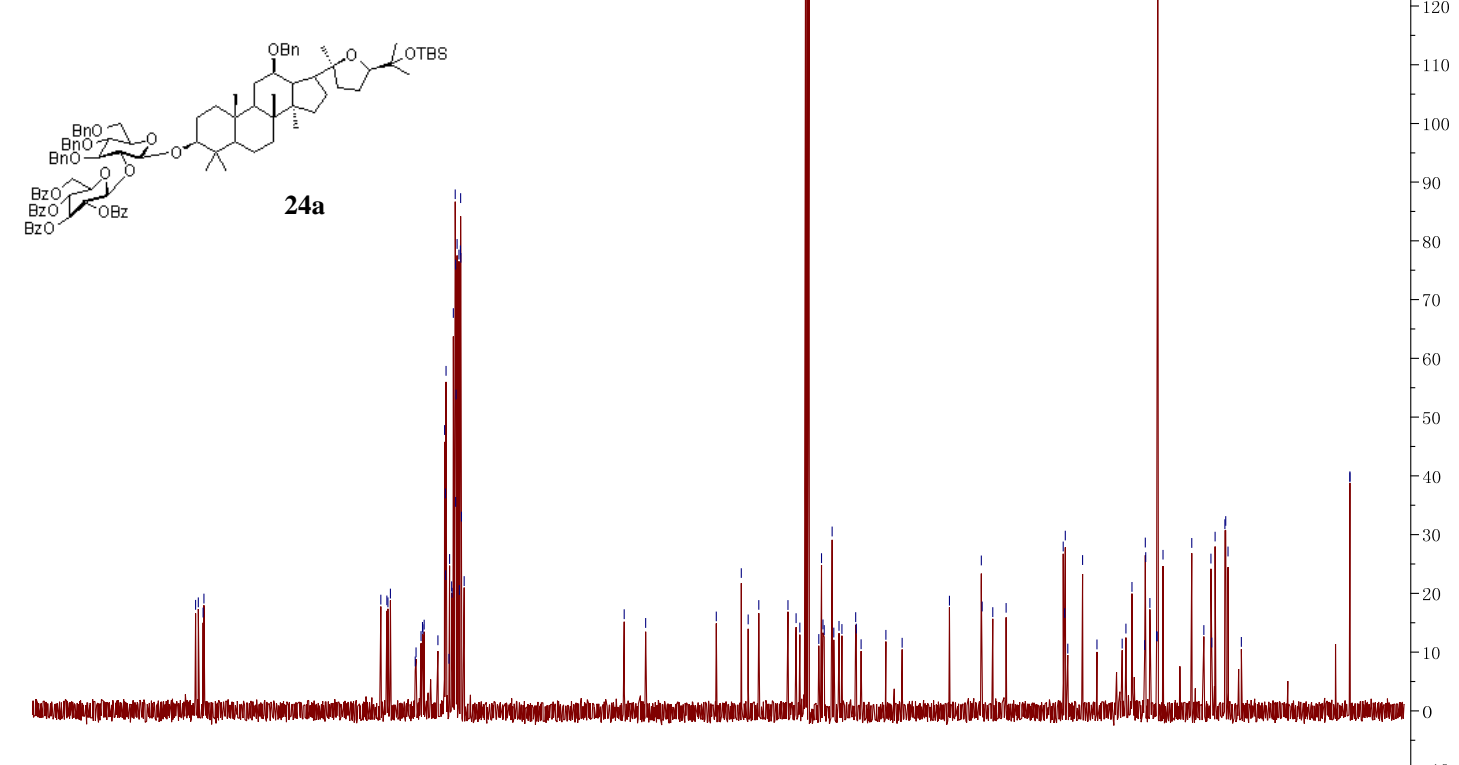

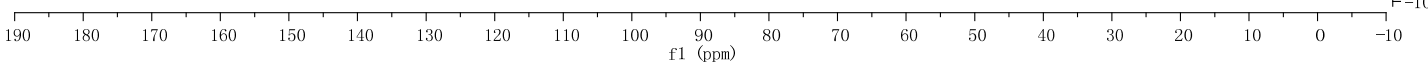




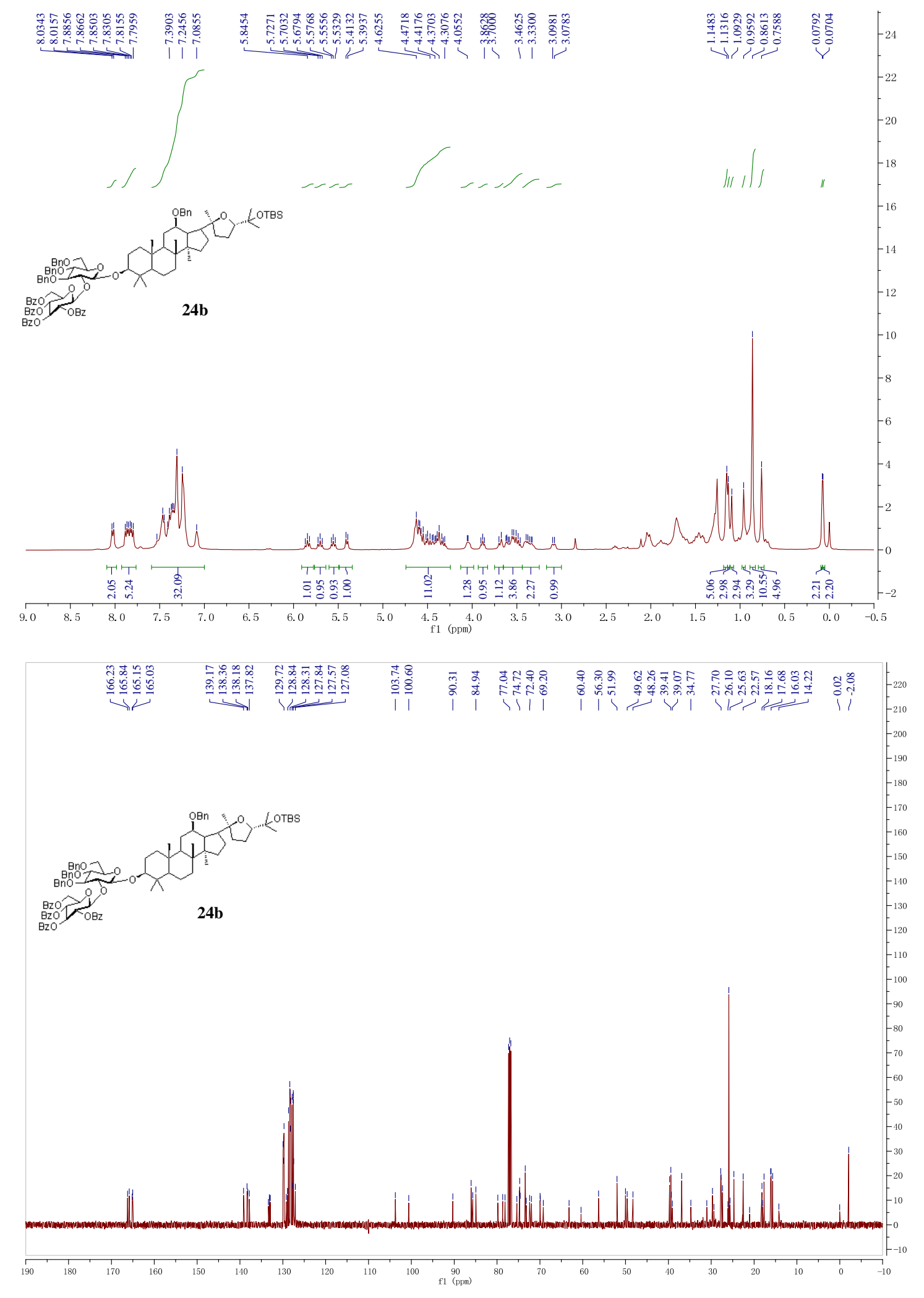




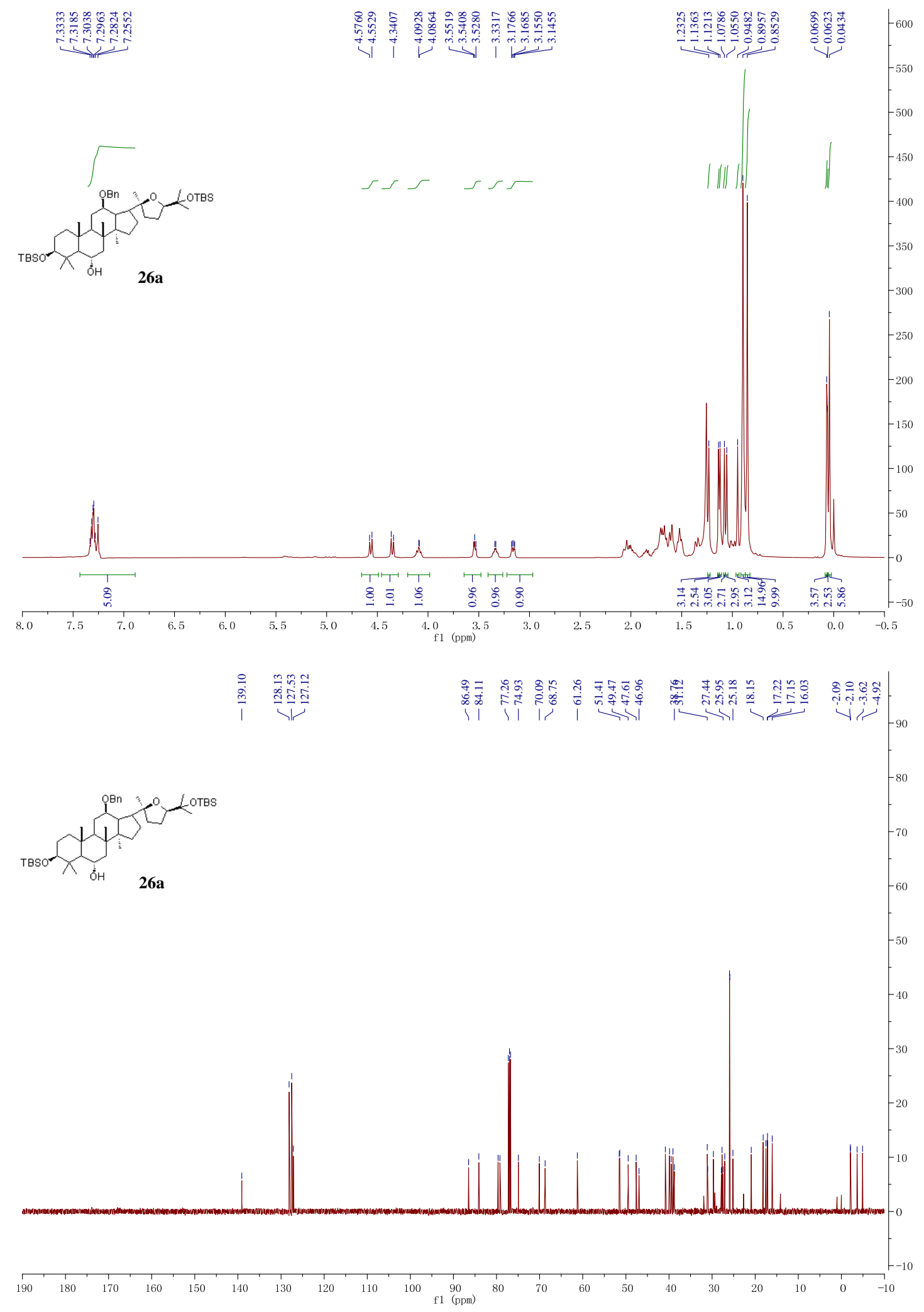




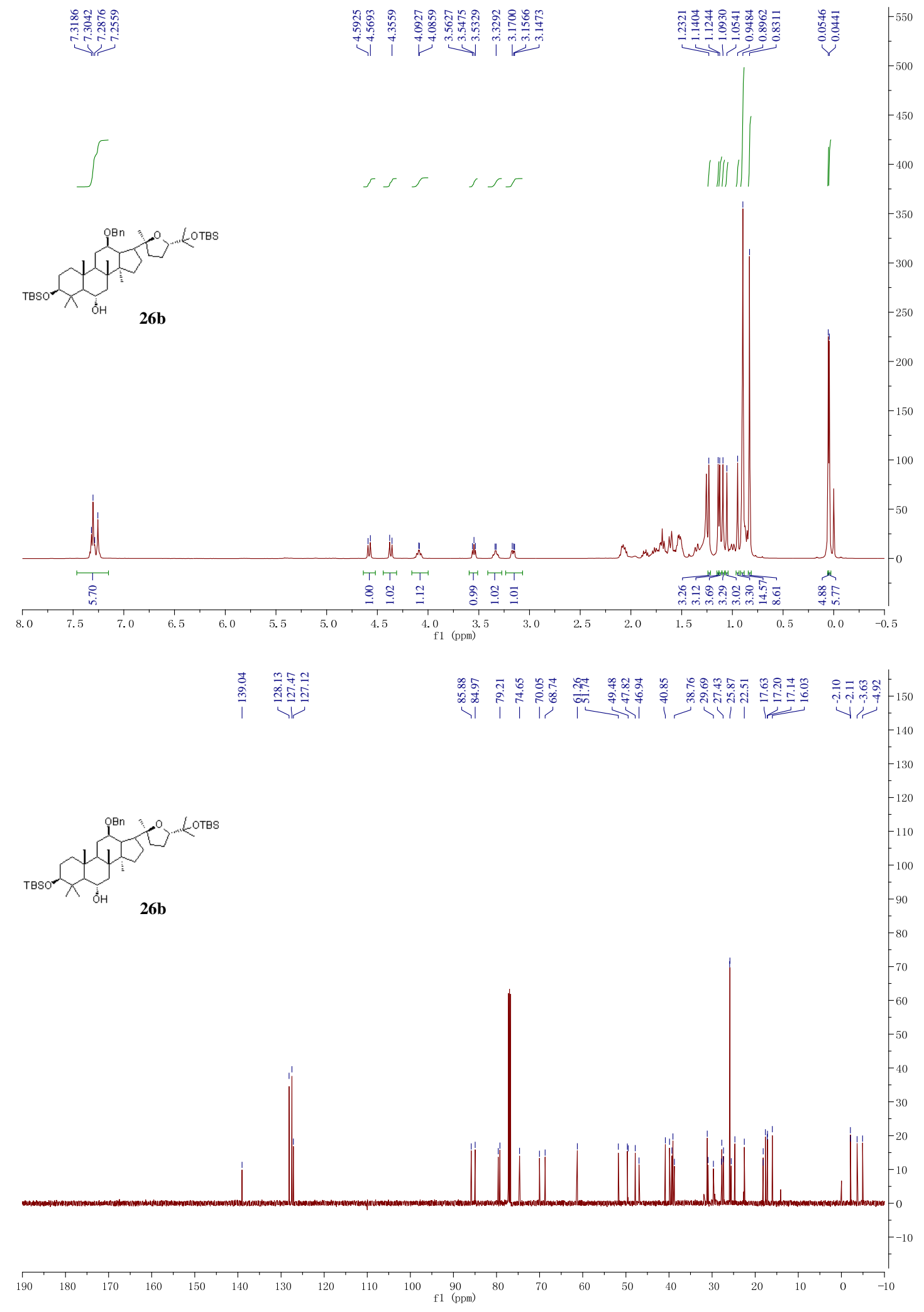



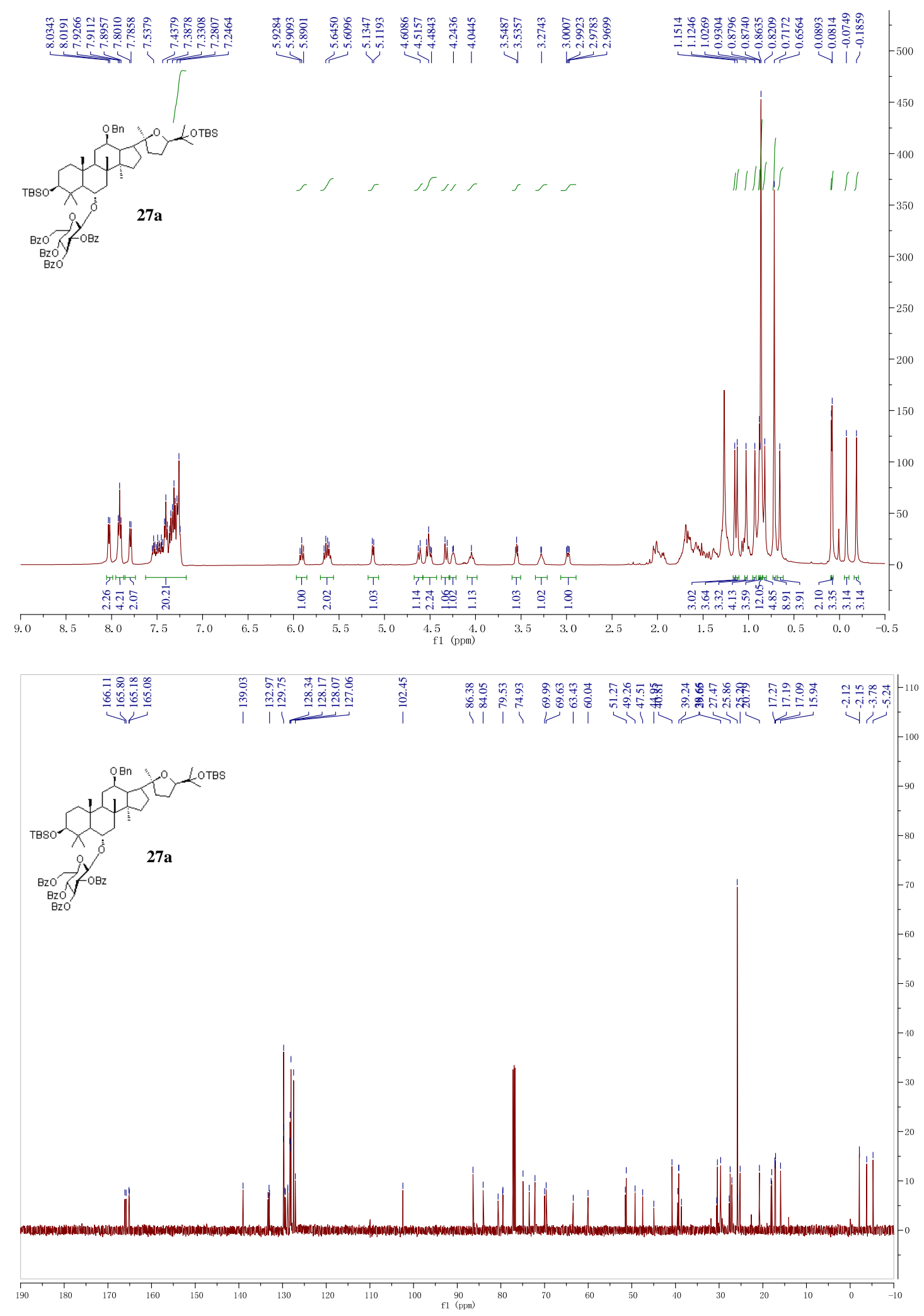


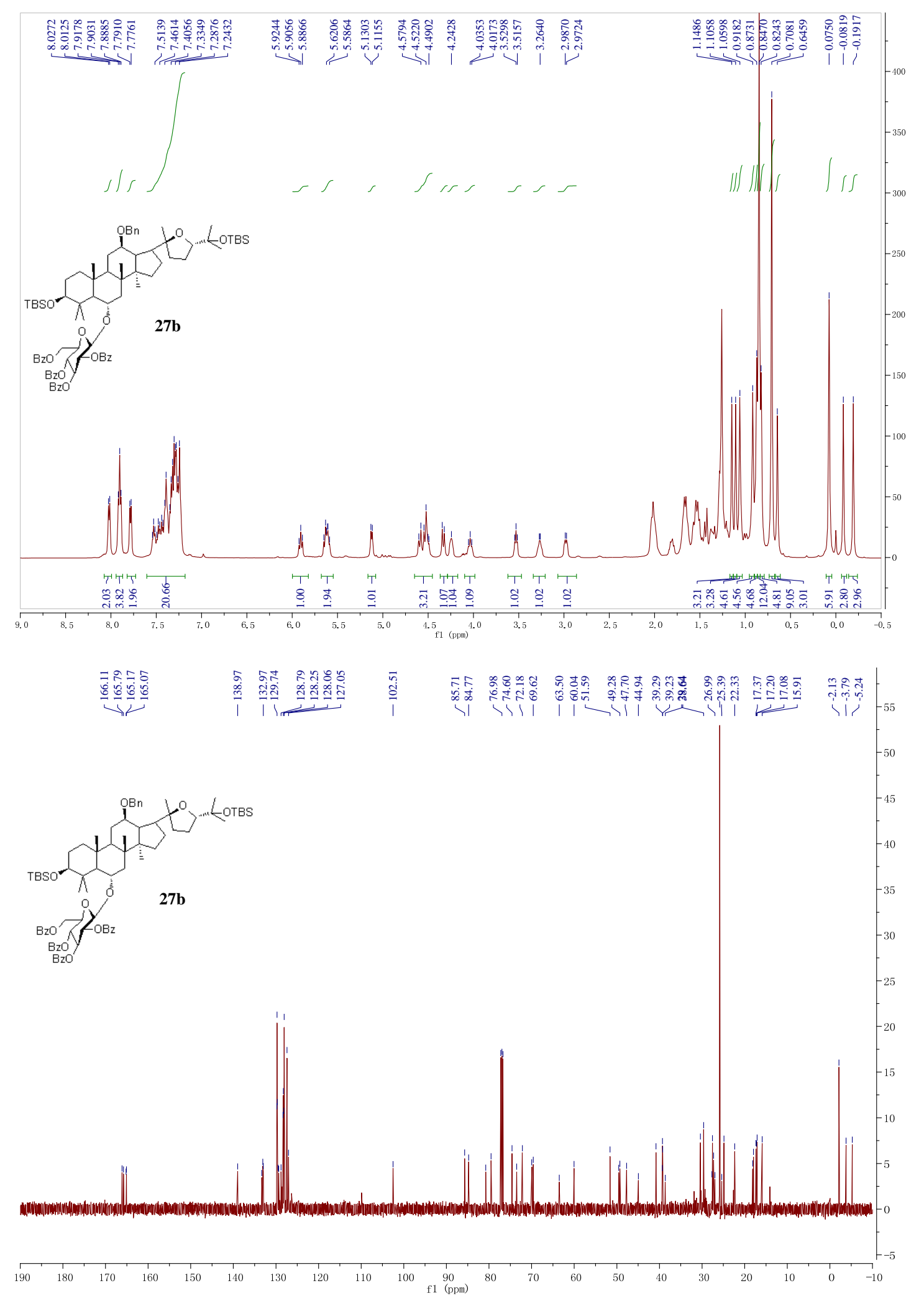



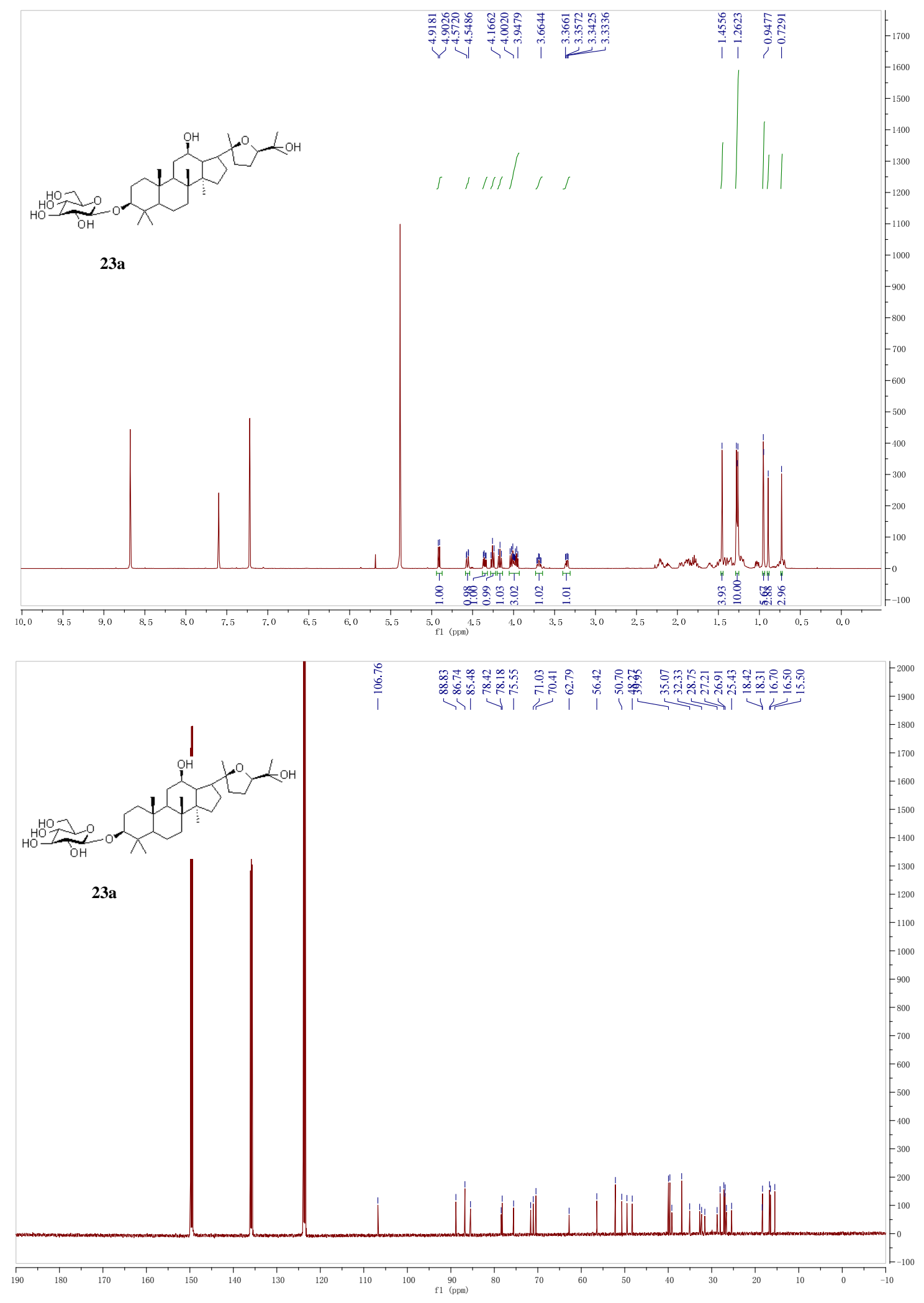

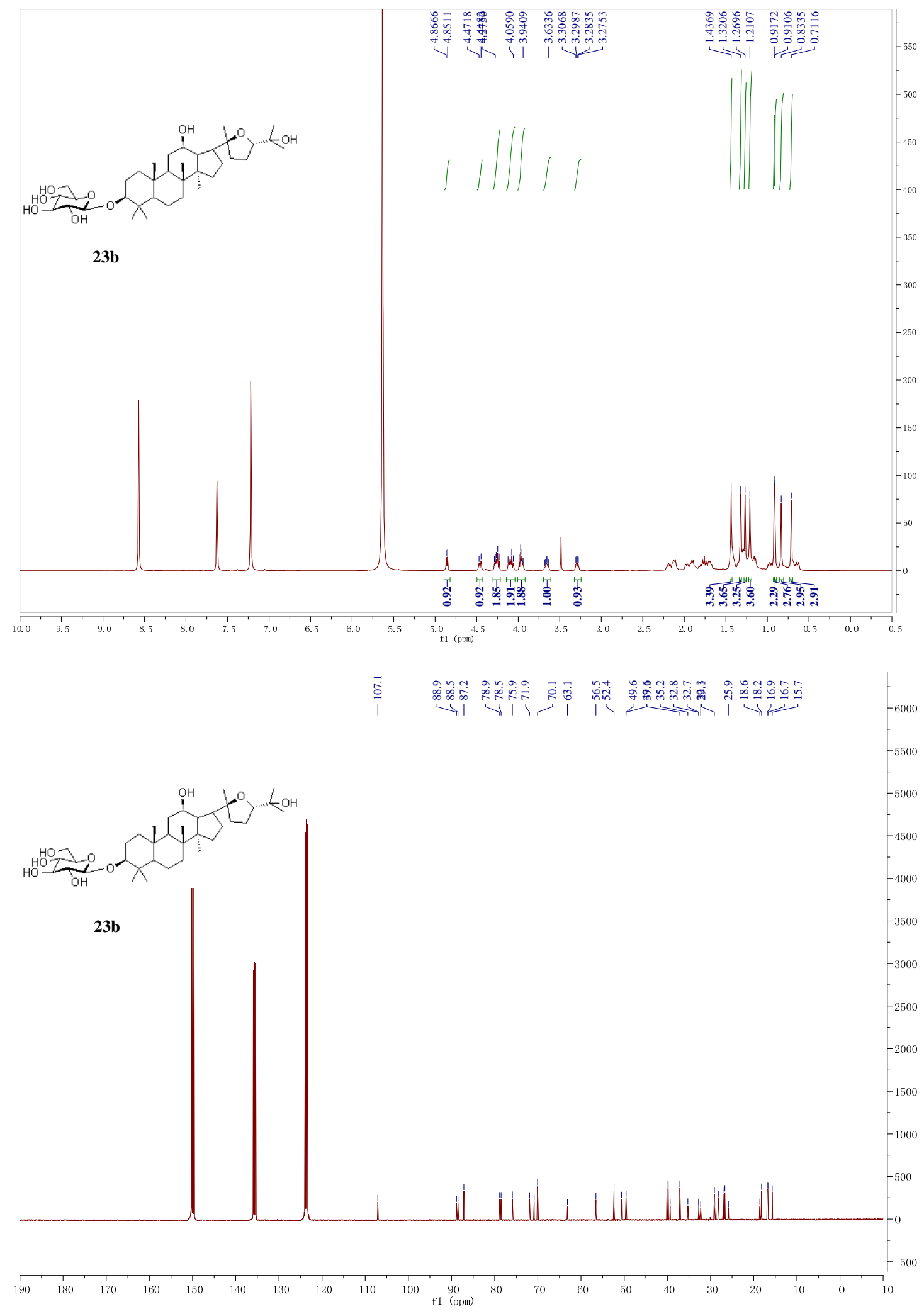

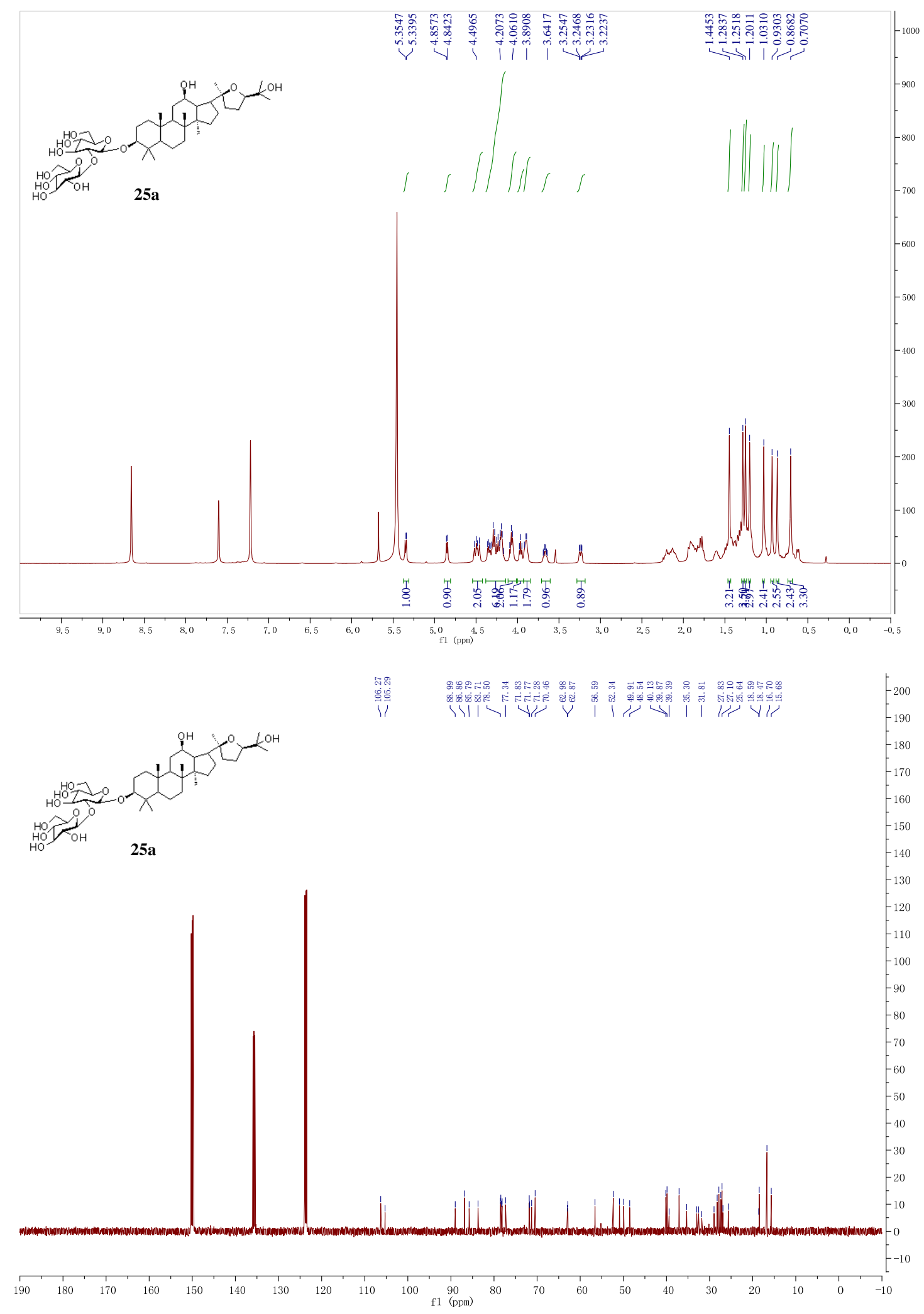


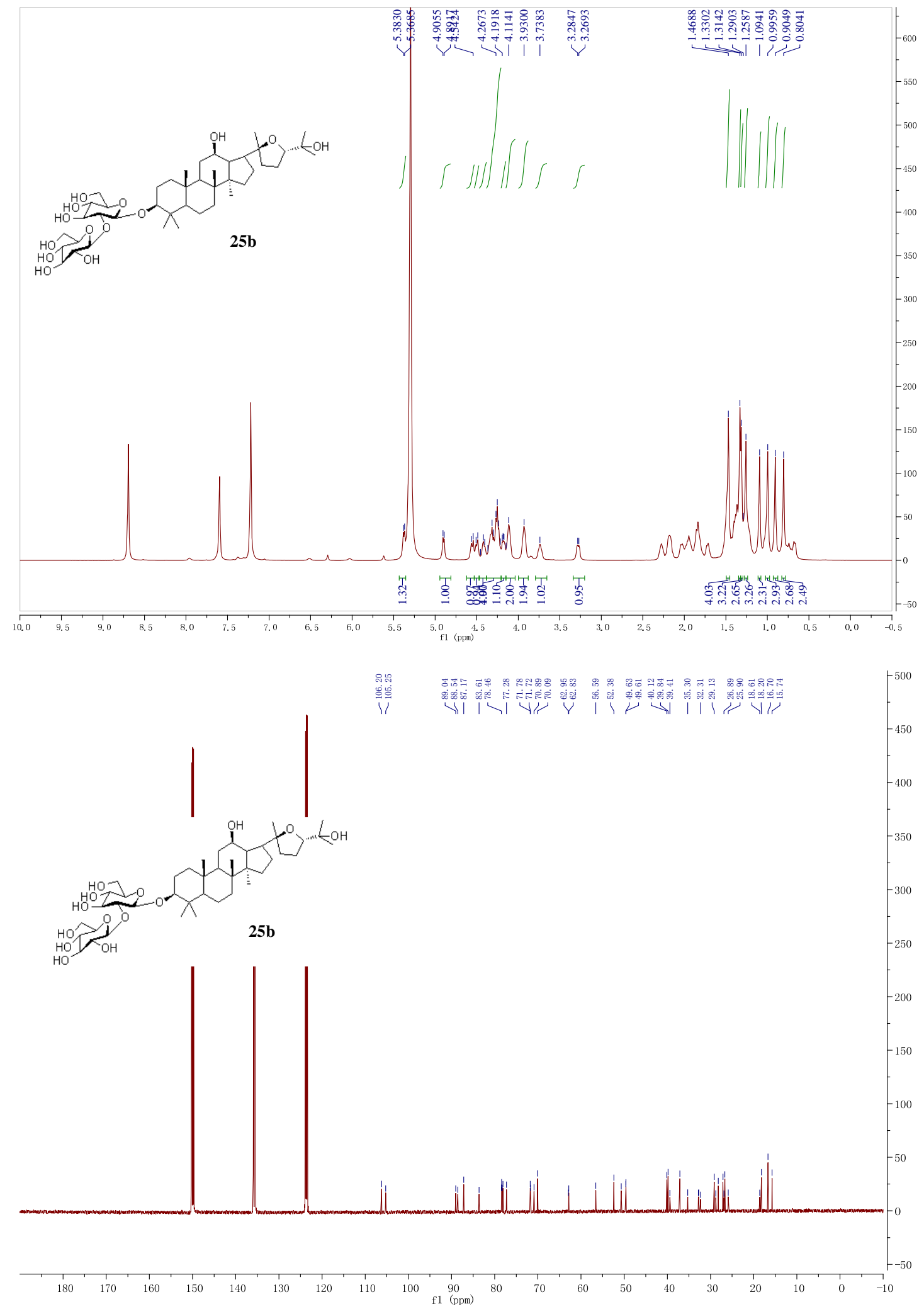



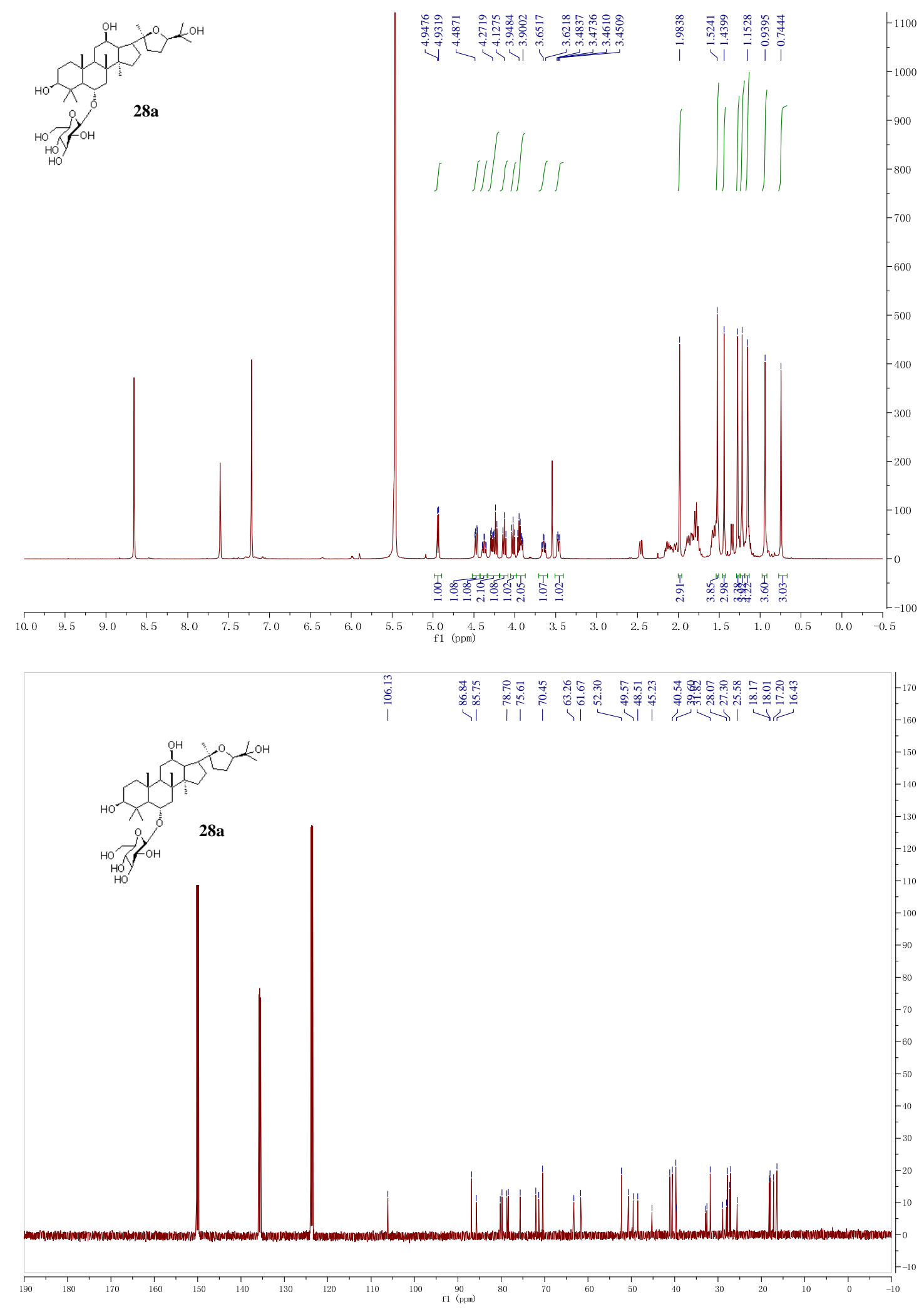

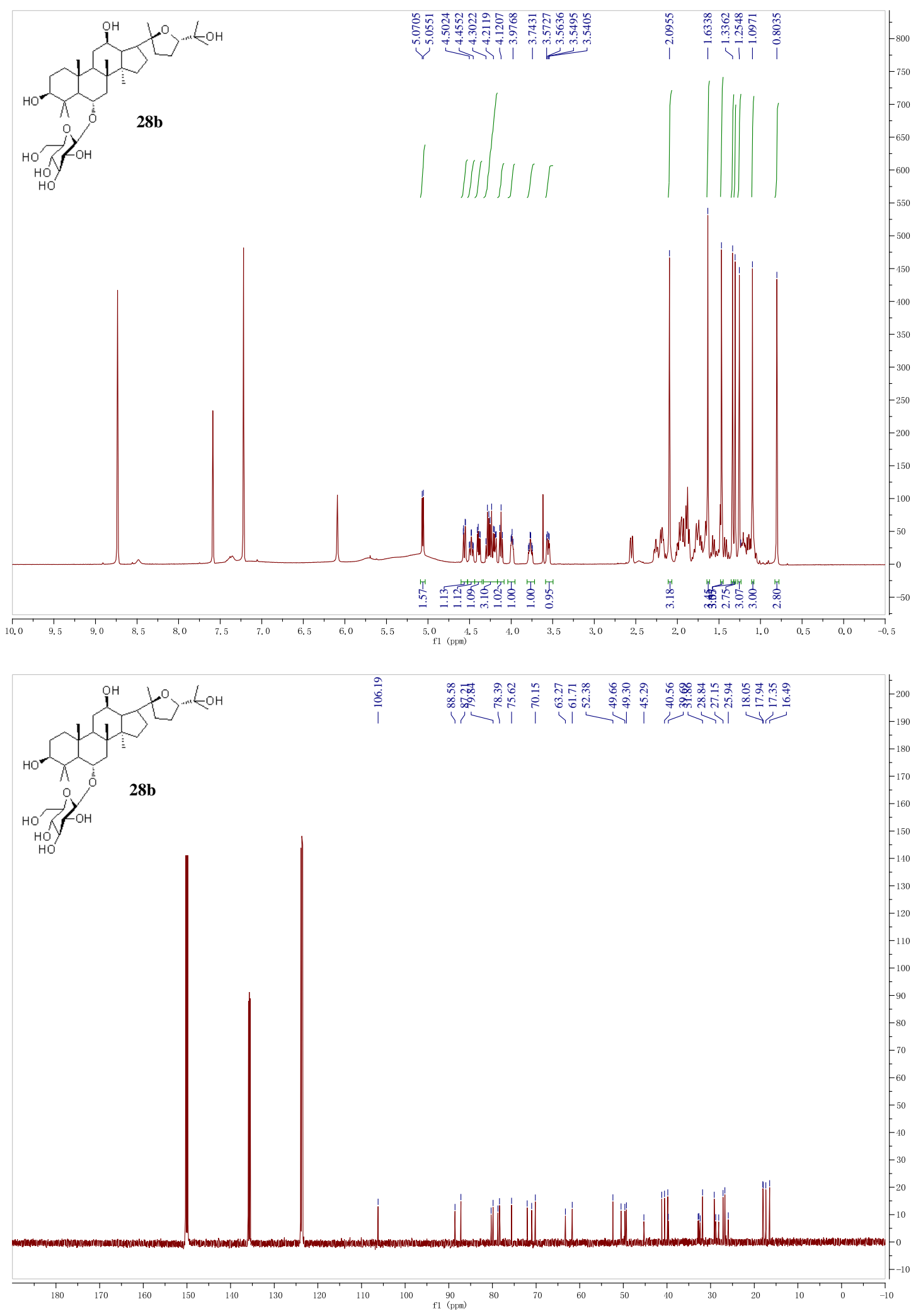

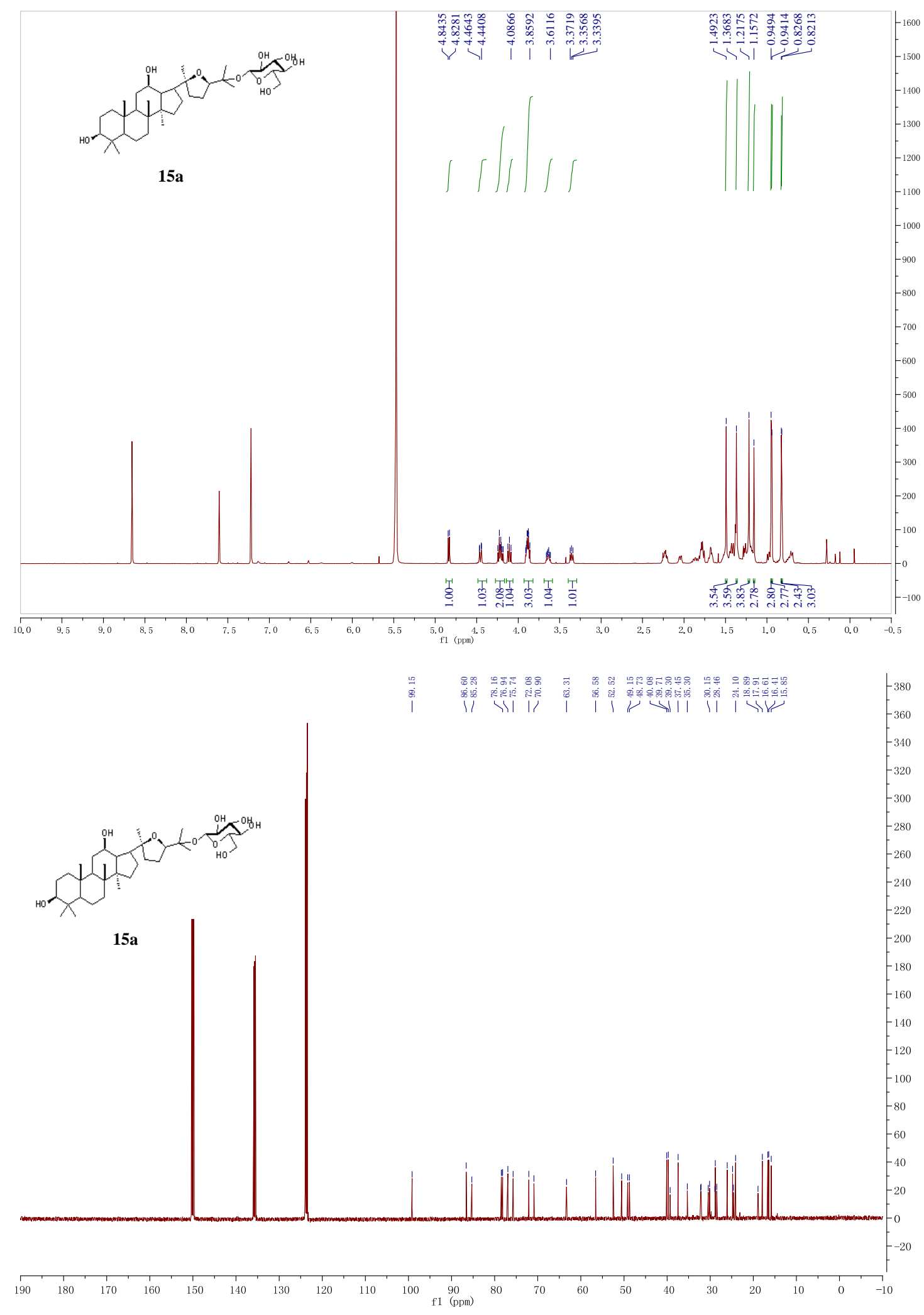

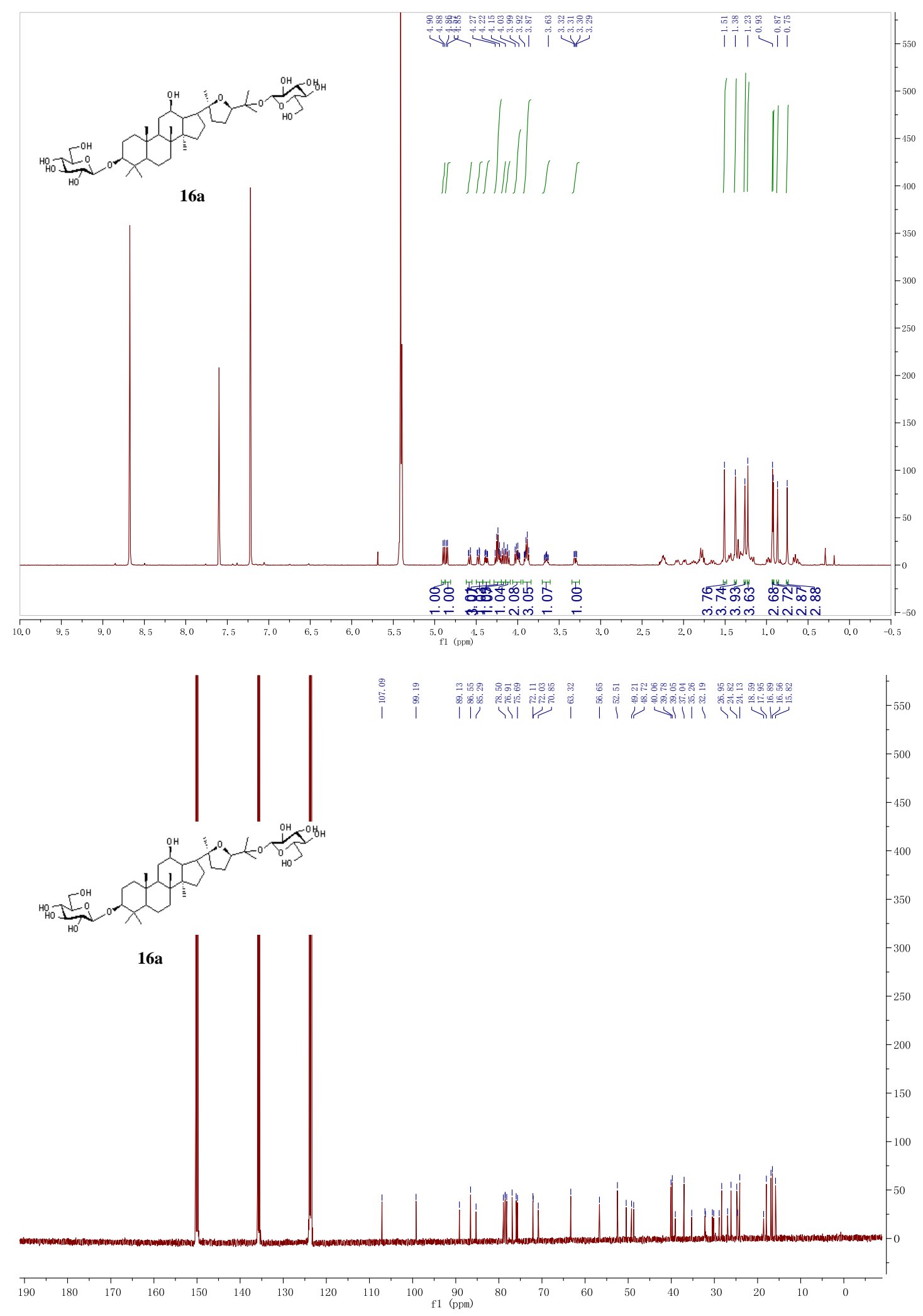


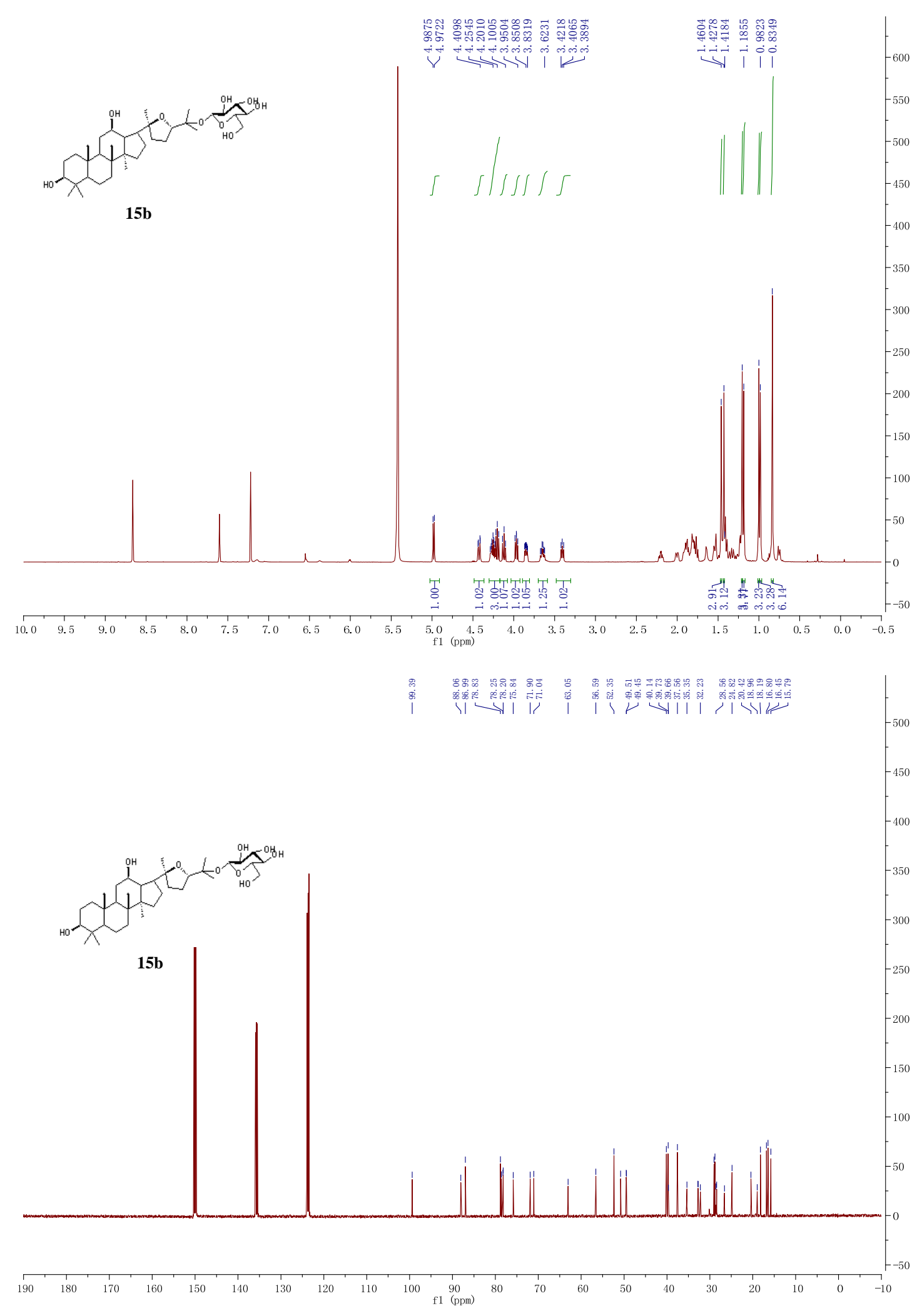



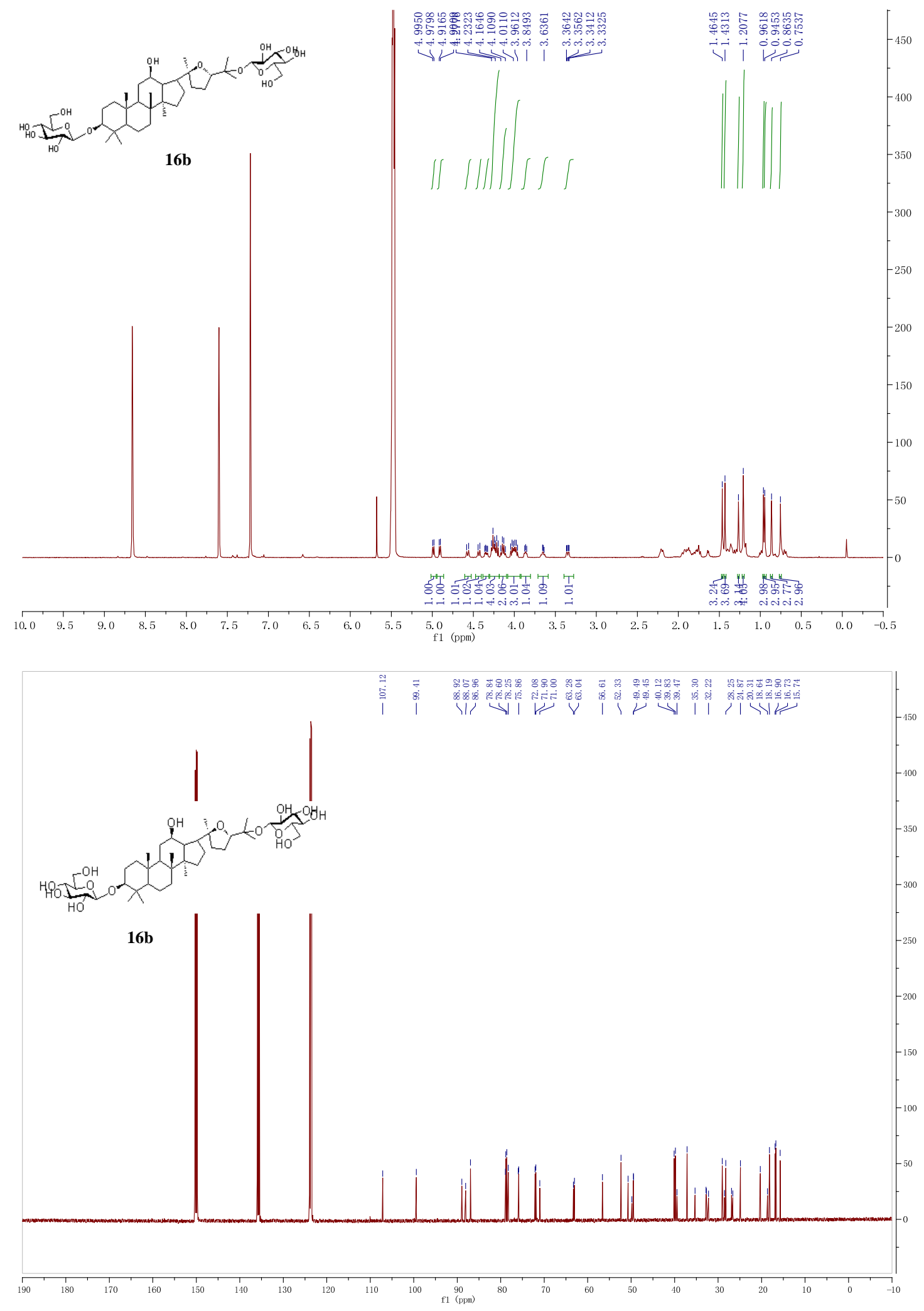

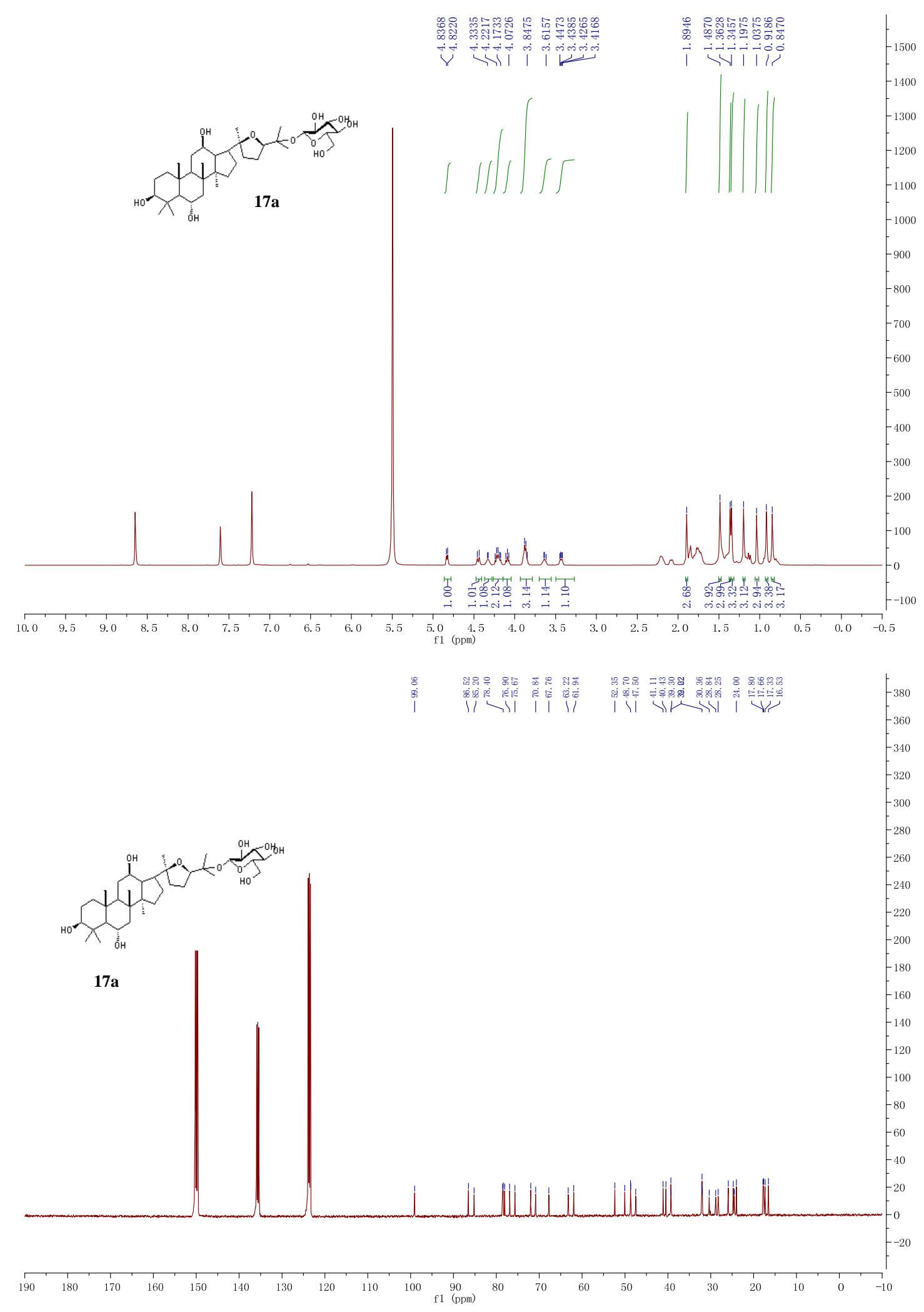

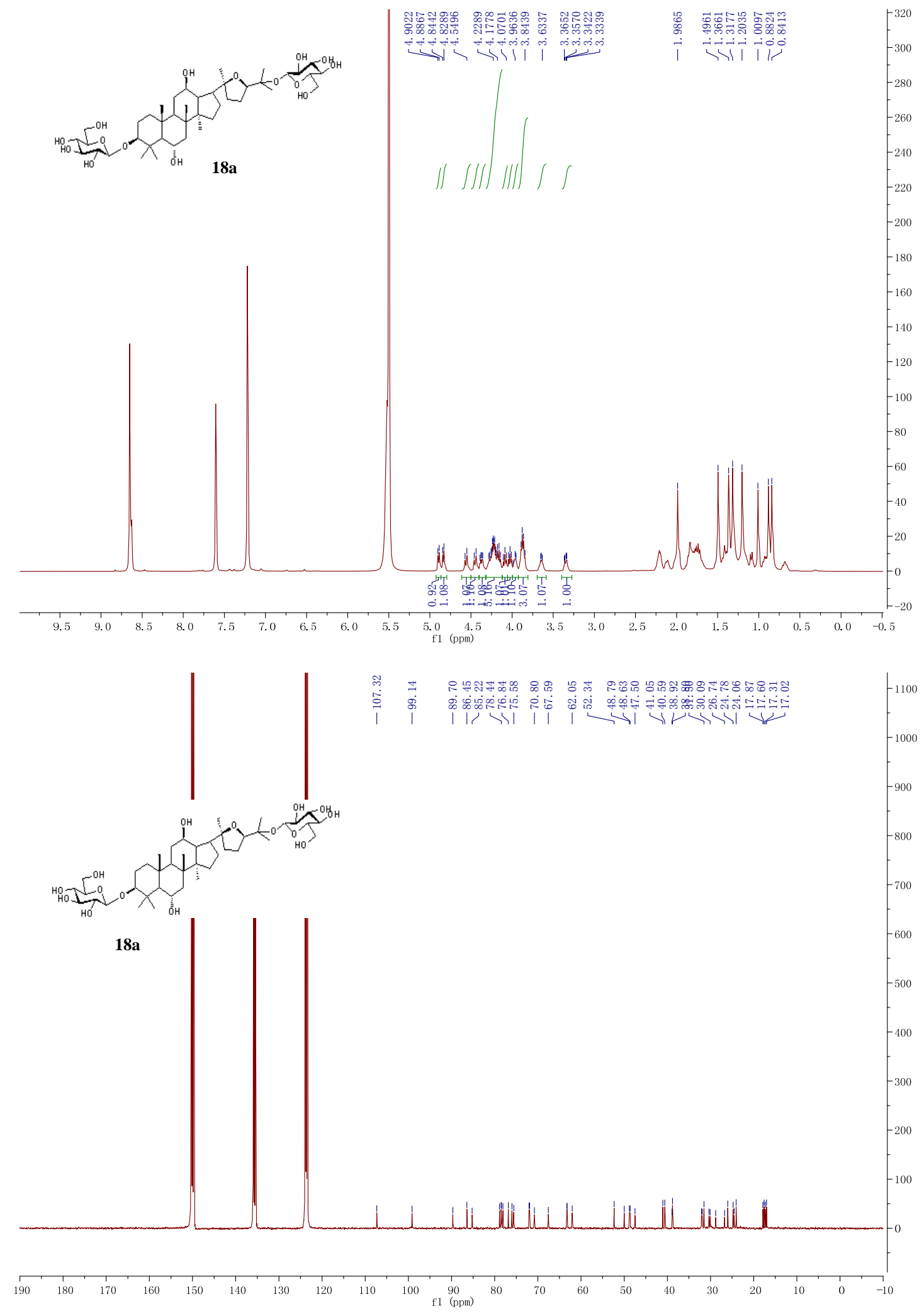

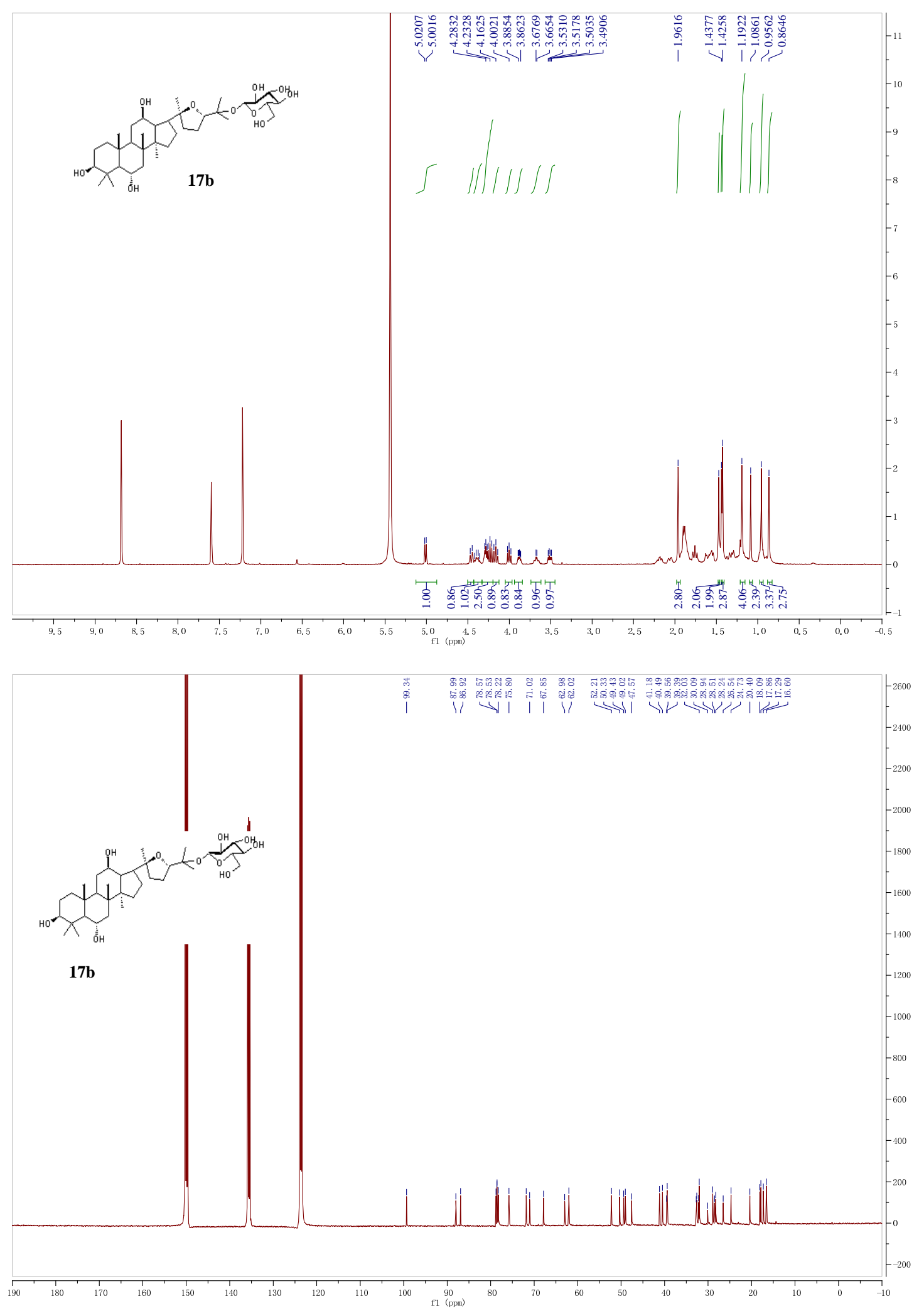

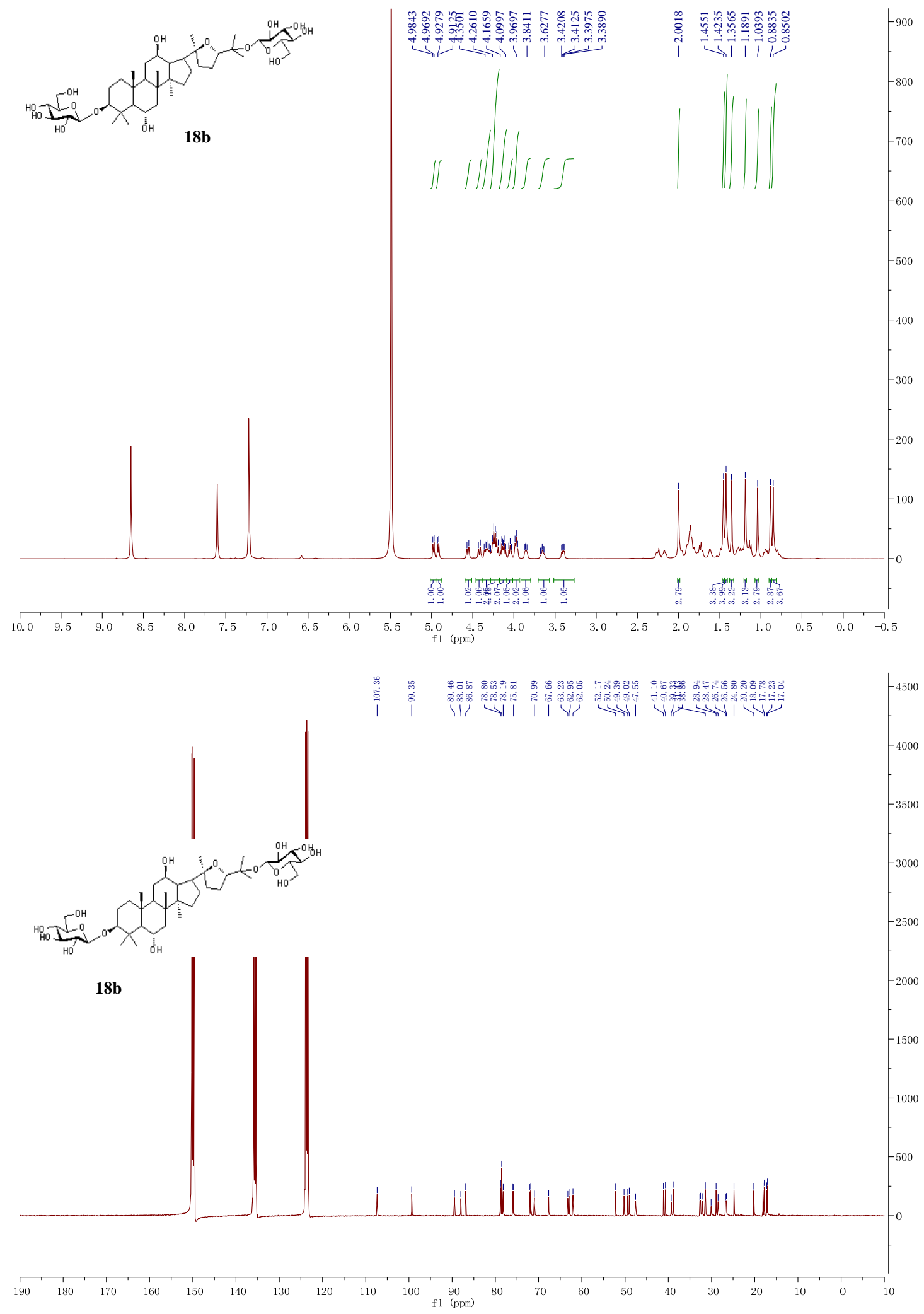


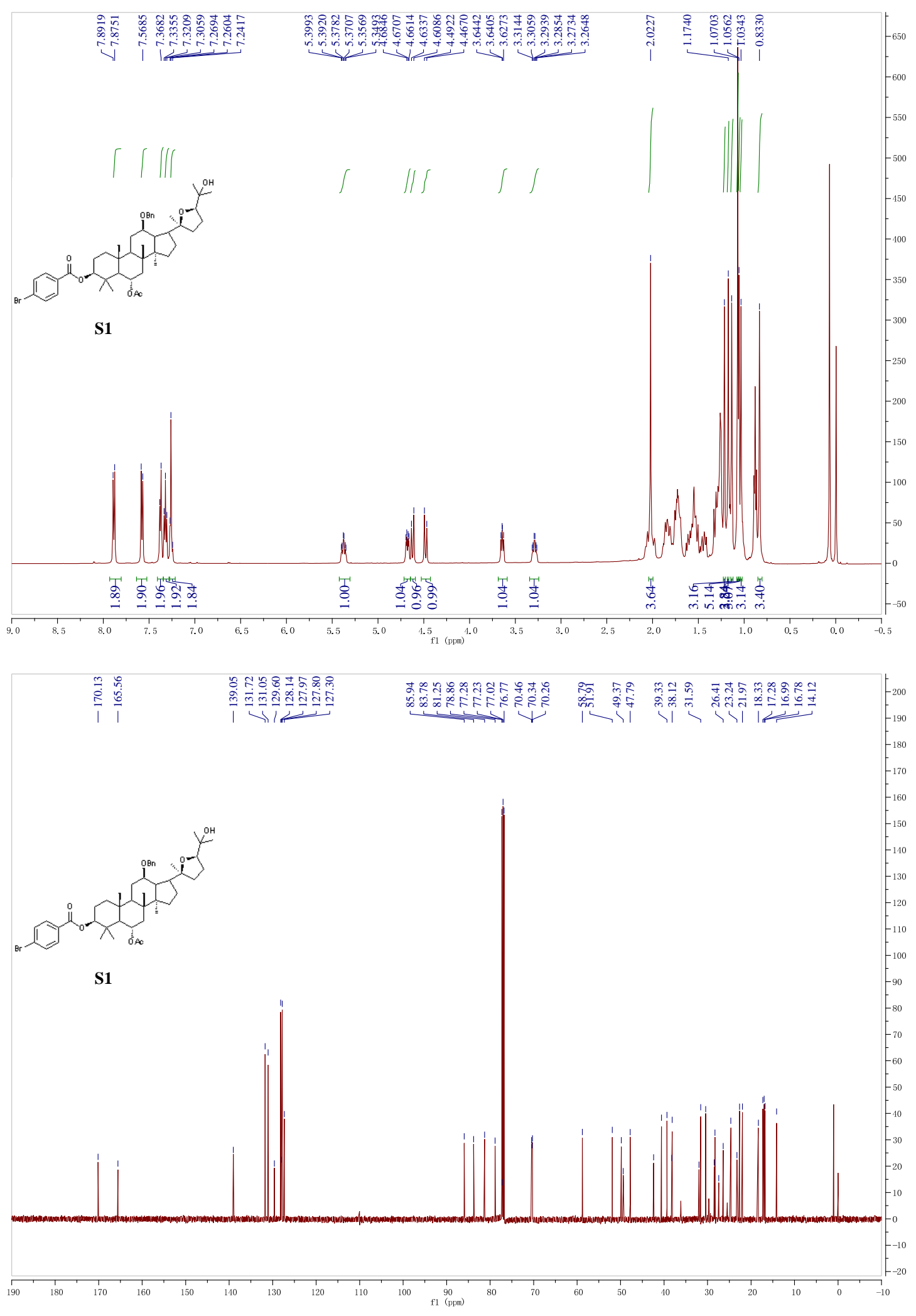

